DOE/XX/XXXXX-X

\title{
HIGH RESOLUTION/HIGH FIDELITY \\ SEISMIC IMAGING AND PARAMETER ESTIMATION FOR \\ GEOLOGICAL STRUCTURE AND \\ MATERIAL CHARACTERIZATION
}

Final Report for the Period April 15, 2001 - April 14, 2004

\author{
Ru-Shan Wu, Xiao-Bi Xie and Thorne Lay \\ UNIVERSITY OF CALIFORNIA AT SANTA CRUZ \\ Santa Cruz, CA 95064
}

Submitted June 6, 2005

PREPARED FOR THE UNITED STATES
DEPARTMENT OF ENERGY
OFFICE OF BASIC ENERGY SCIENCES

Work Performed Under Contract No. DE-FG03-01ER15144 


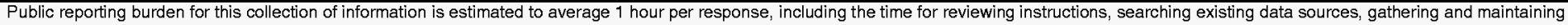

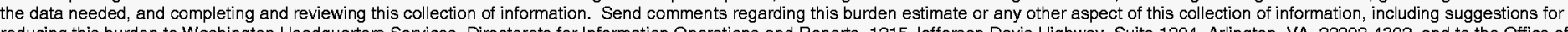

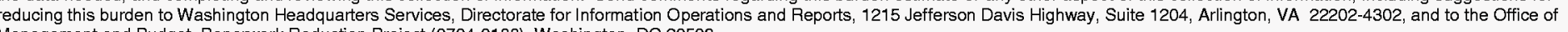
Management and Budget, Paperwork Reduction Project (0704-0188), Washington, DC 20503

\begin{tabular}{l|l}
\hline 1. AGENCY USE ONLY (Leave & 2. REPORT DATE
\end{tabular}

blank) 06/06/2005

3. REPORT TYPE AND DATES COVERED

4. TITLE AND SUBTITLE Final, $4 / 15 / 2001$ to $4 / 14 / 2004$

High Resolution/High Fidelity Seismic Imaging and Parameter

Estimation for Geological Structure and Material Characterization 5. FUNDING NUMBERS

DE-FG03-01ER15144

\section{AUTHOR(S)}

Ru-Shan Wu, Xiao-Bi Xie, and Thorne Lay

\section{PERFORMING ORGANIZATION NAME(S) AND ADDRESS(ES)}

Institute of Geophysics and

Planetary Physics,

1156 High Street

Santa Cruz, CA 95062
University of California,

Santa Cruz
8. PERFORMING ORGANIZATION REPORT NUMBER

\section{SPONSORING / MONITORING AGENCY NAME(S) AND ADDRESS(ES)}

United States Department of

Energy, office of Basic

Energy Sciences

11. SUPPLEMENTARY NOTES

This research is sponsored by the office of Basic Energy Sciences,

U. S. Department of Energy

12a. DISTRIBUTION / AVAILABILITY STATEMENT

12b. DISTRIBUTION CODE

\section{ABSTRACT (Maximum 200 Words)}

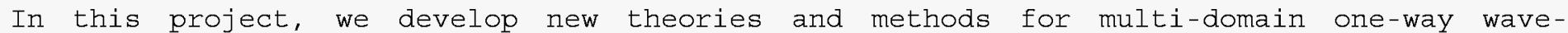

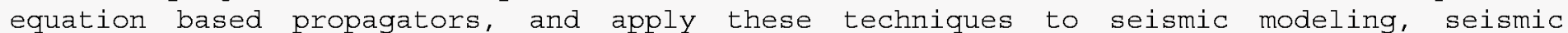

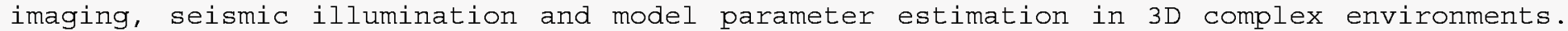

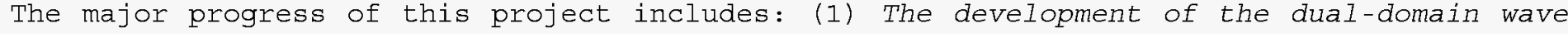

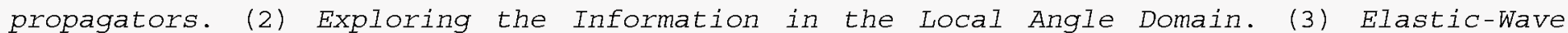
Imaging.

$\begin{aligned} & \text { 14. SUBJECT TERMS } \\
& \text { Seismic wave propagation, imaging and illumination. }\end{aligned}$
\begin{tabular}{l|l|l|}
$\begin{array}{l}\text { 17. SECURITY CLASSIFICATION } \\
\text { OF REPORT }\end{array}$ & $\begin{array}{c}\text { 18. SECURITY CLASSIFICATION } \\
\text { OF THIS PAGE }\end{array}$ & $\begin{array}{l}\text { 19. SECURITY CLASSIFICATION } \\
\text { OF ABSTRACT }\end{array}$ \\
\hline
\end{tabular}

10. SPONSORING / MONITORING AGENCY REPORT NUMBER 


\section{Table of Contents}

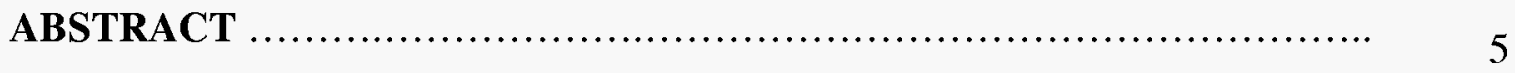

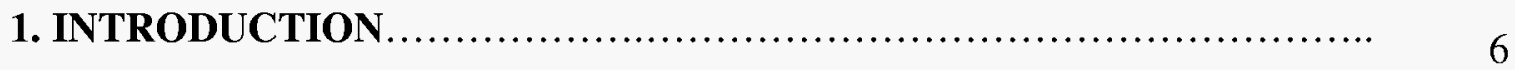

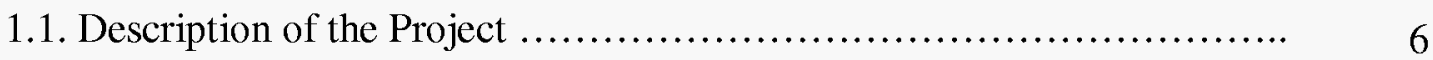

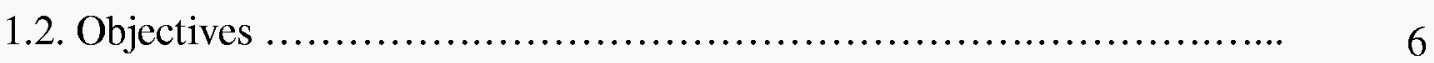

1.3. Major Progress ............................................... 6

2. ONE-WAY DUAL-DOMAIN PROPAGATOR FOR SCALAR qP-WAVE IN VTI MEDIA

2.1. The Qp-Wave Dispersion Relationship in 2-D VTI Media................ 8

2.2. Error Analysis ................................................ 10

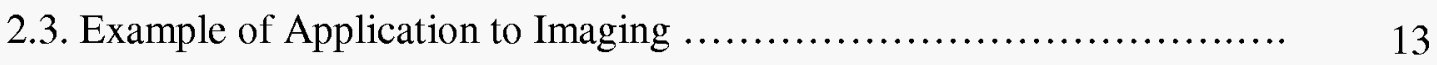

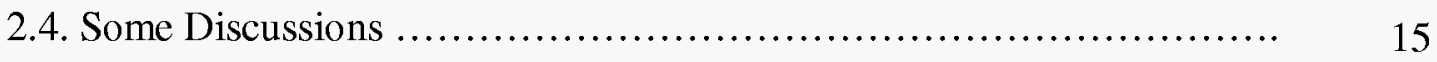

\section{IMPROVEMENT TO ELASTIC GENERALIZED SCREEN}

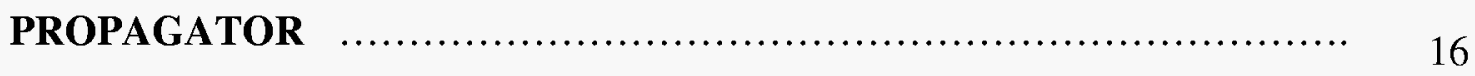

3.1. Dual-Domain Thin-Slab Formulation ........................... 16

3.2. Numerical Tests for the Elastic Thin-Slab Method .................... 20

3.3. Generalized Screen Propagator with Intrinsic Attenuation .............. 23

4. APPLICATIONS OF FAST ELASTIC-WAVE MODELING ….......... 27

4.1. AVO Modeling/Analysis ......................................... 27

4.2. Random Media Characterization and AVO Analysis ..................... 27

4.3. Frequency-Dependent Reflectivity ............................... 29

\section{EXTRACTING LOCAL ANGLE DOMAIN INFORMATION FROM}

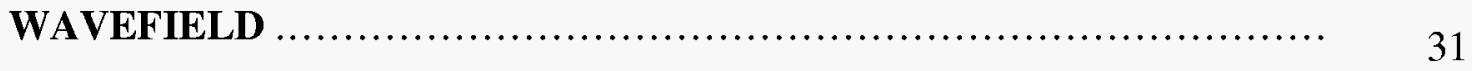

5.1. Angle Domain Analysis for Migrated Wavefields ................... 31

5.2. Numerical Examples ....................................... 34

\section{ILLUMINATION ANALYSIS IN THE LOCAL ANGLE-} DOMAIN

6.1. Angle Domain Illumination analysis and dip-response analysis........... 39

6.2. Numerical Examples of Illumination Calculation ..................... 41

6.3. Acquisition Dip Response Analysis 47 


\section{MULTICOMPONENT PRESTACK DEPTH MIGRATION USING}

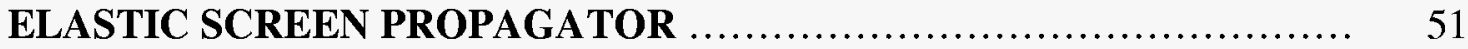

7.1. Screen Propagator for Multicomponent Elastic Waves................... 52

7.2. Wide-Angle Correction for Elastic Propagators ..................... 55

7.3. Imaging Conditions ............................................ 56

7.4. Numerical Examples .......................................... 58

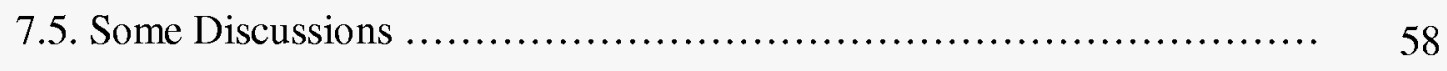

\section{CONVERTED WAVE PATH (C-PATH) IMAGING FOR STEEP}

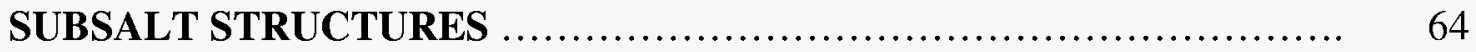

8.1. Properties of the Converted Waves .................................. 64

8.2. Forward Modeling for Illumination of Subsalt reflectors ................. 67

8.3. Acoustic Migration with Converted Wave Paths ....................... 69

9. REFERECES …............................................................ 72

10. RELATED PUBLICATIONS _.......................................... 78 


\begin{abstract}
In this project, we develop new theories and methods for multi-domain one-way waveequation based propagators, and apply these techniques to seismic modeling, seismic imaging, seismic illumination and model parameter estimation in 3D complex environments.

The major progress of this project includes:

(1) The development of the dual-domain wave propagators. We continue to improve the one-way wave-equation based propagators. Our target is making propagators capable of handling more realistic velocity models. A wide-angle propagator for transversely isotropic media with vertically symmetric axis (VTI) has been developed for P-wave modeling and imaging. The resulting propagator is accurate for large velocity perturbations and wide propagation angles. The thin-slab propagator for one-way elasticwave propagation is further improved. With the introduction of complex velocities, the quality factors Qp and Qs have been incorporated into the thin-slab propagator. The resulting viscoelastic thin-slab propagator can handle elastic-wave propagation in models with intrinsic attenuations. We apply this method to complex models for AVO modeling, random media characterization and frequency-dependent reflectivity simulation.
\end{abstract}

(2) Exploring the Information in the Local Angle Domain. Traditionally, the local angle information can only be extracted using the ray-based method. We develop a waveequation based technique to process the local angle domain information. The approach can avoid the singularity problem usually linked to the high-frequency asymptotic method. We successfully apply this technique to seismic illumination and the resulting method provides a practical tool for three-dimensional full-volume illumination analysis in complex structures. The directional illumination also provides information for angledomain imaging corrections.

(3) Elastic-Wave Imaging. We develop a multicomponent elastic migration method. The application of the multicomponent one-way elastic propagator and the wide-angle correction preserve more dynamic information carried by the elastic waves. The vector imaging condition solves the polarization problem of converted wave imaging. Both P-P and $\mathrm{P}-\mathrm{S}$ images can be calculated. We also use converted waves to improve the image of steep sub-salt structures. The synthetic data for the SEG/EAGE salt model are migrated with a generalized screen algorithm and for the converted PSS-wave path. All the sub-salt faults are properly imaged. 


\section{INTRODUCTION}

\subsection{Description of the Project}

This project is supported by the United States Department of Energy, Office of Basic Energy Sciences, and is a collaborative effort between the University of California at Santa Cruz and the Los Alamos National Laboratory. The research at UCSC is implemented at the Modeling and Imaging Laboratory, Institute of Geophysics and Planetary Physics. This report covers the research conducted at the UCSC during the project period.

\subsection{Objectives}

The purpose of this project is developing new theories and methods for multi-domain one-way wave-equation based propagators, and applying these techniques to seismic modeling, seismic migration/imaging, seismic illumination and model parameter estimation in 3D complex environments. This efficient wave-equation based method can provide excellent imaging results, and high-fidelity local parameter estimation compared with the traditional ray based methods. The resulting techniques can be used for highresolution and high-fidelity subsurface imaging; local parameter estimation of reservoir structures, such as reflection amplitude vs. angle information (AVA or AVO) and local reflection analysis.

\subsection{Major Progress}

\subsubsection{New Developments of the Dual-domain Propagators}

We continually improve the one-way wave-equation based propagators. Our goal is making the propagator capable of handling more realistic velocity models. In Chapter 2, a wide-angle propagator in transversely isotropic media with vertically symmetric axis (VTI) is developed for P-wave modeling and imaging (Han and Wu, 2003, 2005). The propagator includes the phase shift term, phase screen term, and two wide-angle terms. The resulting propagator is accurate for large velocity perturbations and wide propagation angles. Both the background medium and the perturbations can be purely isotropic, elliptical anisotropic or general VTI media. In Chapter 3, the thin-slab propagator for one-way elastic wave propagation is further improved ( $\mathrm{Wu}$ and $\mathrm{Wu}, 2003 \mathrm{a}$ ). With the introduction of complex velocities, the quality factors Qp and Qs have been incorporated into the thin-slab propagator. The resulted viscoelastic thin-slab propagator can be used to handle elastic wave propagation in models with intrinsic attenuations. In Chapter 4, we apply this propagator to the AVO modeling ( $\mathrm{Wu}$ and $\mathrm{Wu}, 2003 \mathrm{~b}, 2004)$, random media characterization and frequency-dependent reflectivity simulations (Wu and $\mathrm{Wu}, 2001$ ).

\subsubsection{Exploring the Information in the Local Angle Domain}

The second major development is exploring the local angle related information from the wavefield. Traditionally, the local angle information is only available when using the ray- 
based method. In Chapters 5, we develop wave-equation based technique to process the local angle domain information (Xie and $\mathrm{Wu}, 2002$; $\mathrm{Wu}$ and $\mathrm{Chen}, 2002$ ). The approach is based on localized plane wave analysis and can be used for almost any migration method. Using the concept of the local image matrix, useful information (local angle domain image gathers, reflector dips, etc.) can be further extracted. The local angle related information provides a whole new dimension for us to investigate the wavefield. In Chapter 6, based on local angle domain analysis, we propose a wave-equation based illumination tool (Xie et al. 2003; Wu et al., 2003). The method can avoid the singularity problem usually linked to the high frequency asymptotic method. It provides a practical tool for three-dimensional full-volume illumination analysis in complicated structures. The directional illumination also provides information for angle-domain imaging corrections.

\subsubsection{Elastic-Wave Imaging}

The third part of the report covers the progress for elastic-wave imaging. In Chapter 7, a multicomponent elastic migration method based on the elastic one-way screen propagator is developed (Xie and $\mathrm{Wu}, 2001,2005$ ). The application of the multicomponent elastic propagator and the wide-angle correction preserve more dynamic information carried by the elastic waves. The vector imaging condition solves the ambiguity of the polarization in converted wave imaging. Both P-P and P-S images can be obtained from the migration. In Chapter 8 , the converted waves are used to improve the image of steep sub-salt structures (Wu et al., 2001). Forward modeling using the finite-difference method shows that the converted wave path can penetrate the salt-body fairly efficiently while the pure P-wave is blocked due to post-critical reflections. The synthetic data for the SEG/EAGE salt model are migrated with a generalized screen algorithm and for the converted PSS-wave path. All the sub-salt faults are properly imaged. 


\section{ONE-WAY DUAL-DOMAIN PROPAGATOR FOR SCALAR qP-WAVE IN VTI MEDIA}

It is well known that isotropic algorithms applied to modeling and imaging in transversely isotropic (TI) media produce mispositioning of structures (Martin et al., 1992; Larner and Cohen, 1993; Isaac and Lawton, 1999). In response to these shortcomings many imaging methods for TI media have been developed (Meadows et al., 1987; Uren et al., 1990; Gonzalez et al., 1991; Kitchenside, 1991; Sena and Toksoz, 1993; Meadows and Abriel, 1994; Uzcategui, 1995; Le Rousseau, 1997; Le Rousseau and de Hoop, 2001; Ferguson and Margrave, 2002). Anisotropic models commonly use VTI media (Krey and Helbig, 1956; Thomsen, 1986; Schoenberg and de Hoop, 2000), and much effort has been devoted to developing one-way wave propagation methods for these media (Uren et al., 1990; Kitchenside, 1991; Meadows and Abriel, 1994; Uzcategui, 1995; Ristow and Ruhl, 1997; Le Rousseau, 1997; Le Rousseau and de Hoop, 2001; Ferguson and Margrave, 2002). For wave equation based methods, a one-way propagator is usually derived from the exact dispersion relation by use of a rational approximation. The dispersion relation in anisotropic media can be expressed as a function of phase angle and anisotropy parameters. This type of dispersion relation can be implemented in the wave-number domain. Lateral heterogeneity can be treated by use of phase-shift-plusinterpolation (PSPI) (Le Rousseau, 1997). This method requires substantial computation time, especially with anisotropic media. As an alternative, the dispersion relation for a one-way propagator can be expressed in the dual-domain (wavenumber-space domain), and successfully applied to weakly heterogeneous VTI media with a dual-domain implementation (Le Rousseau and de Hoop, 2001). Accurate one-way propagators are preferable with strongly heterogeneous VTI media. In this paper, we first derive the dispersion relation for a scalar $\mathrm{qP}$-wave using the generalized screen approximation. We then develop the one-way propagator for VTI media. The angular dependence of the propagator is analyzed using the dispersion relation and impulse responses. Finally, we demonstrate the utility of the propagator by applying it to synthetic data sets simulated using complex VTI models.

\subsection{The qP-Wave Dispersion Relationship in 2-D VTI Media}

Raytracing methods and wave equation methods are frequently used for wave propagation within anisotropic media. The raytracing methods require the use of either slowness or velocity as basic parameters. With the asymptotic expansion of the ray series for elastic wave propagation in anisotropic media, the velocities of quasi-P (qP), quasi$\mathrm{SV}(\mathrm{qSV})$ and quasi-SH (qSH) waves can be obtained from the characteristic equation of the elastic wave equation (Cerveny, 1972; Daley and Hron, 1977). For one-way wave equation methods, rational representation of the relation between the vertical and horizontal wave numbers is necessary. In this section, we give the scalar $\mathrm{qP}$-wave dispersion relation in the frequency-wavenumber domain from the full elastic wave equation for a general anisotropic medium. For detailed derivations, please see Han and $\mathrm{Wu}(2005)$. 
In order to obtain the dispersion relation of the pure qP-wave, we introduce the wave potentials to the elastic wave equation. This decouples the $\mathrm{qP}$-wave from the $\mathrm{qSV}$ - and qSH-waves. In a homogeneous VTI medium, the potential of the qSH mode is independent of the $\mathrm{qP}$ and $\mathrm{qSV}$ potentials. In an infinite homogeneous VTI solid, scalar $\mathrm{qP}-$ and $\mathrm{qSV}$-wave equations in the wavenumber domain are obtained by applying Fourier transforms. The elastic constants can be expressed in terms of the isotropic compressional velocity and the shear velocity, and the Thomsen parameters (Thomsen, 1986).

Generally, the dispersion relation has four vertical wavenumber solutions corresponding to forward and backward qP-waves and forward and backward qSV-waves. In homogeneous isotropic media, it is well known that the $\mathrm{P}$-wave and $\mathrm{S}$-wave vertical wavenumbers are independent of each other. The solutions show that in homogeneous VTI media the $\mathrm{qP}$ - and $\mathrm{qSV}$-waves are coupled. Vertical wavenumber $k_{Z}$ of the $\mathrm{qP}$ mode is a function of the $\mathrm{qSV}$ velocity, and the vertical wave number $k_{Z}$ of the $\mathrm{qSV}$ mode is a function of the $\mathrm{qP}$ velocity. Decoupled dispersion relations for the qP- and qSV-modes can be derived by mathematical methods such as rational approximation (Schoenberg and de Hoop, 2000). However, our numerical experiments show that the qSV velocity makes an insignificant contribution to the vertical wave number for the qP-mode. From the above solutions, we also note that $\mathrm{qP}$ - and $\mathrm{qSV}$-waves do not exist when the qSV-wave velocity is set equal to zero. In order to obtain the vertical wave number of the $\mathrm{qP}$-mode, we set the qSV-wave velocity equal to zero (Alkhalifah, 1998) in the dispersion relation.

An accurate one-way propagator is required for high quality seismic modeling and imaging in VTI media. As a step in the derivation of this propagator, we will apply the GSP (generalized screen propagator) approximation to the square-root operator for oneway wave propagation. We decompose the VTI medium into a homogeneous background medium and perturbation components, and decompose the vertical wavenumber into a wavenumber for the background medium and a wavenumber correction. The wavenumber correction plays a central role in improving the large angle accuracy of the wave field. By expanding the square-root of the dispersion relation using the GSP approximation (Wu, 1994; $\mathrm{Wu}, 2003$; Xie and $\mathrm{Wu}, 1998)$, the vertical wavenumbers can be derived. The sub-propagators propagate the wavefield from one thin-slab to the next. In the wavenumber domain, the propagation operation is global. For each wavenumber, the wavefield data in the thin-slab must be processed together. If the medium is heterogeneous, there will be couplings between different wavenumbers and results in low computational efficiency. This deficiency will be removed in the spatial domain. Applying the inverse Fourier transform, we represent the wavenumber correction as

$$
\Gamma_{c}(x, z)=\Gamma_{c}^{s}(x, z)+\Gamma_{c}^{f}(x, z),
$$

where $\Gamma_{c}^{s}(x, z)$ is the phase-screen correction, $\Gamma_{c}^{f}(x, z)$ is the finite-difference correction. The phase-screen correction for the phase-shifted wavefield is represented as

$$
\phi_{j+1}(x, z)=p_{c}^{s}(x, z) \phi_{j+1}^{b}(x, z)
$$

Where $p_{c}^{s}(x, z)=\exp \left[i \Gamma_{c}^{s}(x, z) \Delta z\right]$, and $p_{j+1}^{b}(x, z)=F T^{-1}\left\{\tilde{\phi}_{j+1}^{b}\left(k_{x}, z\right)\right\}$. The finitedifference correction for the phase-screen corrected wavefield is written as 


$$
\partial_{z} \phi_{j+1}(x, z)=\Gamma_{c}^{f}(x, z) \phi_{j+1}(x, z) .
$$

If perturbations exist, the propagator includes both the wavenumber and spatial domain sub-propagators; if no perturbations exist, the propagator includes only the wavenumber domain sub-propagator, which is the well-known phase-shift propagator for homogeneous VTI media (Kitchenside, 1991; Gonzalez et al., 1991; Meadows and Abriel, 1994). The spatial domain sub-propagators correct the local wavefield for local perturbations. Therefore, the localized operation works even if the thin-slab is heterogeneous, i.e., the perturbations are not equal at all locations within the thin-slab. The propagator can be used for complex VTI solids.

\subsection{Error Analysis}

In this section, the accuracy of the propagator is discussed. We first calculate the error caused by the dispersion relation approximation using different anisotropy and perturbation values in the frequency-wavenumber domain. We then calculate the impulse response using the dual-domain propagator with different background media and perturbation components, and compare it to an accurate impulse response calculated with the phase-shift method for homogeneous VTI media.

\subsubsection{Accuracy of Dispersion Relation}

To test the approximate dispersion relation for different anisotropy and velocity perturbations, we consider four VTI models as shown in Table 1. We use very strong anisotropy (the third and the forth models which might be unrealistic) to show its mathematical flexibility.

TABLE 1. Model parameters for Figure 1

\begin{tabular}{||c|c|c|c|c||}
\hline \hline & $\delta_{0}$ & $\varepsilon_{0}$ & $\alpha_{0}(\mathrm{M} / \mathrm{S})$ & PERTURBATION (\%) \\
\hline$(\mathrm{a})$ & 0.025 & 0.0005 & 2500 & 100 \\
\hline (b) & 0.100 & 0.075 & 1250 & 300 \\
\hline (c) & 0.043 & 0.114 & 714 & 600 \\
\hline (d) & 0.082 & 0.064 & 455 & 100 \\
\hline
\end{tabular}

Figure 1 shows the approximate and exact dispersion relations for a frequency of $20 \mathrm{~Hz}$. The solid curves are exact dispersion relations in VTI media, and the short-dashed curves are their corresponding approximations. In order to see the effect of anisotropy we also show the exact isotropic dispersion relations (the dotted curves). Figure 1a describes the dispersion for weak anisotropy and moderate constant perturbation (100\%). The parameters of the background medium are $\delta_{0}=0.025, \varepsilon_{0}=0.0005, \alpha_{0}=2500 \mathrm{~m} / \mathrm{s}$. The approximate dispersion relation has nearly perfect agreement with the accurate dispersion. Figure $1 \mathrm{~b}$ is the dispersion in a strong VTI medium $\left(\delta_{0}=0.100, \varepsilon_{0}=0.075\right.$, $\alpha_{0}=1250 \mathrm{~m} / \mathrm{s}$, and perturbations $300 \%$ ). This shows that the propagator has a fairly good accuracy. Figure 1c and 1d show two cases of very strong anisotropy with very 
large perturbations $\left(\delta_{0}=0.043, \varepsilon_{0}=0.114, \alpha_{0}=714 m / s \quad\right.$ and $\delta_{0}=0.082, \varepsilon_{0}=0.064, \alpha_{0}=455 \mathrm{~m} / \mathrm{s}$, and perturbations $600 \%$ and $1000 \%$ ). The approximations have a nearly perfect match with the exact dispersion relations even for strong perturbations of the anisotropy and velocity.

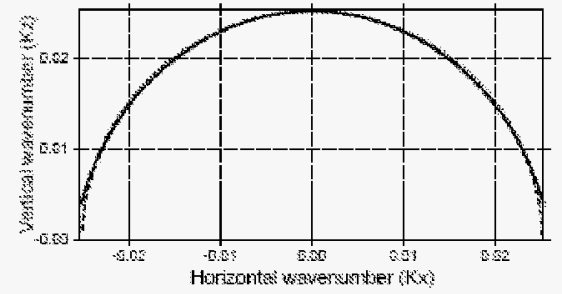

(a) Perturbation: $100 \%$

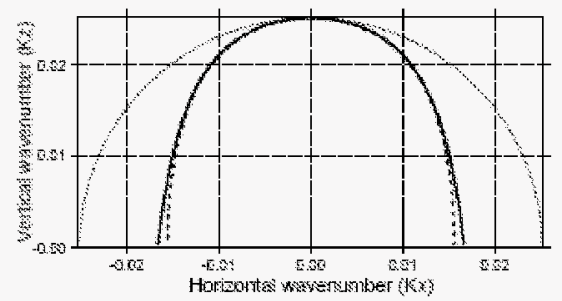

(c) Perturbation: $600 \%$

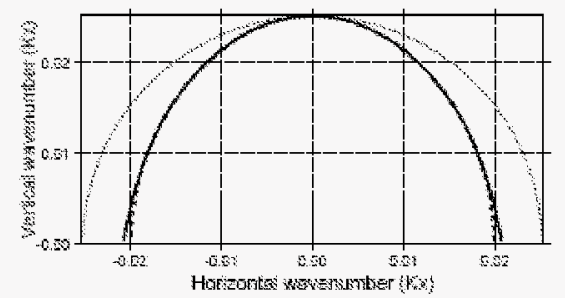

(b) Perturbation: $300 \%$

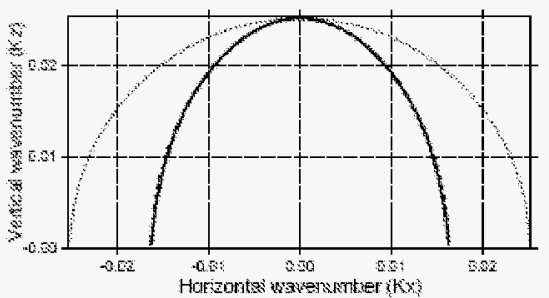

(d) perturbation: $1000 \%$

Figure 1. Approximate and exact dispersion relations for homogeneous media with different background media and perturbation values showed in Table 1. The solid curves show the exact dispersion relations, the short-dashed curves show their corresponding approximations, and the dotted curves show their corresponding exact isotropic dispersion relations.

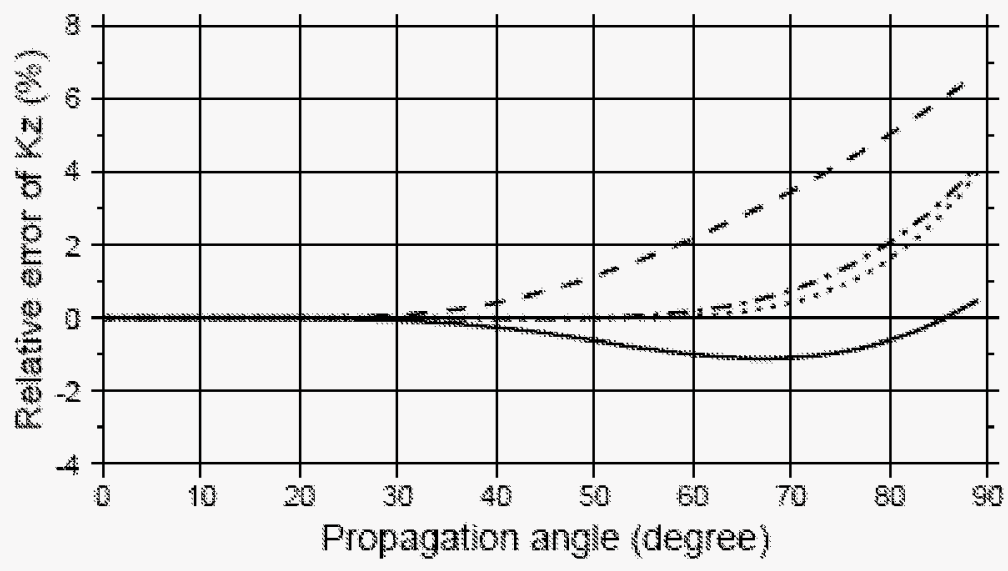

Figure 2. The relative errors of the propagator for different angles in a weakly anisotropic medium with moderate constant perturbations (the dotted curve), a strongly anisotropic medium with large constant perturbations (the dot-dashed curve), and two very strongly anisotropic media with two larger perturbation values (the dashed curve and the solid curve).

Figure 2 presents corresponding relative errors for the four cases in Figure 1 at different propagation angles. From Figure 2 we see that the dispersion relation is exact in the vertical direction which is the feature of phase-screen approximation. The error is usually less than $1 \%$ for the propagation angles less than $45^{\circ}$, and less than $10 \%$ at the largest 
propagation angle. This proves that the dispersion equation has a close approximation to the exact dispersion relation.

\subsubsection{Accuracy of the impulse response}

The propagator for wavefield modeling and imaging is implemented in the frequencywavenumber domain and in the frequency-spatial domain; the phase-shift implemented in frequency-wavenumber domain and the phase-screen and wide-angle corrections in frequency-spatial domain. The wide-angle corrections are implemented with implicit finite-difference procedures. In contrast to the computation of the dispersion relation, the implementation in the dual-domain will introduce artifacts due to the use of the finitedifference method. These artifacts are suppressed by tapering in the wavenumber domain, although the procedure will cause some distortion of amplitudes at very wide-angles.

To analyze the accuracy of the propagator, we take several tests with different perturbation values. The qP-wave velocity of the model is $5000 \mathrm{~m} / \mathrm{s}$, and $\delta=0.3, \varepsilon=0.2$. The perturbations are $100 \%, 200 \%, 300 \%, 400 \%$ respectively. Spatial samples are 280 in $\mathrm{x}$-direction and 140 in $\mathrm{z}$-direction, and $\mathrm{dx}=\mathrm{dz}=15 \mathrm{~m}$. The number of time samples is 512 and the sampling interval is $4 \mathrm{~ms}$. The peak frequency of the Ricker wavelet is $20 \mathrm{~Hz}$. Figure 3 shows corresponding responses at $300 \mathrm{~ms}$ computed with the propagator. In Figure 3, the responses with solid lines are computed with the phase-shift method in actual media, while the responses with dotted lines are computed with the approximate propagator. The propagation at large angles is tapered for the approximate propagator, while we do not apply any taper for the phase-shift propagator. As shown in the last section, in a medium without any horizontal perturbations the wave field computed with the phase-shift propagator is accurate.

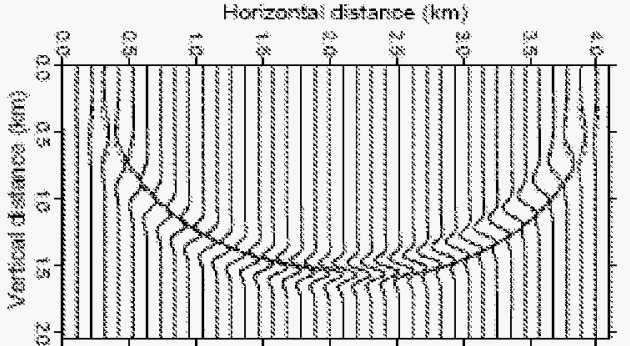

(a)

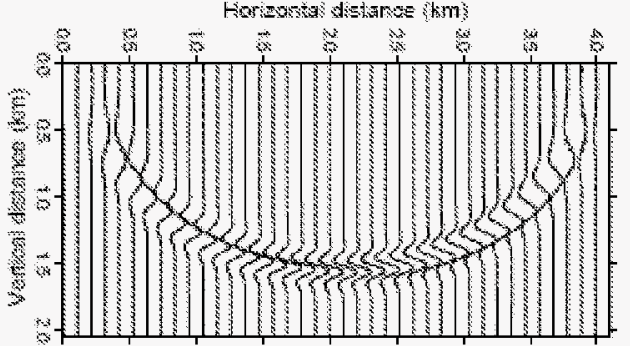

(b)

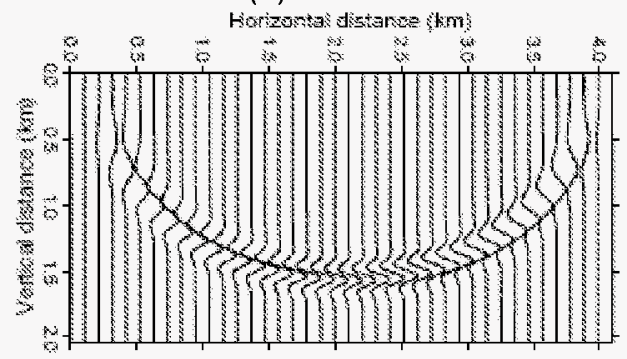

(c)

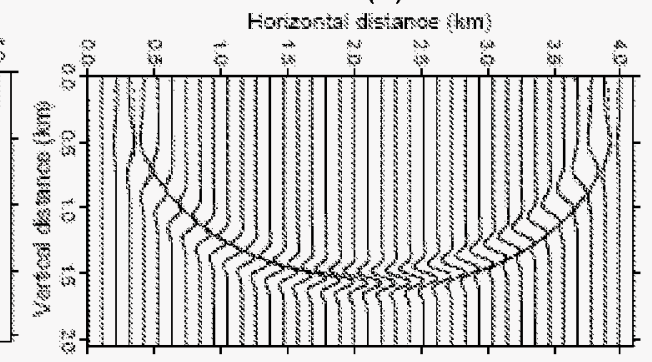

(d)

Figure 3. Responses in a homogeneous VTI medium. The responses with solid lines are computed with a phase-shift method in actual media, the responses with dotted lines are computed with the approximate propagator that propagates in a VTI background, with constant percentage perturbations of (a) $100 \%$, (b) $200 \%$, (c) $300 \%$, (d) $400 \%$. 
Figure 3 demonstrates that the results from our propagator in media with different perturbations are in close agreement with those from the phase-shift method in the true media. The propagator has a good degree of accuracy even for large perturbations and at wide propagation angles. Figure 4 describes relative errors corresponding to the approximate responses in Figure 3. The errors of the dispersion relation and those of the dual-domain propagator exhibit similar behavior. From Figure 3 we see that with increasing levels of perturbation, the relative error seems to increase slowly at large angles.

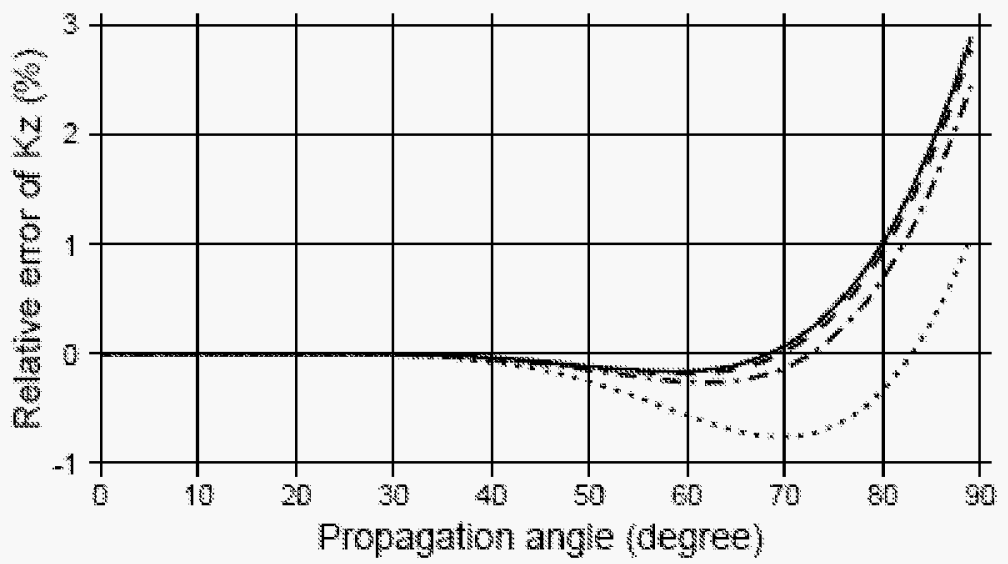

Figure 4. The relative errors of the approximate propagator with constant percentage perturbations of $100 \%$ (the dotted curve), $200 \%$ (the dot-dashed curve), $300 \%$ (the dashed curve), $400 \%$ (the solid curve).

\subsection{Example of Application to Imaging}

This propagator can be used for both seismic modeling and imaging. Here we test its imaging performance. The data set is for testing its ability of imaging steeply-inclined reflectors embedded in a VTI medium with strong perturbations. As shown in Figure 5, the model has reflectors with dip angles of $0^{\circ}, 15^{\circ}, 30^{\circ}, 45^{\circ}, 60^{\circ}, 75^{\circ}$ and $90^{\circ}$. The grid dimensions of the model are 100 gridlines in depth and 700 gridlines in the horizontal, with $\mathrm{dx}=\mathrm{dz}=12.19 \mathrm{~m}$. A similar model is used by Le Rousseau (1997). The anisotropy of the model is characterized by $\delta=0.3, \varepsilon=0.2$ The qP-wave velocity model is composed of the background velocity of $701 \mathrm{~m} / \mathrm{s}$ and vertical and lateral gradients of 0.6 $\mathrm{s}^{-1}$ and $1.0 \mathrm{~s}^{-1}$. The largest velocity perturbation at the model surface (the first thin-slab) is $730 \%$; the largest velocity perturbation at the model bottom (the last thin-slab) is $267 \%$. A Kirchhoff modeling method for a factorized TI medium (Alkhalifah, 1995) was used to generate a zero offset section with the central frequency of $8.5 \mathrm{~Hz}$. As shown in Figure 6, the synthetic section had 700 traces and 401 samples per trace. The sampling interval was $10 \mathrm{~ms}$. Imaging results are shown in Figure 7. Figure 7a shows that when the anisotropy of the model is ignored, the isotropic propagator gives a poor image. The steep reflectors are seriously under-migrated and the energy at the diffraction points of the structures is not focused. The reflectors are well imaged when the propagator takes the anisotropy into account (Figure 7b). This example demonstrates that the one-way propagator in VTI media has excellent performance for imaging steep reflectors in strongly heterogeneous VTI media. 


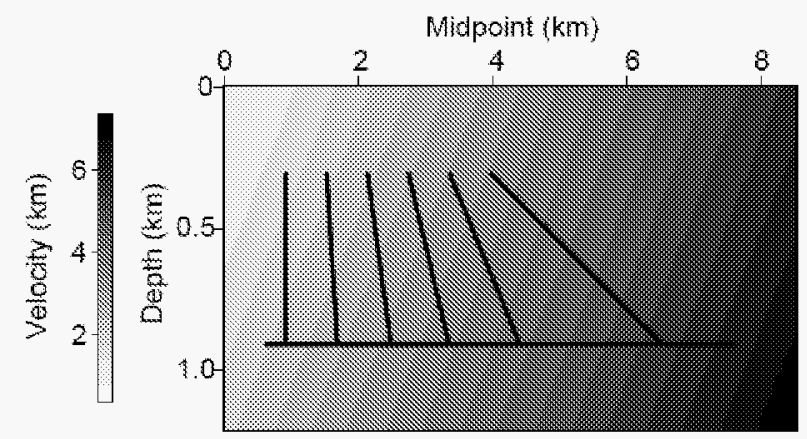

Figure 5. A VTI model with strong lateral velocity variations and steeply-inclined reflectors with dip angles of $0^{\circ}, 15^{\circ}, 30^{\circ}, 45^{\circ}, 60^{\circ}, 75^{\circ}$ and $90^{\circ}$ degrees.

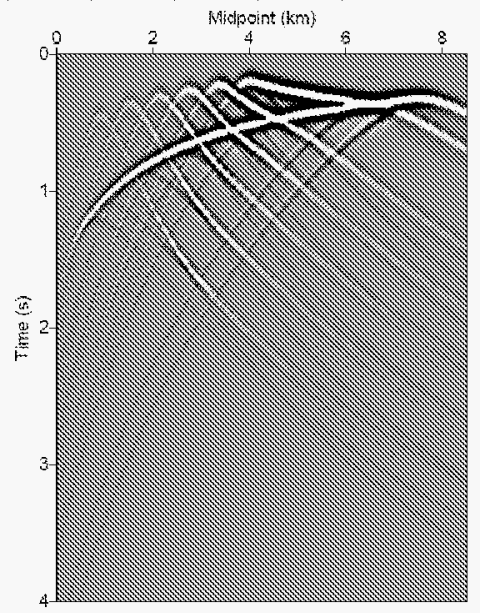

Figure 6. A zero offset section simulated with the model shown in Figure 5 using a Kirchhoff modeling method for a factorized TI medium. The section had 700 traces and 401 samples per trace. The sampling interval is $10 \mathrm{~ms}$.

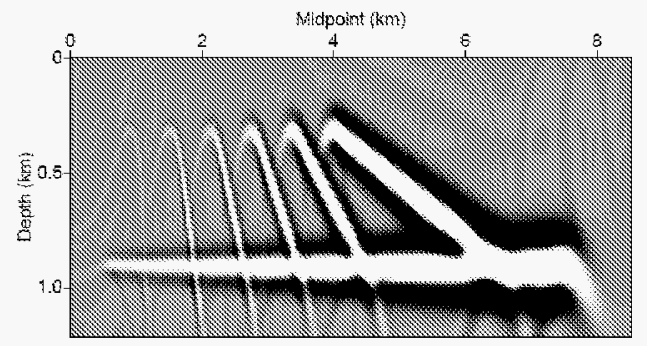

(a)

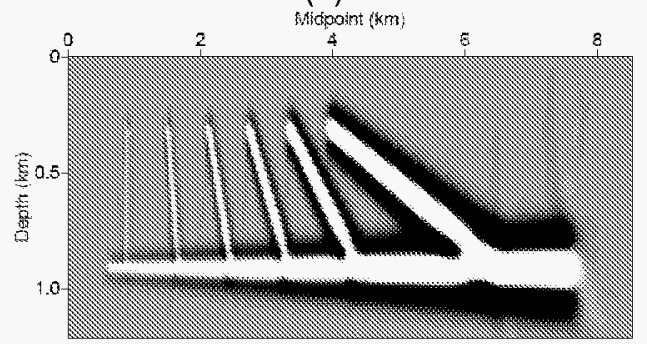

(b)

Figure 7. Depth images of the section shown in Figure 7 migrated with an isotropic propagator (a), and with the anisotropic propagator (b). 


\subsection{Some Discussions}

The scalar qP-wave propagator for a VTI medium was derived by introducing wave potentials into the full elastic wave equations and applying the generalized screen approximation. The propagation in the background medium is accomplished in the wavenumber domain, while the interaction with heterogeneities, including application of the phase-screen correction and the wide-angle correction, is implemented in the space domain. The propagator can be used for complex VTI media. Results show that the propagator is accurate for strong perturbations of velocity and anisotropy parameters even at large propagation angles. The propagator can be used for $\mathrm{qP}$-wave field simulation and imaging/migration. We only give the propagator for a scalar $\mathrm{qP}$-wave in 2-D VTI media, the propagator for 3-D media can be derived similar to that for the 2-D case. With successive application in several directions, the 2-D propagator may be used for a 3-D medium. 


\section{IMPROVEMENT TO ELASTIC GENERALIZED SCREEN PROPAGATOR}

\subsection{Dual-Domain Thin-Slab Formulation}

The dual-domain expressions for $\mathrm{P}$ to $\mathrm{P}$ scattered displacement fields in isotropic elastic media can be obtained as ( $\mathrm{Wu}, 1996, \mathrm{Wu}$ and $\mathrm{Wu}, 2003 \mathrm{a})$

$$
\begin{aligned}
\mathbf{U}^{P P}\left(K_{T}, z^{*}\right) & =\frac{i k_{\alpha}^{2}}{2 \gamma_{\alpha}} \int_{z^{\prime}}^{z_{1}} d z e^{i k_{z}^{\alpha}\left(z^{*}-z\right)} \times \\
& \left\{\hat{k}_{\alpha} \hat{k}_{\alpha} \cdot \iint d^{2} \mathbf{x}_{T} e^{-i \mathbf{K}_{T} \cdot \mathbf{x}_{T}} \frac{\delta \rho\left(\mathbf{x}_{T}, z\right)}{\rho} \mathbf{u}_{\alpha}^{f}\left(\mathbf{x}_{T}, z\right)\right. \\
& -\hat{k}_{\alpha} \iint d^{2} \mathbf{x}_{T} e^{-i \mathbf{K}_{T} \cdot \mathbf{x}_{T}} \frac{\delta \lambda\left(\mathbf{x}_{T}, z\right)}{\lambda+2 \mu} \frac{1}{i k_{\alpha}} \nabla \cdot \mathbf{u}_{\alpha}^{f}\left(\mathbf{x}_{T}, z\right) \\
& \left.-\hat{k}_{\alpha}\left(\hat{k}_{\alpha} \hat{k}_{\alpha}\right): \iint d^{2} \mathbf{x}_{T} e^{-i \mathbf{K}_{T} \cdot \mathbf{x}_{T}} \frac{\delta \mu\left(\mathbf{x}_{T}, z\right)}{\lambda+2 \mu} \frac{1}{i k_{\alpha}} \varepsilon_{\alpha}^{f}\left(\mathbf{x}_{T}, z\right)\right\}
\end{aligned}
$$

with $k_{z}^{\alpha}=+\gamma_{\alpha}$ for forescattering and $k_{z}^{\alpha}=-\gamma_{\alpha}$ for backscattering, and $\hat{k}_{\alpha}=\left(\mathbf{K}_{T}, k_{z}^{\alpha}\right) / k_{\alpha}$. Note that we replaced $\rho_{0}, \lambda_{0}, \mu_{0}$ in denominators by $\rho=\rho_{0}+\delta \rho, \lambda=\lambda_{0}+\delta \lambda$ and $\mu=\mu_{0}+\delta \mu$. This replacement is the result of asymptotic matching between the Born approximation for large-angle scattering and the $\mathrm{h}$-f asymptotic travel-time (phase) for forward propagation. It is proved ( $\mathrm{Wu}$ and $\mathrm{Wu}, 2003 \mathrm{a}$ ) that with this replacement (asymptotic matching), the phase-shift in the exact forward direction is accurate and the phase error for small angles is reduced compared with Born approximation. In the meanwhile, the phase error for large angle scattering is much smaller than that of the phase screen approximation.

In equation (1) $\mathbf{u}_{\alpha}^{f}\left(\mathbf{x}_{T}, z\right), \nabla \cdot \mathbf{u}_{\alpha}^{f}\left(\mathbf{x}_{T}, z\right)$ and $\varepsilon_{\alpha}^{f}\left(\mathbf{x}_{T}, z\right)$ can be calculated by:

$$
\begin{gathered}
\mathbf{u}_{\alpha}^{f}\left(\mathbf{x}_{T}, z\right)=\frac{1}{4 \pi} \iint d^{2} \mathbf{K}_{T}^{\prime} e^{i \mathbf{K}_{T}^{\prime} \cdot \mathbf{x}_{T}} u_{\alpha}^{0}\left(\mathbf{K}_{T}^{\prime}\right) e^{i \gamma_{\alpha}^{\prime}\left(z-z^{\prime}\right)} \\
\frac{1}{i k_{\alpha}} \nabla \cdot \mathbf{u}_{\alpha}^{f}\left(\mathbf{x}_{T}, z\right)=\frac{1}{4 \pi} \iint d^{2} \mathbf{K}_{T}^{\prime} e^{i \mathbf{K}_{T}^{\prime} \cdot \mathbf{x}_{T}} \hat{k}_{\alpha}^{\prime} \cdot \mathbf{u}_{\alpha}^{0}\left(\mathbf{K}_{T}^{\prime}\right) e^{i \gamma_{\alpha}^{\prime}\left(z^{-z^{\prime}}\right)} \\
\frac{1}{i k_{\alpha}} \varepsilon_{\alpha}^{f}\left(\mathbf{x}_{T}, z\right)=\frac{1}{4 \pi} \iint d^{2} \mathbf{K}_{T}^{\prime} e^{i \mathbf{K}_{T}^{\prime} \cdot \mathbf{x}_{T}} \frac{1}{2}\left[\hat{k}_{\alpha}^{\prime} \mathbf{u}_{\alpha}^{0}\left(\mathbf{K}_{T}^{\prime}\right)+\hat{k}_{\alpha}^{\prime} \mathbf{u}_{\alpha}^{0}\left(\mathbf{K}_{T}^{\prime}\right)\right] e^{i \gamma_{\alpha}^{\prime}\left(z-z^{\prime}\right)} \\
=\frac{1}{4 \pi} \iint d^{2} \mathbf{K}_{T}^{\prime} e^{i \mathbf{K}_{T}^{\prime} \cdot \mathbf{x}_{T}} \hat{k}_{\alpha}^{\prime} \hat{k}_{\alpha}^{\prime} u_{\alpha}^{0}\left(\mathbf{K}_{T}^{\prime}\right) e^{i \gamma_{\alpha}^{\prime}\left(z-z^{\prime}\right)}
\end{gathered}
$$

where $u_{\alpha}^{0}\left(\mathbf{K}_{T}^{\prime}\right)=\left|\mathbf{u}_{\alpha}^{0}\left(\mathbf{K}_{T}^{\prime}\right)\right|$ and $\hat{k}_{\alpha}^{\prime}=\left(\mathbf{K}_{T}^{\prime}, \gamma_{\alpha}^{\prime}\right) / k_{\alpha}$.

For $\mathrm{P}$ to $\mathrm{S}$ scattering: 


$$
\begin{aligned}
\mathbf{U}^{P S}\left(\mathbf{K}_{T}, z^{*}\right)= & \frac{i k_{\beta}^{2}}{2 \gamma_{\beta}} \int_{z^{\prime}}^{z_{1}} d z e^{i k_{z}^{\beta}\left(z^{*}-z\right)}\left\{\left(\mathbf{I}-\hat{k}_{\beta} \hat{k}_{\beta}\right) \cdot \iint d^{2} \mathbf{x}_{T} e^{-i \mathbf{K}_{T} \cdot \mathbf{x}_{T}} \frac{\delta \rho\left(\mathbf{x}_{T}, z\right)}{\rho} \mathbf{l}_{\alpha}^{f}\left(\mathbf{x}_{T}, z\right)\right. \\
& \left.-\left(\mathbf{I}-\hat{k}_{\beta} \hat{k}_{\beta}\right) \cdot\left[\hat{k}_{\beta} \cdot \iint d^{2} \mathbf{x}_{T} e^{-i \mathbf{K}_{T} \cdot \mathbf{x}_{T}} 2 \frac{\delta \mu\left(\mathbf{x}_{T}, z\right)}{\mu} \frac{1}{i k_{\beta}} \varepsilon_{\alpha}^{f}\left(\mathbf{x}_{T}, z\right)\right]\right\}
\end{aligned}
$$

where $\hat{k}_{\beta}=\left(\mathbf{K}_{T}, k_{z}^{\beta}\right) / k_{\beta}$.

For $\mathrm{S}$ to $\mathrm{P}$ scattering:

$$
\begin{aligned}
\mathbf{U}^{S P}\left(\mathbf{K}_{T}, z^{*}\right)= & \frac{i k_{\alpha}^{2}}{2 \gamma_{\alpha}} \int_{z}^{z_{1}} d z e^{i k_{z}^{\alpha}\left(z^{*}-z\right)}\left\{\hat{k}_{\alpha} \hat{k}_{\alpha} \cdot \iint d^{2} \mathbf{x}_{T} e^{-i \mathbf{K}_{T} \cdot \mathbf{x}_{T}} \frac{\delta \rho\left(\mathbf{x}_{T}, z\right)}{\rho} \mathbf{u}_{\beta}^{f}\left(\mathbf{x}_{T}, z\right)\right. \\
& \left.-\left(\frac{k_{\alpha}}{k_{\beta}}\right) \hat{k}_{\alpha}\left(\hat{k}_{\alpha} \hat{k}_{\alpha}\right): \iint d^{2} \mathbf{x}_{T} e^{-i \mathbf{K}_{T} \cdot \mathbf{x}_{T}} 2 \frac{\delta \mu\left(\mathbf{x}_{T}, z\right)}{\mu} \frac{1}{i k_{\beta}} \varepsilon_{\beta}^{f}\left(\mathbf{x}_{T}, z\right)\right\}
\end{aligned}
$$

For $\mathrm{S}$ to $\mathrm{S}$ scattering:

$$
\begin{aligned}
\mathbf{U}^{S S}\left(\mathbf{K}_{T}, z^{*}\right)= & \frac{i k_{\beta}^{2}}{2 \gamma_{\beta}} \int_{z}^{z_{1}} d z e^{i k_{z}^{\beta}\left(z^{*}-z\right)}\left\{\left(\mathbf{I}-\hat{k}_{\beta} \hat{k}_{\beta}\right) \cdot \iint d^{2} \mathbf{x}_{T} e^{-i \mathbf{K}_{T} \cdot \mathbf{x}_{T}} \frac{\delta \rho\left(\mathbf{x}_{T}, z\right)}{\rho} \mathbf{u}_{\beta}^{f}\left(\mathbf{x}_{T}, z\right)\right. \\
& \left.-\left(\mathbf{I}-\hat{k}_{\beta} \hat{k}_{\beta}\right) \cdot\left[\hat{k}_{\beta} \cdot \iint d^{2} \mathbf{x}_{T} e^{-i \mathbf{K}_{T} \cdot \mathbf{x}_{T}} 2 \frac{\delta \mu\left(\mathbf{x}_{T}, z\right)}{\mu} \frac{1}{i k_{\beta}} \varepsilon_{\beta}^{f}\left(\mathbf{x}_{T}, z\right)\right]\right\}
\end{aligned}
$$

In (4) and (5) $\mathbf{u}_{\beta}^{f}\left(\mathbf{x}_{T}, z\right)$ and $\varepsilon_{\beta}^{f}\left(\mathbf{x}_{T}, z\right)$ can be calculated by

$$
\begin{gathered}
\mathbf{u}_{\beta}^{f}\left(\mathbf{x}_{T}, z\right)=\frac{1}{4 \pi^{2}} \iint d^{2} \mathbf{K}_{T}^{\prime} e^{i \mathbf{K}_{T}^{\prime} \cdot \mathbf{x}_{T}} \mathbf{u}_{\beta}^{0}\left(\mathbf{K}_{T}^{\prime}\right) e^{i \gamma_{\beta}^{\prime}\left(z-z^{\prime}\right)} \\
\frac{1}{i k_{\beta}} \varepsilon_{\beta}^{f}\left(\mathbf{x}_{T}, z\right)=\frac{1}{4 \pi^{2}} \iint d^{2} \mathbf{K}_{T}^{\prime} e^{i \mathbf{K}_{T}^{\prime} \cdot \mathbf{x}_{T}} \frac{1}{2}\left[\hat{k}_{\beta}^{\prime} \mathbf{u}_{\beta}^{0}\left(\mathbf{K}_{T}^{\prime}\right)+\mathbf{u}_{\beta}^{0}\left(\mathbf{K}_{T}^{\prime}\right) \hat{k}_{\beta}^{\prime}\right] e^{i \gamma_{\beta}^{\prime}\left(z-z^{\prime}\right)}
\end{gathered}
$$

where $\hat{k}_{\beta}^{\prime}=\left(\mathbf{K}_{T}^{\prime}, \hat{\gamma}_{\beta}^{\prime}\right) / k_{\beta}$.

From equations (1) to (6), we see that the leading-order interactions between incident fields and heterogeneities are expressed in three-dimensional volume integrals. Also the scattered and incident wavenumbers are coupled with each other. So the computation of these equations are still intensive. In this section the parts of the integration over $z$ in the equations are analytically estimated. Assume that the slab for each marching step is thin enough that the parameters (velocity and density) can be approximately taken as invariant along $z$, the integration with respect to $z$ in equation (1) can be calculated as

$$
\int_{z^{\prime}}^{z_{1}} d z e^{i k_{z}^{\alpha}\left(z^{*}-z\right)+i \gamma_{\alpha}^{\prime}\left(z-z^{\prime}\right)} \begin{cases}\Delta z e^{i\left(\gamma_{\alpha}+\gamma_{\alpha}^{\prime}\right) \Delta z / 2} \operatorname{sinc}\left[\frac{\gamma_{\alpha}-\gamma_{\alpha}^{\prime}}{2} \Delta z\right] & \text { for forescattering }\left(z^{*}=z_{1}\right) \\ \Delta z e^{i\left(\gamma_{\alpha}+\gamma_{\alpha}^{\prime}\right) \Delta z / 2} \operatorname{sinc}\left[\frac{\gamma_{\alpha}+\gamma_{\alpha}^{\prime}}{2} \Delta z\right] & \text { for backscattering }\left(z^{*}=z^{\prime}\right)\end{cases}
$$

We see that the integration over $z$ has been done analytically; however, $\gamma_{\alpha}$ and $\gamma_{\alpha}^{\prime}$ are still coupled, which prevents the thin-slab method from fast computation. To decouple $\gamma_{\alpha}$ and $\gamma_{\alpha}^{\prime}$, we neglect the angular variation of amplitude factors but keep the phase 
information untouched by taking the approximation $\gamma_{\alpha}=\gamma_{\alpha}^{\prime}=k_{\alpha}$ for the amplitude factors in equation (7). This assumption is valid for the case where the small-angle scattering is dominant, and therefore the direction of the scattered waves are not far from the incident direction. Under this approximation, equation (7) becomes

$$
\int_{z^{\prime}}^{z_{1}} d z e^{i k_{z}^{\alpha}\left(z^{*}-z\right)+i \gamma_{\alpha}^{\prime}\left(z-z^{\prime}\right)} \approx\left\{\begin{array}{cc}
\Delta z e^{i\left(\gamma_{\alpha}+\gamma_{\alpha}^{\prime}\right) \Delta z / 2} & \text { for forescattering }\left(z^{*}=z_{1}\right) \\
\Delta z e^{i\left(\gamma_{\alpha}+\gamma_{\alpha}^{\prime}\right) \Delta z / 2} \operatorname{sinc}\left(k_{\alpha} \Delta z\right) & \text { for backscattering }\left(z^{*}=z^{\prime}\right)
\end{array}\right. \text {. }
$$

For the scattered fields P-S, S-P and S-S, similar approximations can be obtained as follows. For P-S scattering,

$$
\int_{z^{\prime}}^{z_{1}} d z e^{i k_{z}^{\beta}\left(z^{*}-z\right)+i \gamma_{\alpha}^{\prime}\left(z-z^{\prime}\right)} \approx\left\{\begin{array}{ll}
\Delta z e^{i\left(\gamma_{\alpha}^{\prime}+\gamma_{\beta}\right) \Delta z / 2} \operatorname{sinc}\left[\left(k_{\alpha}-k_{\beta}\right) \Delta z / 2\right] & \text { for forescattering }\left(z^{*}=z_{1}\right) \\
\Delta z e^{i\left(\gamma_{\alpha}^{\prime}+\gamma_{\beta}\right) \Delta z / 2} \operatorname{sinc}\left[\left(k_{\alpha}+k_{\beta}\right) \Delta z / 2\right] & \text { for backscattering }\left(z^{*}=z^{\prime}\right)
\end{array} .\right.
$$

For S-S scattering,

$$
\int_{z^{\prime}}^{z_{1}} d z e^{i k_{z}^{\beta}\left(z^{*}-z\right)+i \gamma_{\beta}^{\prime}\left(z-z^{\prime}\right)} \approx\left\{\begin{array}{cc}
\Delta z e^{i\left(\gamma_{\beta}+\gamma_{\beta}^{\prime}\right) \Delta z / 2} & \text { for forescattering }\left(z^{*}=z_{1}\right) \\
\Delta z e^{i\left(\gamma_{\beta}+\gamma_{\beta}^{\prime}\right) \Delta z / 2} \operatorname{sinc}\left(k_{\beta} \Delta z\right) & \text { for backscattering }\left(z^{*}=z^{\prime}\right)
\end{array} .\right.
$$

The corresponding factor for S-P scattering is the same as for the P-S scattering. After the integration over $\mathrm{z}$, the integration over transverse plane $\mathbf{x}_{T}$ in (1) - (6) can now be carried out by the FFT. In order to further expedite the computation, we group the scattered fields (1) to (6) into $\mathbf{U}^{P}\left(\mathbf{K}_{T}, z^{*}\right)=\mathbf{U}^{P P}\left(\mathbf{K}_{T}, z^{*}\right)+\mathbf{U}^{S P}\left(\mathbf{K}_{T}, z^{*}\right)$, and $\mathbf{U}^{S}\left(\mathbf{K}_{T}, z^{*}\right)=\mathbf{U}^{P S}\left(\mathbf{K}_{T}, z^{*}\right)+\mathbf{U}^{S S}\left(\mathbf{K}_{T}, z^{*}\right)$, i.e.

$$
\begin{aligned}
& \mathbf{U}^{P}\left(\mathbf{K}_{T}, z^{\star}\right)= \frac{i k_{\alpha}^{2}}{2 \gamma_{\alpha}} e^{i \gamma_{\alpha} \Lambda z / 2} \Delta z \hat{k}_{\alpha}\left\{\hat{k}_{\alpha} \cdot \iint d^{2} \mathbf{x}_{T} e^{-i \mathbf{K}_{T} \mathbf{x}_{T}} \frac{\delta \rho\left(\mathbf{x}_{T}\right)}{\rho}\left[\eta^{P P} \mathbf{u}_{\alpha}^{f}\left(\mathbf{x}_{T}\right)+\eta^{S P} \mathbf{u}_{\beta}^{f}\left(\mathbf{x}_{T}\right)\right]\right. \\
& \quad-\iint d^{2} \mathbf{x}_{T} e^{-i \mathbf{K}_{T} \cdot \mathbf{x}_{T}} \frac{\delta \lambda\left(\mathbf{x}_{T}\right)}{\lambda+2 \mu} \frac{1}{i k_{\alpha}} \nabla \cdot\left[\eta^{P P} \mathbf{u}_{\alpha}^{f}\left(\mathbf{x}_{T}\right)\right] \\
&\left.-\left(\hat{k}_{\alpha} \hat{k}_{\alpha}\right): \iint d^{2} \mathbf{x}_{T} e^{-i \mathbf{K}_{T} \cdot \mathbf{x}_{T}} \frac{2 \delta \mu\left(\mathbf{x}_{T}\right)}{\lambda+2 \mu} \frac{1}{i k_{\alpha}}\left[\eta^{P P} \varepsilon_{\alpha}^{f}\left(\mathbf{x}_{T}\right)+\eta^{S P} \varepsilon_{\beta}^{f}\left(\mathbf{x}_{T}\right)\right]\right\}, \\
& \mathbf{U}^{S}\left(\mathbf{K}_{T}, z^{*}\right)= \frac{i k_{\beta}^{2}}{2 \gamma_{\beta}} e^{i \gamma_{\beta} \Delta z / 2} \Delta z\left(\mathrm{I}-\hat{k}_{\beta} \hat{k}_{\beta}\right) \cdot\left\{\iint d^{2} \mathbf{x}_{T} e^{-i \mathbf{K}_{T} \cdot \mathbf{x}_{T}} \frac{\delta \rho\left(\mathbf{x}_{T}\right)}{\rho}\left[\eta^{P S} \mathbf{u}_{\alpha}^{f}\left(\mathbf{x}_{T}\right)+\eta^{S S} \mathbf{u}_{\beta}^{f}\left(\mathbf{x}_{T}\right)\right]\right. \\
&\left.-\hat{k}_{\beta} \cdot \iint d^{2} \mathbf{x}_{T} e^{-i \mathbf{K}_{T} \mathbf{x}_{T}} \frac{2 \delta \mu\left(\mathbf{x}_{T}\right)}{\mu} \frac{1}{i k_{\beta}}\left[\eta^{P S} \varepsilon_{\alpha}^{f}\left(\mathbf{x}_{T}\right)+\eta^{S S} \varepsilon_{\beta}^{f}\left(\mathbf{x}_{T}\right)\right]\right\} .
\end{aligned}
$$

where $z^{*}\left(z^{*}=z^{\prime}\right.$ or $\left.z^{*}=z_{1}\right)$ indicates the position of the receiver plane. The modulation factors $\eta^{P P}, \eta^{S P}=\eta^{P S}$ and $\eta^{S S}$ are 


$$
\begin{aligned}
& \begin{aligned}
\eta^{P P} & =\left\{\begin{array}{cc}
1 & \text { for forescattering } \\
\operatorname{sinc}\left(k_{\alpha} \Delta z\right) & \text { for backscattering }
\end{array}\right. \\
\eta^{P S} & =\left\{\begin{array}{ll}
\operatorname{sinc}\left[\left(k_{\alpha}-k_{\beta}\right) \Delta z / 2\right] & \text { for forescattering } \\
\operatorname{sinc}\left[\left(k_{\alpha}+k_{\beta}\right) \Delta z / 2\right] & \text { for backscattering }
\end{array},\right.
\end{aligned} \\
& \eta^{S S}=\left\{\begin{array}{cc}
1 & \text { for forescattering } \\
\operatorname{sinc}\left(k_{\beta} \Delta z\right) & \text { for backscattering }
\end{array} .\right.
\end{aligned}
$$

Note that the factors $e^{i \gamma_{\alpha}\left(z-z^{\prime}\right)}$ and $e^{i \gamma_{\beta}\left(z-z^{\prime}\right)}$ have been replaced by $e^{i \gamma_{\alpha} \Lambda z / 2}$ and $e^{i \gamma_{\beta} \Lambda z / 2}$ for calculating the background fields. The phase matching (asymptotic matching) has been applied in equations (11) and (12).

Under the above approximations, the thin-slab formulation may be implemented by the procedures as used in the complex screen method (Wu, 1994; Xie and Wu, 2001). First, the whole medium is sliced into appropriate thin-slabs along the overall propagation direction. Weak scattering conditions hold for each thin-slab and the parameters can be considered invariant within each thin-slab in the preferred propagation direction. Suppose that all incident fields at the entrance of each thin-slab are given in the wavenumber domain. The implementation procedures may be summarized as follows: 1. Freely propagate in the wavenumber domain and calculate the primary fields, the divergence of incident $P$ wave, and strains (equations 2 and 6).

2. Inverse-FFT the primary fields, the divergence and strains into the space domain, and then calculate the distorted fields (the space domain functions before the FT in equations 11-12).

3. Calculate the forescattered fields at the thin-slab exit, and add the forescattered fields to the primary fields to form the total fields as the incident fields for the next thin-slab. In the same time backscattered fields at the thin-slab entrance are also calculated.

4. Continue the procedures 1 to 3 iteratively until the last thin-slab, ant the total transmitted fields are obtained at the exit of the last thin-slab.

5. Propagate back the backscattered fields from the last to the first thin-slabs using a similar iterative procedure as step 1 to 3 , and sum up all backscattered fields generated by each thin-slab to get the total reflected fields at the surface.

Let us estimate the computation speed. Most calculations involve only fast Fourier transforms. The total computation time can be estimated from the time used in calling FFTs. Taking the complex screen method as a reference, for a 2-D case, the thin-slab method needs 11 inverse FFTs and 11 forward FFTs, while the complex screen method needs 5 inverse FFTs and 7 forward FFTs; For a 3-D case, the thin-slab method needs 19 inverse FFTs and 19 forward FFTs, while the complex screen method needs 7 inverse FFTs and 10 forward FFTs. The thin-slab method takes about twice time as much as the complex screen method does, but is still much faster than the full wave methods. We will see a comparison of computation time between the thin-slab method and finite difference method in the next section. 


\subsection{Numerical Tests for the Elastic Thin-Slab Method}

\subsubsection{Reflection Coefficient Calculations.}

The following examples show the angle dependence of amplitudes (reflection coefficients) calculated by the thin-slab method. The model used is defined on a $2048 \times 200$ rectangular grid. The grid spacing in the horizontal direction is $16 \mathrm{~m}$ and in the vertical direction is $4 \mathrm{~m}$. A horizontal interface is introduced in the middle of the model. The upper layer has $\alpha=3.6 \mathrm{~km} / \mathrm{s}, \beta=2.08 \mathrm{~km} / \mathrm{s}$, and $\rho=2 \mathrm{~g} / \mathrm{cm}^{3}$, which is taken as background medium. The lower layer has different $\mathrm{P}$ and $\mathrm{S}$ wave velocities relative to the upper layer. A $15 \mathrm{~Hz}$ plane $\mathrm{P}$ wave (or $\mathrm{S}$ wave) is incident on the interface at different angles. To enhance the stability in the calculation of reflection coefficients, we chose 500 samples (displacement amplitudes in the space domain) at the center of the model for both the incident and reflected fields and calculate their means respectively. Reflection/conversion coefficients are defined by the ratio of the reflected amplitudes to the incident amplitudes at the same receiver plane. Figure 1 shows the reflection/conversion coefficients versus angle of incidence with a perturbation of $10 \%$ for both $\mathrm{P}$ and $\mathrm{S}$ wave velocities. The theoretical reflection/conversion coefficients (dashed lines) are also given as references. The upper panel corresponds to $\mathrm{P}$ wave incidence and the lower panel, $S$ wave incidence. The angles of incidence of $S$ wave are limited to the critical angle of S-P converted wave. Both results agree well with the theory for a wide range of incident angles $\left(55^{\circ}\right.$ for $\mathrm{P}$ wave incidence, and near critical angle $-32^{\circ}$ for $\mathrm{S}$ wave incidence).

Figures 2 to 4 show similar results as shown in Figure 1 but with different perturbations of $-10 \%, 20 \%$ and $-20 \%$, respectively. Figure 2 corresponds to a negative velocity perturbation. For $\mathrm{P}$ wave incidence, no critical angle exists. However, errors occur for large angles of incidence ( $>65^{\circ}$ for $\mathrm{P}$ wave incidence). This is limited by the ability of one-way propagator to handle extra wide-angle waves. For $\mathrm{S}$ wave incidence, both results are in good agreement up to the critical angle. Figure 3 corresponds to a perturbation of $20 \%$ for both $\mathrm{P}$ and $\mathrm{S}$ wave velocities. For a small angle of incidence $\left(40^{\circ}\right.$ for $\mathrm{P}$ wave incidence and $20^{\circ}$ for $\mathrm{S}$ wave incidence), the thin-slab results match the theoretical values. Comparing Figures 3 and 1, we see that the wide-angle capacity of the thin-slab method decreases as perturbations increase. Figure 4 corresponds to a perturbation of $-20 \%$ for both $\mathrm{P}$ and $\mathrm{S}$ wave velocities. 

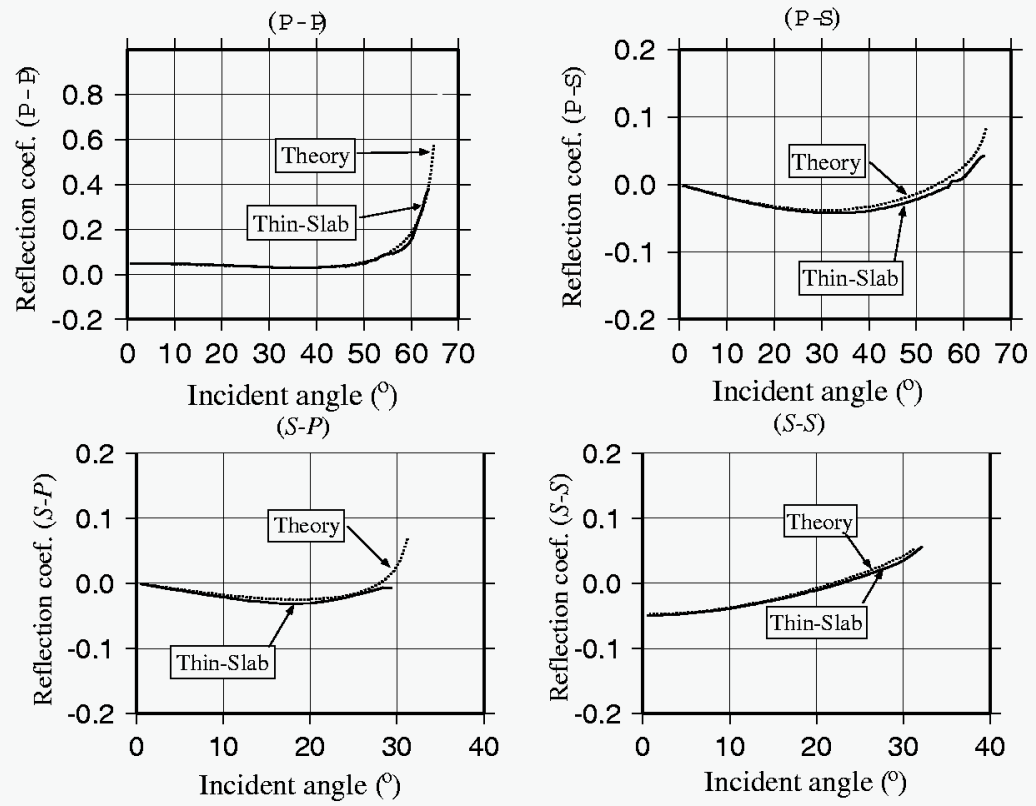

Figure 1. Comparisons of reflection coefficients calculated by the thin-slab method and the exact solutions. The upper panel corresponds to $\mathrm{P}$ wave and the lower panel to $\mathrm{S}$ wave incidences respectively. The bottom layer of the model has a $10 \%$ velocity perturbations for both $\mathrm{P}$ and $\mathrm{S}$ waves compared to the top layer.
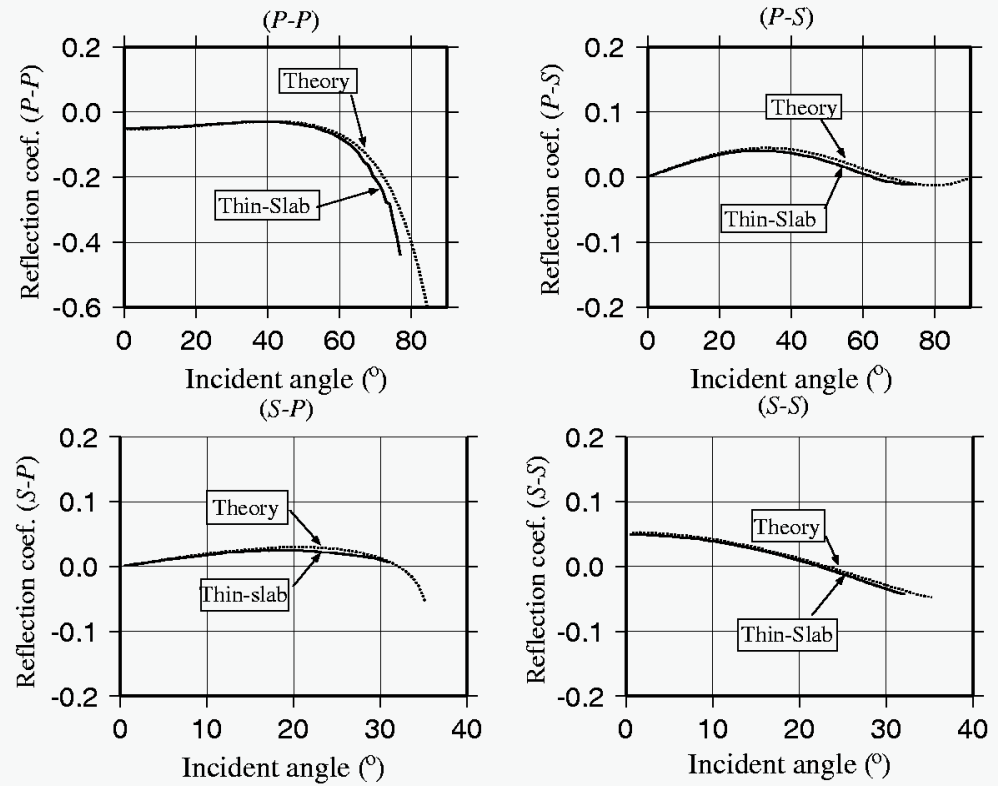

Figure 2: Comparisons of reflection coefficients calculated by the thin-slab method and the exact solutions. The perturbation of the bottom layer is $-10 \%$ with respect to the top layer for both $\mathrm{P}$ and $\mathrm{S}$ wave velocities. 

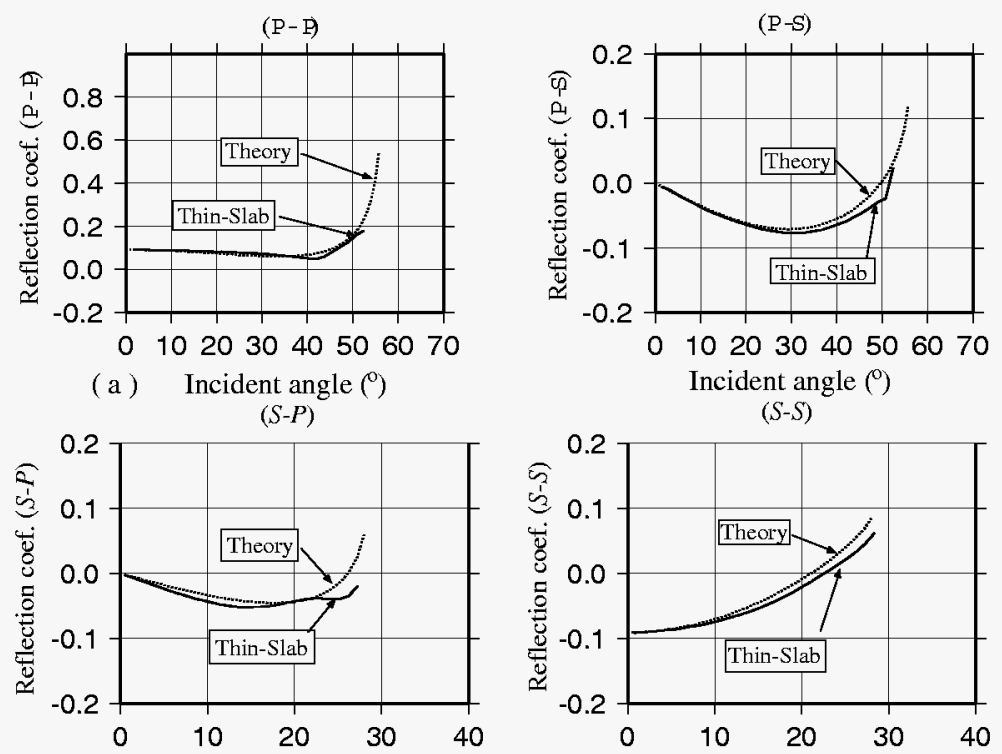

(b) Incident angle $($ )

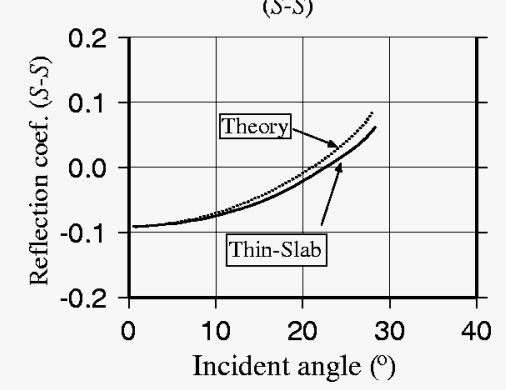

Figure 3. Comparisons of reflection coefficients calculated by the thin-slab method and the exact solutions. The perturbation of the bottom layer is $20 \%$ with respect to the top layer for both $\mathrm{P}$ and $\mathrm{S}$ wave velocities.
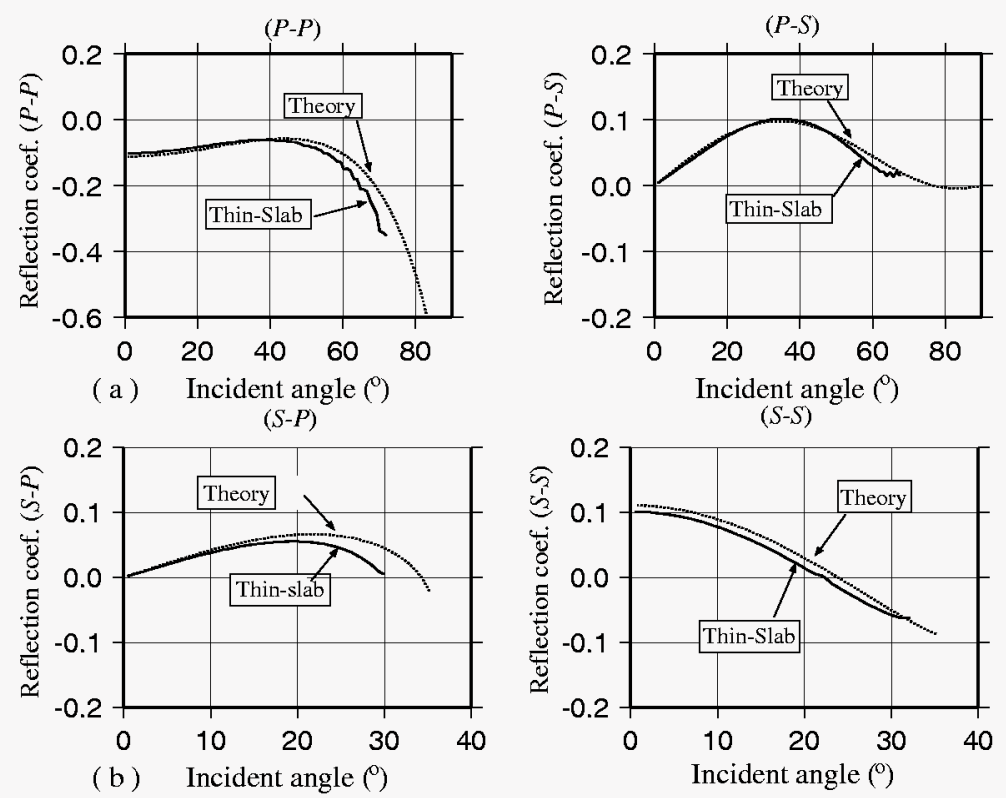

Figure 4. Comparisons of reflection coefficients calculated by the thin-slab method and the exact solutions. The perturbation of the bottom layer is $-20 \%$ with respect to the top layer for both $\mathrm{P}$ and $\mathrm{S}$ wave velocities.

\subsubsection{D Synthetic Seismograms.}

Synthetic seismograms are also generated to further demonstrate the capability of the thin-slab method. Figure 5 shows a 2-D model that is a vertical slice cut from the elastic 
French model (French, 1974). The model has a strong irregular interface that will generate large-angle reflections and scattering. The parameters of the background medium are taken as $\alpha_{0}=3.6 \mathrm{~km} / \mathrm{s}, \beta_{0}=2.08 \mathrm{~km} / \mathrm{s}$ and $\rho_{0}=2.2 \mathrm{~g} / \mathrm{cm}^{3}$. The layer in black color has a perturbation of $-20 \%$ for both $\mathrm{P}$ and $\mathrm{S}$ wave velocities. Source and receiver geometry are also shown in the figure. A Ricker wavelet with a dominant frequency of $20 \mathrm{~Hz}$ is used. For the thin-slab method, the spacing interval is $8 \mathrm{~m}$ in both horizontal and vertical directions. For the finite difference method, the spacing interval is $4 \mathrm{~m}$ and time interval is $0.5 \mathrm{~ms}$. The direct arrivals have been properly removed from the finite difference results. Figure 6 shows a comparison of the synthetic seismograms between the thin-slab method and finite difference method. Both amplitudes and arrival times are in good agreement up to large offsets $(\sim 1.4 \mathrm{~km})$ compared to the depth $(\sim 1 \mathrm{~km})$. For this example, the thin-slab method is about 57 times faster than the finite difference method. The factor will increase as the size of model increases, especially for 3D cases.

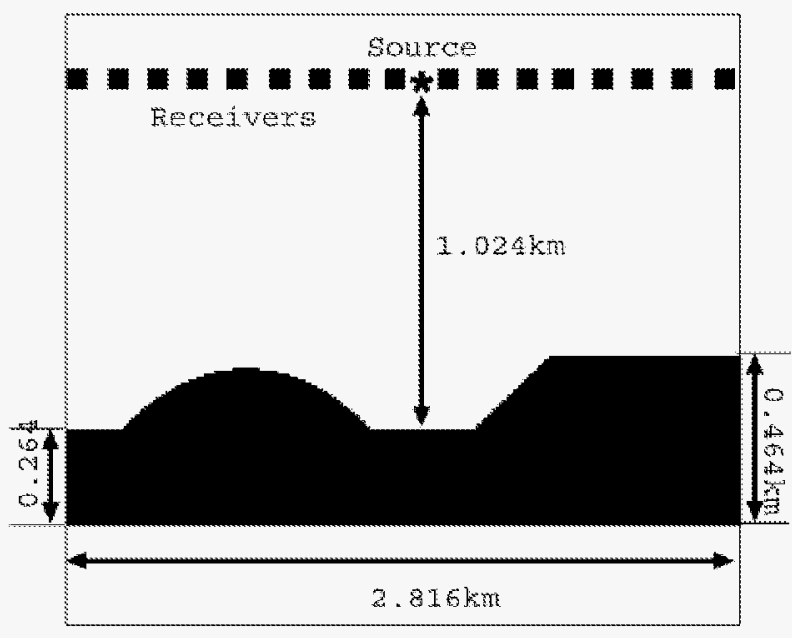

Figure 5. A 2-D heterogeneous model (French, 1974) with irregular interface used to test the validity and accuracy of the thin-slab method. Background medium has $\alpha_{0}=3.6 \mathrm{~km} / \mathrm{s}, \beta_{0}=2.08 \mathrm{~km} / \mathrm{s}$ and $\rho_{0}=2.2 \mathrm{~g} / \mathrm{cm}^{3}$. The layer in black color has a perturbation of $-20 \%$ for both $\mathrm{P}$ and $\mathrm{S}$ wave velocities.

\subsection{Generalized Screen Propagator with Intrinsic Attenuation}

The thin-slab propagator is a one-way elastic wave dual-domain propagator which can be used to calculate reflections based on the De Wolf approximation (multiple-

forescattering-singlebackscattering approximation). The quality factors Qp and Qs have been incorporated into the thin-slab propagator by introducing complex velocities $(\mathrm{Wu}$ and $\mathrm{Wu}, 1999,2003 \mathrm{~b}$ ). The resulting viscoelastic thin-slab propagator can be used to handle elastic wave propagation in models with intrinsic attenuation. Figure 7 shows the results of a validation of the modeling approach for the case of acoustic propagation. The model used for validation consists of two homogeneous half-spaces that are in contact. Each half space can be assigned a value of Q. Plane waves are incident from one half space onto the boundary with various angles of incidence. Waveforms received in the second half space are used to estimate the apparent $Q$ using the spectral ratio method. In 
the figure, Qo is Page 15 of 67 quality factor in the medium with the incident plane waves and $Q$ is that for the lower half space. The velocity perturbations $\delta \mathrm{v} / \mathrm{v}$ are labeled for each curve. From Figure $7 \mathrm{a}$ we see that for $\delta \mathrm{v} / \mathrm{v}=0$ the intrinsic attenuation of the medium is exactly matched for all incidence angles. For models with perturbations in either velocity or Q, quality factors are accurately estimated for small to moderate incident angles.
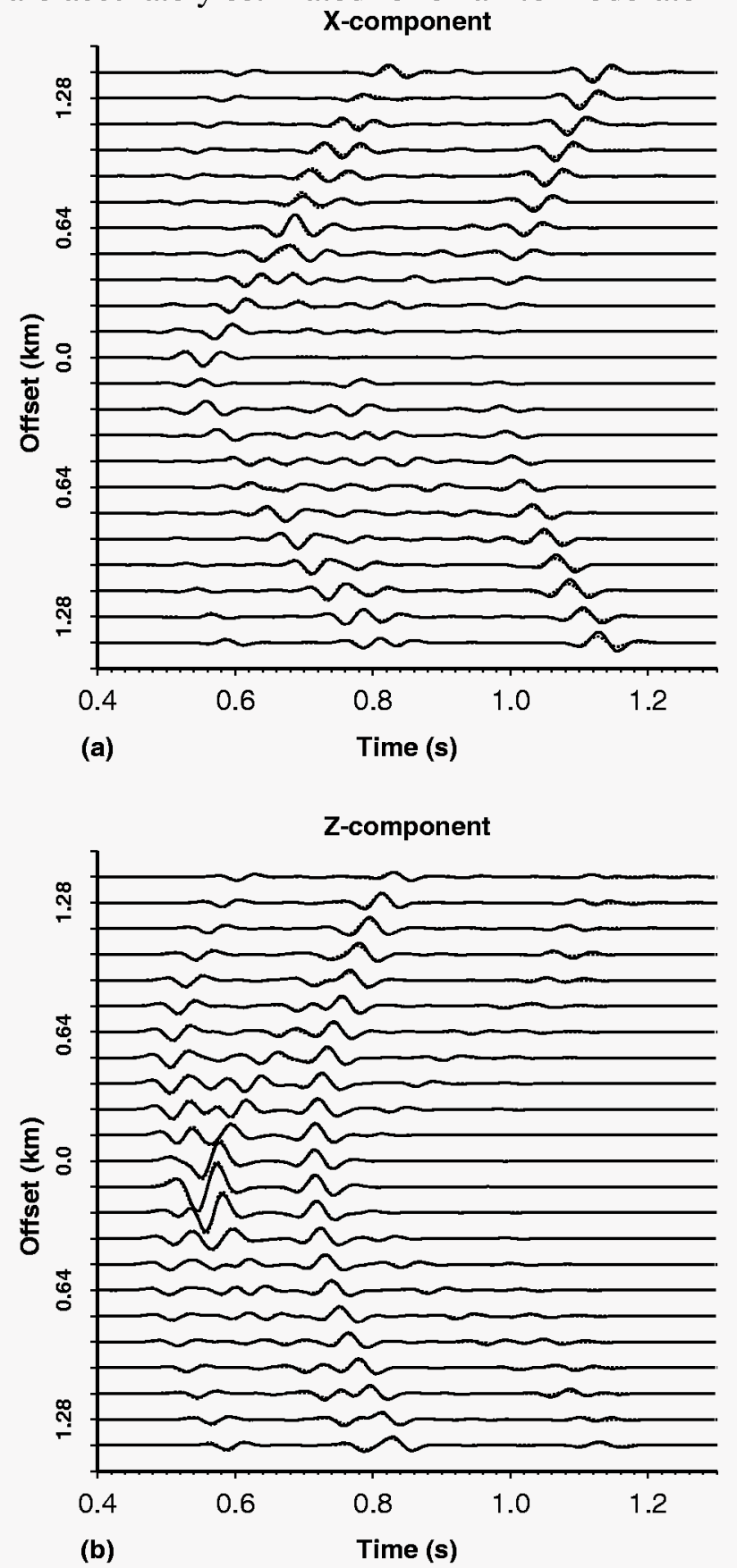

Figure 6. Synthetic seismograms calculated with finite difference (solid) and the thin-slab methods (dashed). Curves in (a) and (b) are horizontal and vertical components of displacement, respectively. 

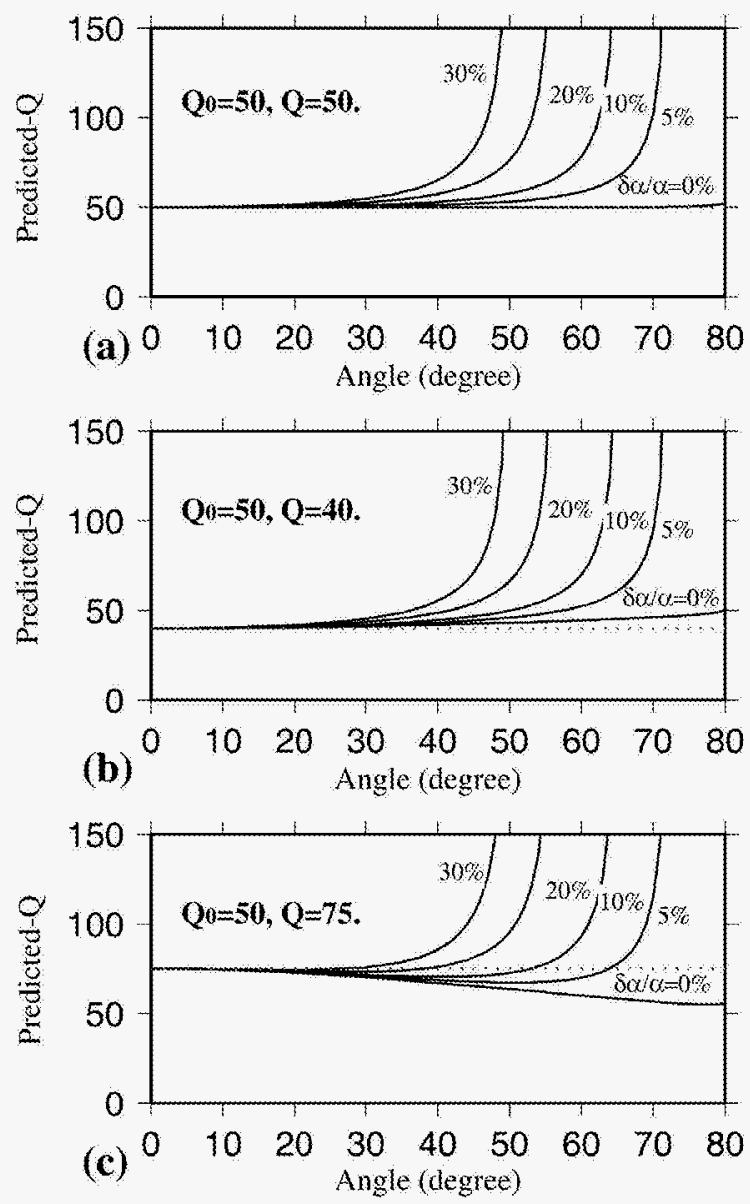

Figure 7. The predicted $Q$ values for models calculated using the viscoelastic thin-slab propagator. For models (a), (b) and (c), the Q values in the medium with the receivers are 50,40 and 75 respectively. The quality factor in the medium with the incident plane waves is $\mathbf{5 0}$ for all three models. The velocity perturbation $\mathrm{dv} / \mathrm{v}$ is labeled on each curve.

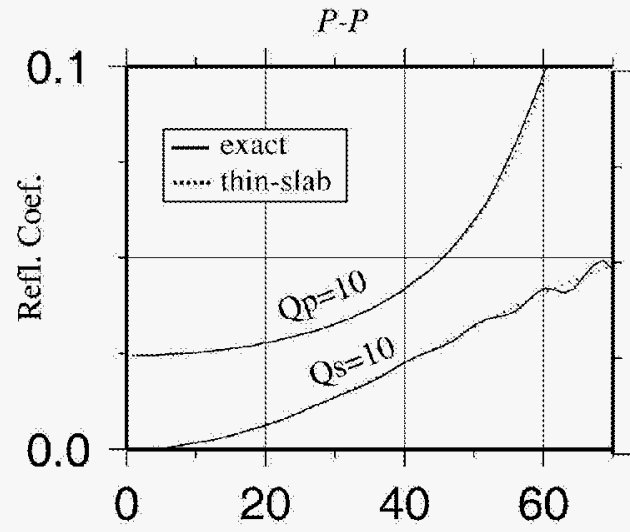

(a) Incident angle (degree)

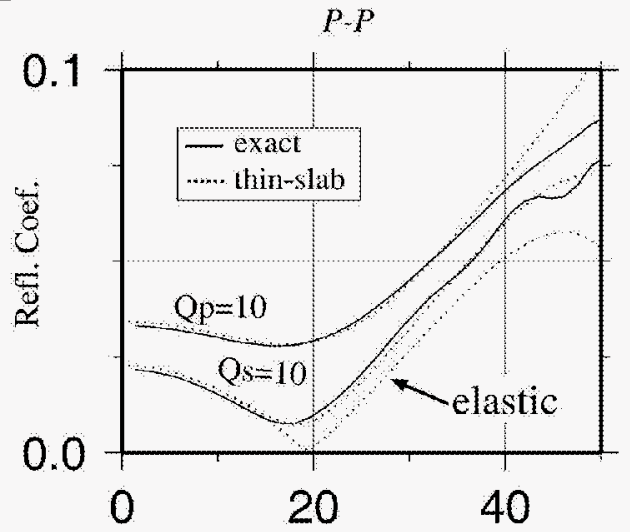

(b) Incident angle (degree)

Figure 8 . The P-P reflection coefficients at interfaces between an elastic half space and a viscoelastic half space. The solid lines are from exact solutions and the doted lines are from viscoelastic thin-slab solutions. (a) Interface between an elastic shale and a viscoelastic shale. The lower half space has either $\mathrm{Qp}=10$ or $\mathrm{Qs}=10$. (b) Similar to (a) except the lower half space is a viscoelastic oil sand. 
Figure 8 shows the P-P reflection coefficients for interfaces between an elastic half space and a viscoelastic half space. The values calculated by modeling using the elastic thinslab propagator agree well with those from an exact solution (e.g., Aki and Richards, 1980). 


\section{APPLICATIONS OF FAST ELASTIC-WAVE MODELING}

\subsection{AVO Modeling/Analysis}

Real geological media contain heterogeneities in P- and S- wave velocities, density, and spatial variations in attenuation. Compared with a homogeneous elastic model, these heterogeneities may have different effects on AVO response. The viscoelastic thin-slab method has demonstrated great potential for modeling these effects ( $\mathrm{Wu}$ and $\mathrm{Wu}, 2003 \mathrm{~b}$ ). Figure 1 shows the effects of heterogeneous Q and elastic parameters on AVO responses. Shown in the top panel of Figure 1 is the velocity model which includes random spatial variations in both elastic and anelastic parameters (from $\mathrm{Wu}$ and $\mathrm{Wu}, 2003 \mathrm{~b}$ ). The rest panels in Figure 1 show the AVO responses for shale and gasbearing sand contact (middle panel) and shale and oil-bearing sand contact (bottom panel). The AVO response is shown as curves for various cases for each type of material in the lower layer. Different cases are shown for various values of Qp and Qs in the upper layer, labeled as Qpo and Qso, and in the lower layer, which are labeled as Qp and Qs. The correlation lengths of the heterogeneities for both elastic and anelastic parameters are same. The RMS perturbations are $4 \%$ for elastic parameters and $25 \%$ for $\mathrm{Q}$. Source and receiver arrays are located at the top of the shale. The smooth lines represent the AVO responses for homogeneous attenuating media and the rough curves are those for the heterogeneous media. Comparing AVO responses from different models, the effects from different elastic and anelastic parameters are significant.

\subsection{Random Media Characterization and AVO Analysis}

Imaging provides the ability to determine the geometrical structure of the Earth. However, structure on some scales cannot be reliably determined from conventional seismic imaging so other approaches must be taken for characterization. Characterization of some portions of the structure can best be done using a random media approach. Such approaches provide valuable tools for understanding scattering and attenuation in the Earth (see e.g. Sato, Fehler, and Wu, 2002; Pride et al., 2003). We have used numerical modeling of wave propagation through random media along with various analytical approaches to better understand what characteristics of the medium can be inferred from analysis of seismic traces (Fehler, Sato, and Huang, 2000; Saito et al. 2003; Sato et al., 2003). We have recently been investigating an approach for using AVO analysis to characterize the spatial heterogeneity in a layer. Figure 2 shows an example of the application of the method. We consider random media that are similar to those shown in the top panel of Figure 1. We compare the spectrum of the spatial fluctuation of medium velocity, shown in red, with the spectrum of the spatial fluctuation in integrated trace amplitude measured along a receiver array. The figure shows that the two spatial spectra are coincident over the spatial wavenumber range from $.01 \mathrm{~km}-1$ to $1 \mathrm{~km}-1$. The departure of the two lines for spatial wavenumbers greater than $1 \mathrm{~km}-1$ is due to the band-limited source that was used for the numerical modeling (source was a $2 \mathrm{~Hz}$ Ricker wavelet for this example). 


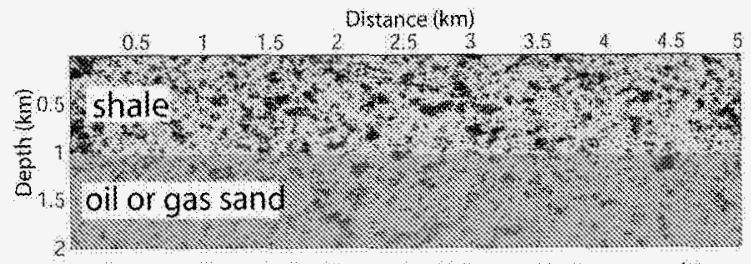

Reservor model with anelasticity and heterogeneities
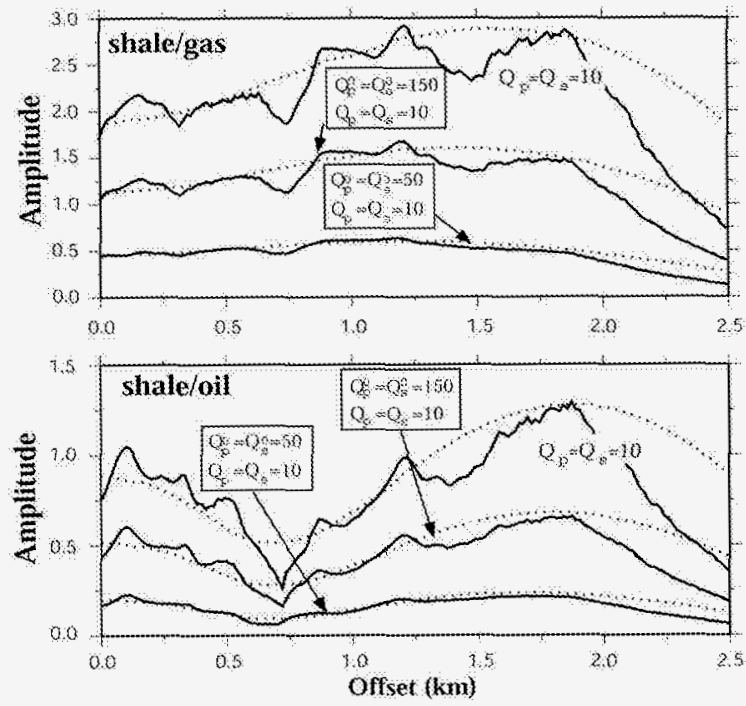

Figure 1. AVO modeling for viscoelastic heterogeneous reservoirs (oil or gas sand model). (Top) velocity model, (Middle) AVO response for the shale/gas interface, and (Bottom) AVO response for the shale/oil interface. $Q_{P}^{0}$ and $Q_{S}^{0}$ are the quality factors of $\mathrm{P}$ - and S-waves for the shale layer, and $Q_{P}$ and $Q_{S}$ are the quality factors of P- and Swaves for oil or gas sand layer. The smooth lines represent the AVO responses for homogeneous attenuating media and the rough curves are those for the heterogeneous media.

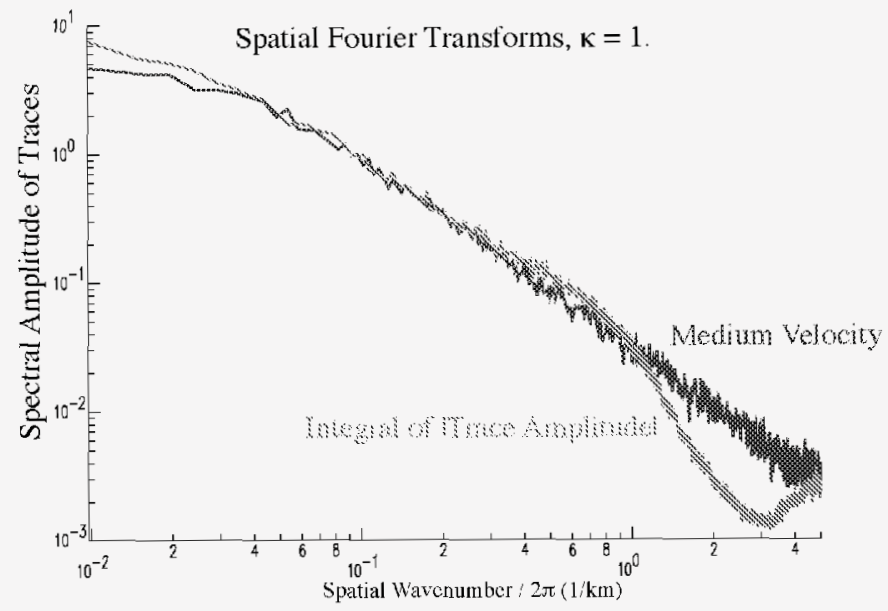

Figure 2. Comparison of the spectrum of the spatial fluctuation of medium velocity, shown in red, with the spectrum of the spatial fluctuation in integrated trace amplitude measured along a receiver array. 
Figure 3 shows an example of applying the method to simulated AVO data for a homogeneous half space over a heterogeneous half space. In this case, the wavenumber spectrum of the velocity heterogeneity has a shape that is similar to that of the spectrum of the integrated trace amplitude measured on the earth's surface. This means that we can obtain estimates of the spatial variation in medium heterogeneity from trace data. We have found that the method we have developed for characterizing the spatial velocity fluctuation is also applicable to inferring spectrum of fracture spacing (Pearce et al., 2003).

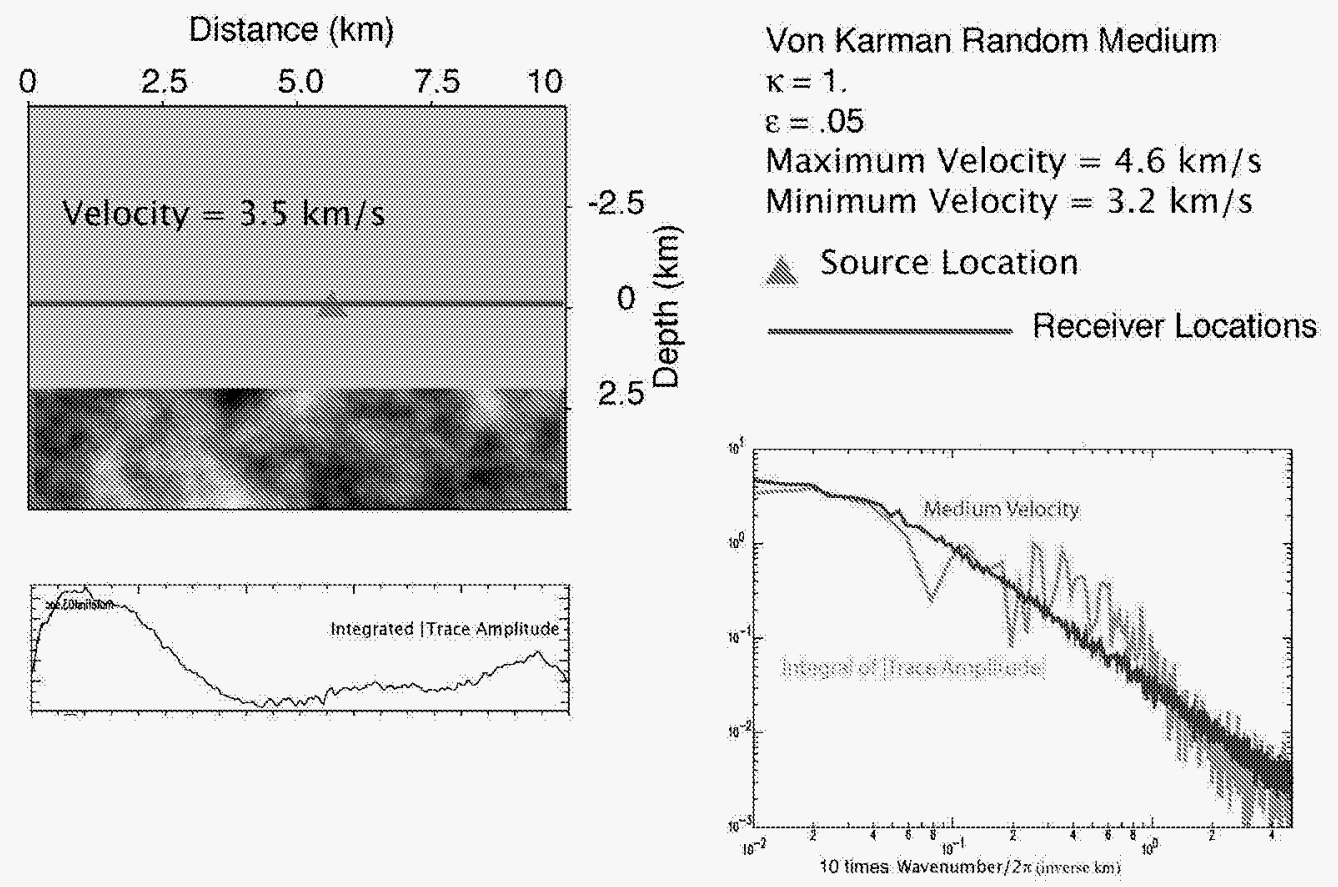

Figure 3. Upper left: model of a homogeneous half space in contact with a heterogeneous half space where the heterogeneity is defined using a von Kármán autocorrelation function (Sato and Fehler, 1998). Source wavelet is a $20 \mathrm{~Hz}$ Ricker-type source. Wavefield is recorded by an array of receivers located along the green line. At each trace location, the absolute value of the trace amplitude is integrated and the spatial variation of the result is shown in the lower left-hand figure. The spatial spectrum of the integral of the absolute value of the trace amplitude has a spatial spectrum that is similar to that of the spatial spectrum of the velocity heterogeneity of the lower half space as shown in the lower right-hand graph.

\subsection{Frequency-Dependent Reflectivity}

Since wave equation downward continuation is carried out using a wave-theory based method, the frequency dependent information about the amplitude and phase (the spectra) of the wavefield is preserved. Therefore, we can analyze not only the amplitudes of the reflected waves but also their phases for different frequencies, or in other words, all attributes of the reflected waves. To show the usefulness of the frequency-dependent 
reflectivity, we developed a method for calculating the theoretical frequency-dependent AVO responses for reservoir structures having different $Q$ models. The frequency characteristics of intrinsic attenuation have significant effects on AVO responses. Figure 4a shows frequency-dependent $\mathrm{Q}$ models where Q-1 $=f(a+b f 2), f$ is frequency, and $a$ and $b$ are two constants. Figure $4 \mathrm{~b}$ shows the reflectivity from an elastic oil-sand interface versus frequency and offset. A fre quency-dependent $\mathrm{Q}$ with $a=100$ and $b=0.1$ is used in the calculation. Figure $4 \mathrm{c}$ shows the residual reflectivity (by subtracting the reflectivity from a pure elastic model). Figure $4 \mathrm{~d}$ shows a similar result to Figure $4 \mathrm{c}$ except a different Q model with $a=40$ and $b=0.4$ is used. We see that the local reflections for models with frequency-dependent Qs may generate amplitude-offset relations that are different from those for models with frequency-independent Qs.
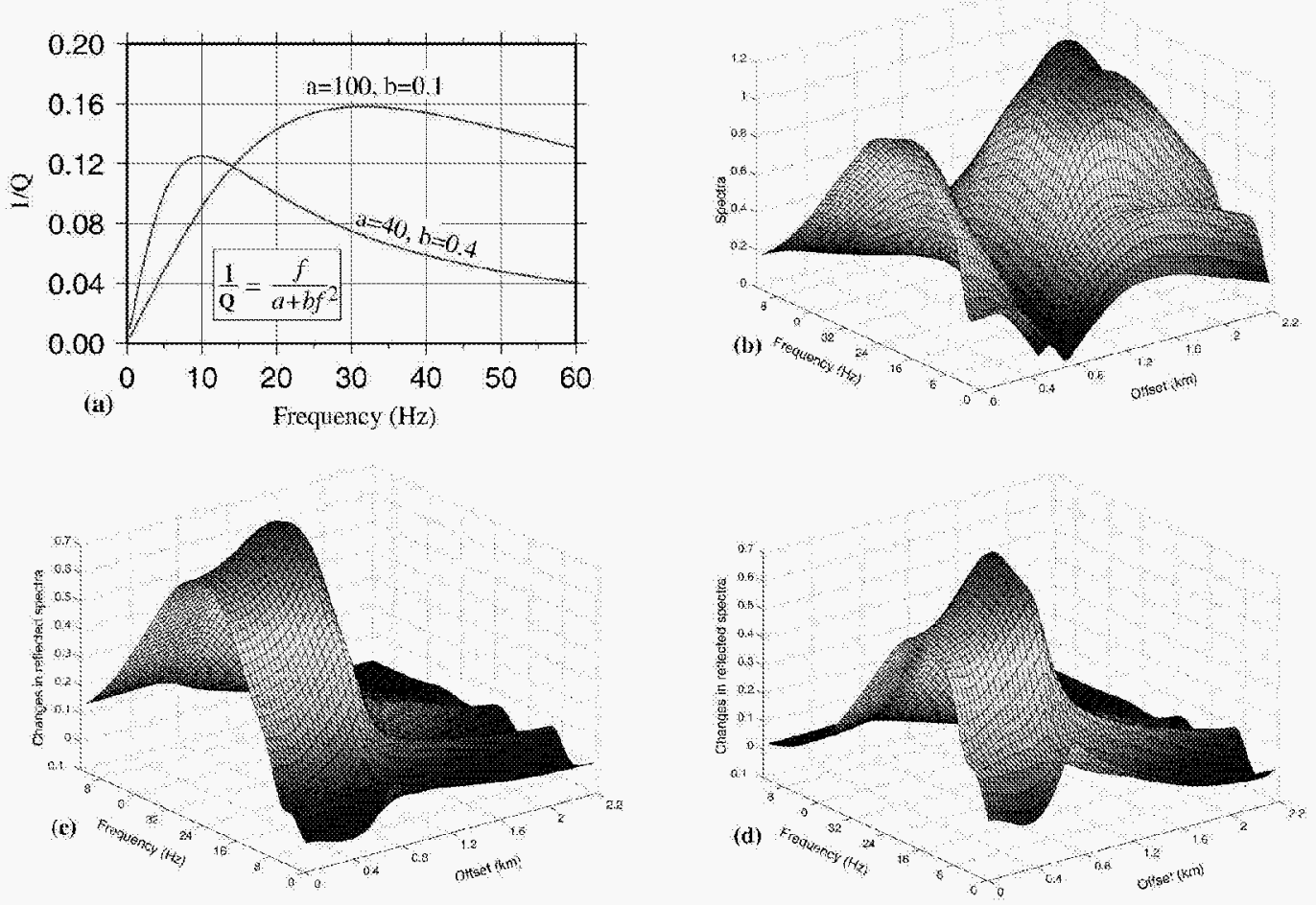

Figure 4. (a) Frequency-dependent Q models. (b) Reflectivity from an oil/sand interface as a function of frequency and offset. A frequency-dependent $\mathrm{Q}$ model with $\mathrm{a}=100$ and $\mathrm{b}=0.1$ is used. (c) Residual reflectivity by subtracting the reflectivity of a pure elastic model from (b). (d) Similar to (c) except a $Q$ model with $a=40$ and $b=0.4$ is used. 


\section{EXTRACTING LOCAL ANGLE DOMAIN INFORMATION FROM WAVEFIELD}

The recent developments of wave equation based migration methods (e.g., Stoffa et al., 1990; Ristow and Ruhl, 1994; Jin, et al., 1998; Xie and Wu, 1998; Huang and Fehler, 2000; Xie et al., 2000; Biondi, 2002) provided accurate propagators for seismic wave extrapolation. In addition to generate high quality subsurface images, these propagators also allow many analyses and inversions, which previously could only be carried on the surface, to be made at the depth using migrated wavefield. For example, the AVO analysis using offset information is traditionally applied to surface data. Now, the surface data can be extrapolated to the target area and then analysis can be made at the depth. The advantage of this technique is that ambiguities caused by the complicated propagation can be effectively eliminated. These analyses can be made by investigating migrated wavefield in different domains. The angle domain common image gather is often very useful for velocity updating and AVA analysis. Several robust approaches (Prucha, et. al., 1999; Mosher, 2001; Liu, et. al., 2001; Rickett and Sava, 2001) have been developed for this purpose. The target of this chapter has two fold. First, we propose a new approach to extract angle domain information from migrated wavefield. This method is based on a local plane wave analysis, which does not rely on a specific propagator such as those in common-offset domain or offset plane wave domain. It can be applied to almost any wave propagation method. Second, we propose further analysis on angle related information based on the local image matrix concept. Useful information about the reflectors can be obtained by properly sorting the energy in the image matrix. Numerical tests using simple models and the 2D SEG/EAGE Salt model are conducted to demonstrate this method.

\subsection{Angle Domain Analysis for Migrated Wavefields}

A basic scattering/reflection experiment can be obtained by illuminating the target using an incident wave in direction $\theta^{I}$ and observe the scattered energy leaving the reflector in direction $\theta^{R}$. Figure 1 is a cartoon showing this process. Collecting all possible illumination/reflection directions forms an image matrix $I\left(\theta^{I}, \theta^{R}\right)$, which is the partial image as a function of incident and scattering angles.

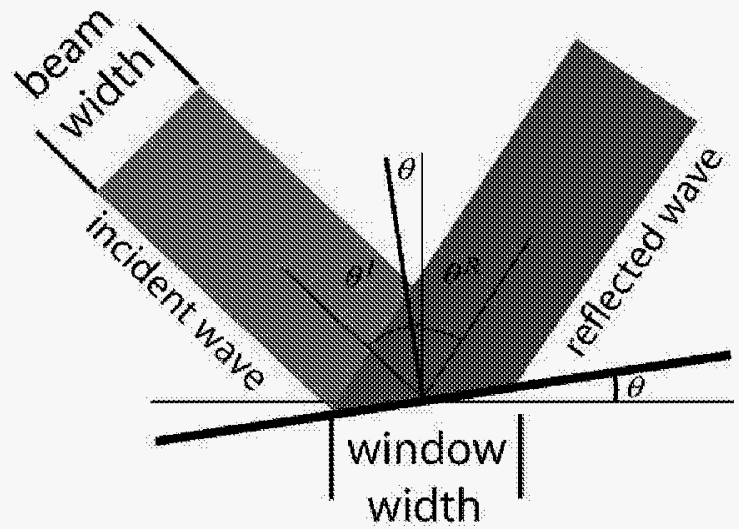

Figure 1. A basic reflection/scattering observation. 
Within the seismic migration process, the down going wavefield from the source and the upper going wavefield from receivers are both downward extrapolated. The angle related analysis can be made right at the target region. To decompose the wavefield into localized plane wave superposition and obtain the local image matrix, we use the windowed Fourier transform

$$
I\left(\mathbf{K}_{T}^{I}, \mathbf{K}_{T}^{R} ; \mathbf{x}_{T}, z, \omega\right)=C \cdot F\left[w\left(\mathbf{x}_{T}, \mathbf{x}_{T}^{\prime}\right) u^{I}\left(\mathbf{x}_{T}^{\prime}, z, \omega\right)\right] F\left[w\left(\mathbf{x}_{T}, \mathbf{x}_{T}^{\prime}\right) u^{R}\left(\mathbf{x}_{T}^{\prime}, z, \omega\right)\right]
$$

where $u^{\mathrm{I}}$ and $u^{\mathrm{R}}$ are incident and scattered waves, $w$ is a $2 \mathrm{D}$ moving window in the transverse space direction $\mathbf{x}_{T}^{\prime}$ and centered at $\mathbf{x}_{\mathrm{T}}$. A finite-sized window is required for localized wave number analysis under finite frequencies. The size of the window provides the trade off between the spatial and angle resolutions. $F[\cdot]$ is a $2 \mathrm{D}$ Fourier transform. $C$ is the normalization factor. The observation is centered at the horizontal position $\mathbf{x}_{\mathrm{T}}$ and depth $z$, and generated by an incident plane wave with local wavenumber $\mathbf{K}_{T}^{I}$ and a reflected plane wave with local wavenumber $\mathbf{K}_{T}^{R}$. These transverse wavenumbers can be transformed to angles via $\theta\left(K_{\mathrm{T}}\right)=\sin ^{-1}\left(K_{\mathrm{T}} / k\right)$, with $k=\omega / \alpha$ as the local wavenumber and $\alpha$ as the local velocity. The result can be summed up over frequency $\omega$. Similarly, the local image matrix can be calculated using a beam forming (slant stack) method directly in the space domain.

$$
\begin{aligned}
I\left(\theta^{I}, \theta^{R} ; \mathbf{x}_{T}, z, \omega\right) \\
=C \cdot \sum_{x_{T}^{\prime}}\left[w\left(\mathbf{x}_{T}, \mathbf{x}_{T}^{\prime}\right) u^{I}\left(\mathbf{x}_{T}^{\prime}, z, \omega\right) \exp \left[i k\left(\mathbf{x}_{T}^{\prime}-\mathbf{x}_{T}\right) \sin \theta^{I}\right]\right. \\
\quad \times \sum_{x_{T}^{\prime}}\left[w\left(\mathbf{x}_{T}, \mathbf{x}_{T}^{\prime}\right) u^{R}\left(\mathbf{x}_{T}^{\prime}, z, \omega\right) \exp \left[i k\left(\mathbf{x}_{T}^{\prime}-\mathbf{x}_{T}\right) \sin \theta^{R}\right]\right.
\end{aligned}
$$

For some wave equation based migration methods, e.g., the common offset migration, offset plane wave migration (Mosher, et al., 1996; Mosher and Foster, 1998; Jin and Wu, 1999; Prucha, et. al., 1999; Jin et al. 2000; Rickett and Sava, 2001) and wavelet transform based method (Wu et. al., 2000; Wu and Chen, 2001), their propagators intrinsically carry the angle information. The local scattering matrix can be easily constructed using this information.

Figure 2 shows features of the local image matrixes for some typical reflectors. The left panels are velocity models of different reflectors, and on the right are image matrixes with their horizontal and vertical axes as incident and scattering angles. The local image matrixes are obtained at the center of the model using a 32 grid space window. For a locally horizontal reflector (A), the energy is concentrated at the diagonal of the matrix, which meets the reflection principle, i.e., incident angle equals the reflection angle. For a dipping reflector (B), the energy still forms a strip parallel to the diagonal but shifts for a distance. If the reflector is more complicated than a flat interface, for example a curved interface (C), uneven rough interface (D), random layers (E), etc., their image matrixes will appear to be more complicated.

Depending on analysis purposes, the energy in the image matrix can be sorted in different ways. Figure 3 is a sketch showing possible analyses. The horizontal and vertical axes denote incidence and scattering angles. Investigating energy distribution 
along the diagonal direction (from upper-left to lower-right) gives angle related information that can be used in velocity updating, AVA analysis etc. For a dipping reflector, its energy will be shifted form the main diagonal. The offset of the energy measured along the upper-right to lower-left direction carries the dipping information of the structure. Other useful image gathers may also be constructed by properly summing up the energy. For example, integrating the energy along the horizontal direction gives the common scattering angle gather, and summing up the energy along the vertical direction gives the common illumination angle gather, etc.
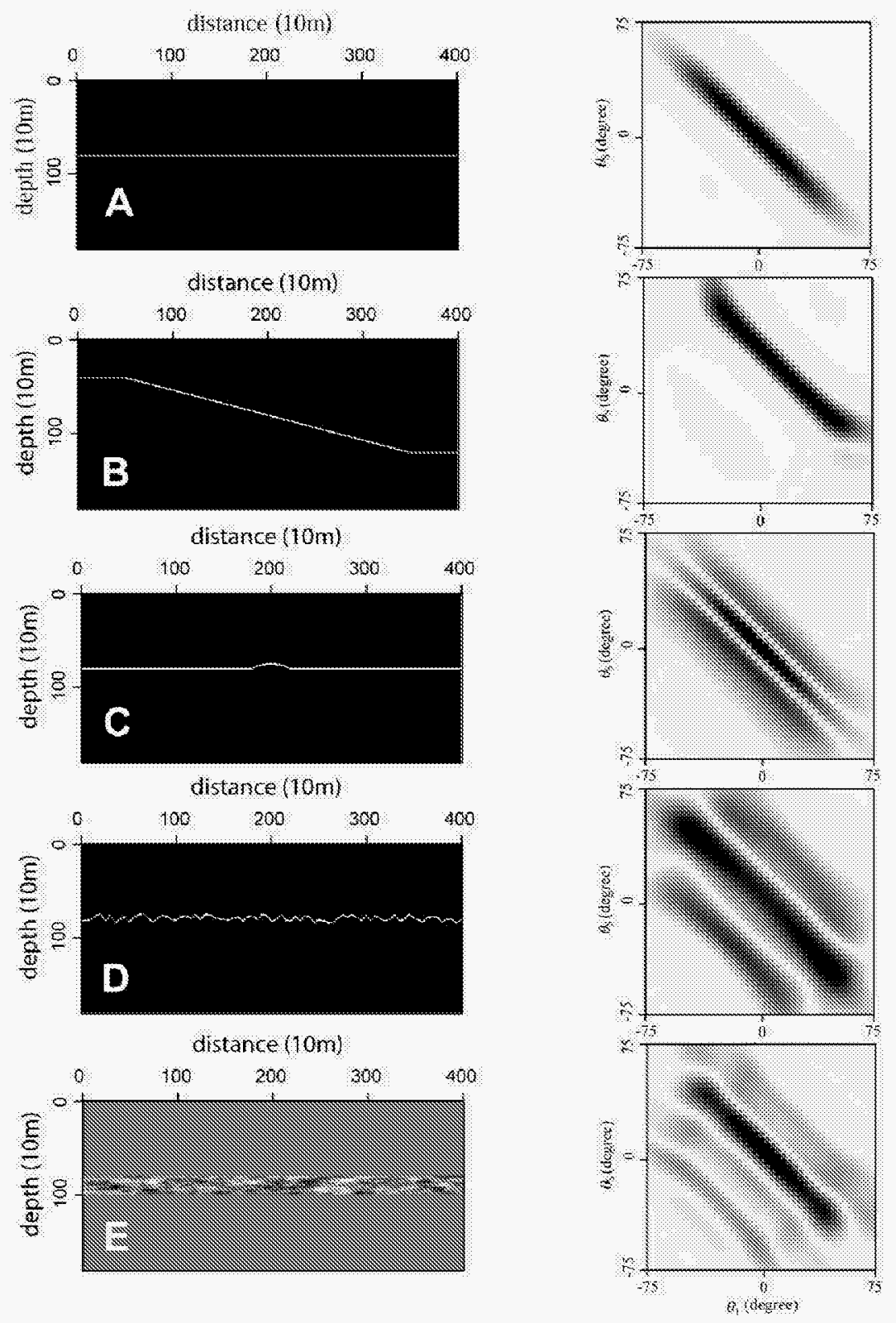

Figure 2. The local image matrixes for typical structures. On the left are velocity models and on the right are image matrixes. From upper to bottom are (A) a locally horizontal reflector, (B) a dipping reflector, (c) a curved interface, (D) an uneven rough interface, and (E) a random layer. 


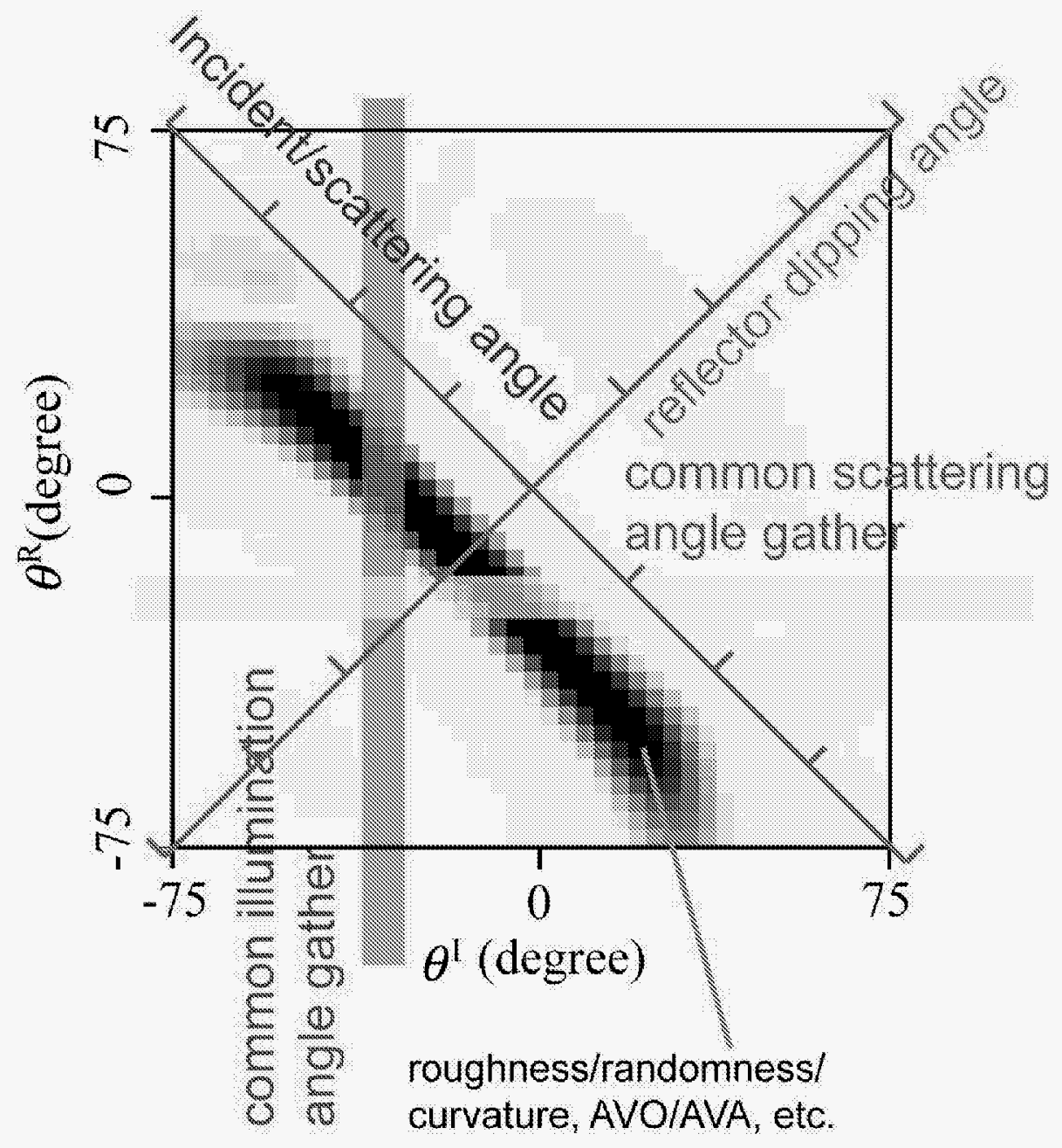

Figure 3. Cartoon showing the local image matrix and various types of analyses can be made.

\subsection{Numerical Examples}

To show the applications of the angle domain analysis, we use a simple 2D model. The velocity model is shown in Figure 4A. It is composed of a $3.5 \mathrm{~km} / \mathrm{s}$ constant background and four $4.0 \mathrm{~km} / \mathrm{s}$ reflectors. Sixty shots on the surface are used to illuminate the model and a wide-angle generalized screen propagator (Xie and $\mathrm{Wu}, 1998$; Xie et. al. 2000) is used for the wavefield extrapolation. The migrated image is shown in Figure 4B. The angle information is calculated at horizontal locations 50,100, 150 and 200, respectively. Figure $4 \mathrm{C}$ gives the angle domain image gathers using the diagonal energy only. As expected, image gathers reflect horizontal structures very well but do not reflect the dipping structures. In figure $4 \mathrm{D}$ the energy in the image matrix is summed along the upper-right to lower-left direction. With the off diagonal energy add in, the angle image gathers for the dipping part of the structure are properly produced. Normal incidences are automatically aligned on the center line. Due to the illumination condition, the angle coverage decreases with the depth. These angle image gathers can be used as the basis for 
velocity updating and AVA analysis. As has been mentioned above, the shift of energy distribution from the diagonal gives the measurement of the dip angle. By summing up the energy along the upper-left to lower-right direction, the energy distribution along upper-right to lower-left direction (refer to the cartoon in Figure 3) gives the information about reflector dips. Shown in Figure 4E are measurements of dipping angles. At distances 50 and 200, where the reflectors are flat, all energy peaks are centered at diagonals. For profiles at distances 100 and 150, the first reflector is flat but followed by three reflectors with increasing dipping angles. The shift of energy peaks from the center lines reveals their dip angles which can be quantitatively measured.

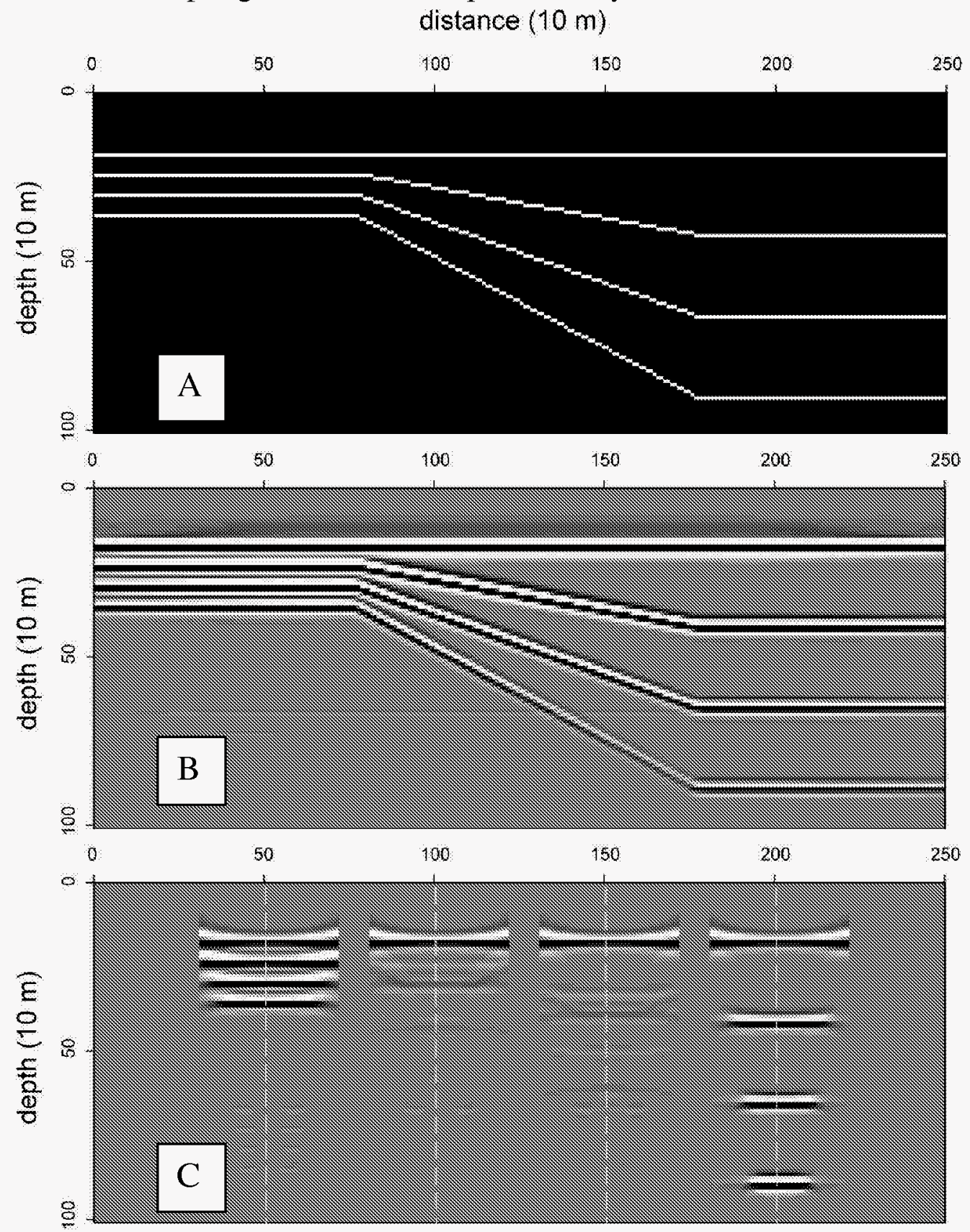



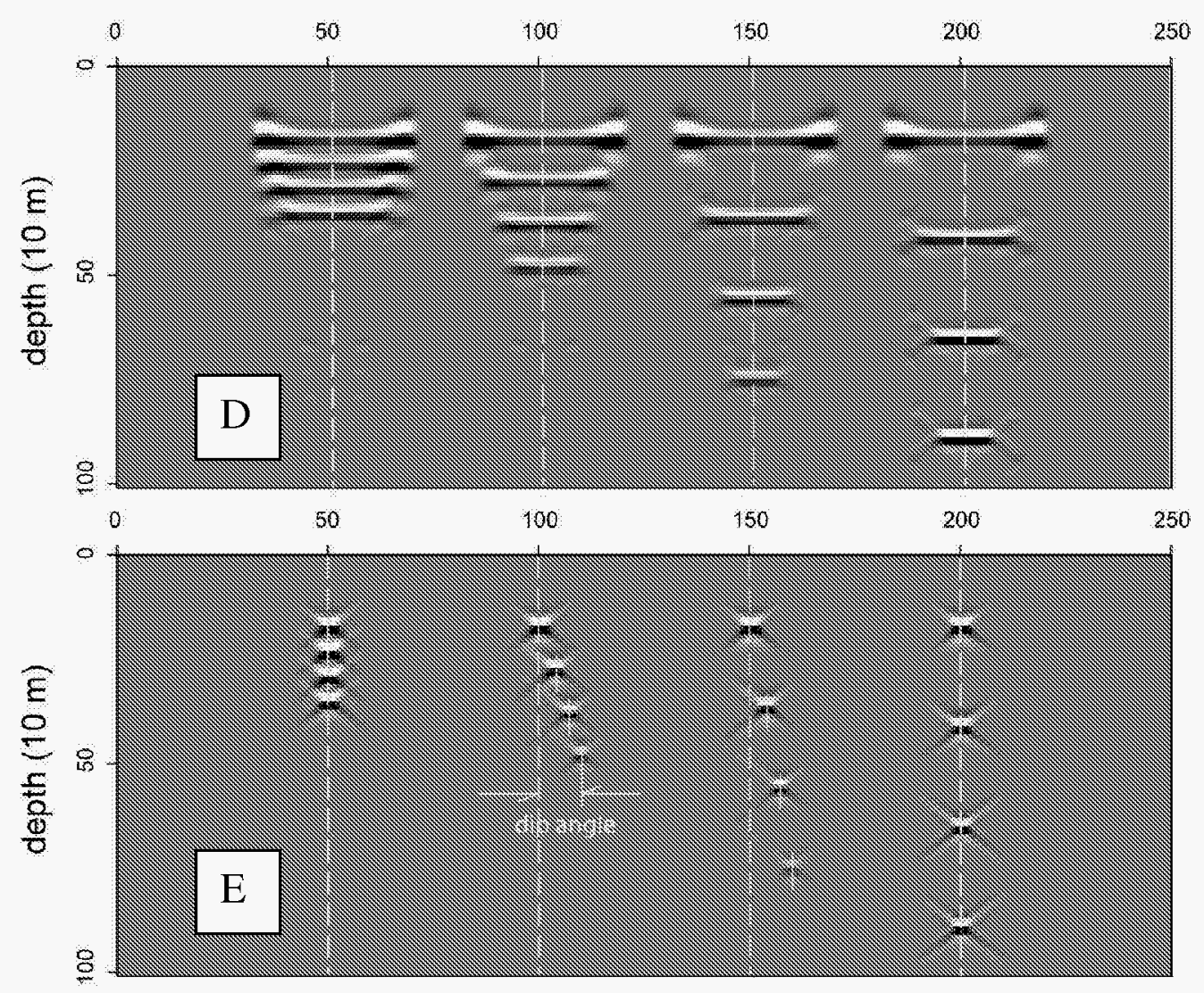

Figure 4. A simple numerical example. For details see the text.

The angle domain common image gather provide a powerful tool for velocity updating. Figure 5 shows the comparison of the sensitivity of angle domain common image gathers to errors in velocity models. The upper panel gives the same velocity model used for previous examples. The middle panel shows angle domain common image gathers calculated for a velocity model in which a $-10 \%$ negative velocity error is purposely added to layers 2 to 4 . A prominent feature is that the entire image is shifted upward except for the uppermost reflector. The angle image for the uppermost reflector is still flat since the first layer has a correct velocity. For the rest of the reflectors, their wide-angle images are bended toward the shallower depth, which result from wide-angle waves passed a longer distance before they reached the target region. The lower panel shows angle domain common image gathers calculated for a velocity model which has $+10 \%$ velocity error in layers 2 to 4 . The entire image, except for the first reflector, is shifted downward with wide-angle images bended toward the deeper depth. The angle related information shown in this example can be directly used for velocity updating. 


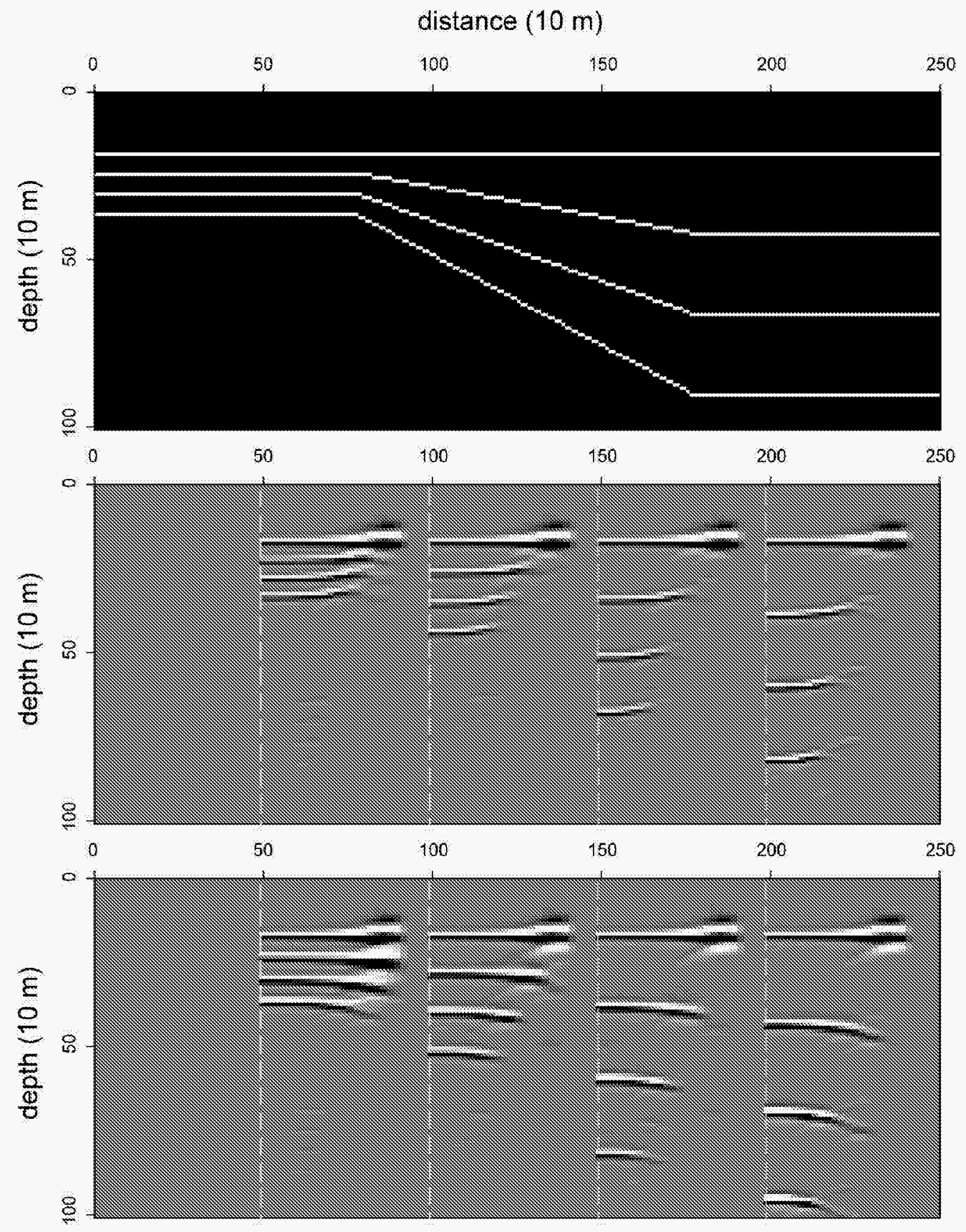

Figure 5. Comparison of angle domain common image gathers calculated for velocity models with errors. The upper panel gives the same velocity model used for the previous example. The middle panel shows angle domain common image gathers calculated for a velocity model which has a $-10 \%$ negative velocity error in layers 2 to 4 . The lower panel shows angle domain common image gathers calculated for a velocity model which has a $+10 \%$ velocity error in layers 2 to 4 . 


\section{ILLUMINATION ANALYSIS IN THE LOCAL ANGLE-DOMAIN}

Advances in seismic technology, particularly in prestack depth migration, have made significant improvement in providing reliable high-resolution images for complex structures. However, there is still a need to better understand how various factors affect image qualities. The seismic illumination analysis is a useful estimation that gives potential detecting power of a specific acquisition system for a given structure. Traditionally, illumination analysis is based on the ray tracing technique (Schneider, 1999; Bear et al., 2000; Muerdter et al., 2001abc). The ray-based methods can provide both the intensity and direction information carried in the wavefield. However, the high frequency asymptotic approximation and the singularity problem of the ray theory may severely limit its accuracy in complex regions (Hoffmann, 2001). Full-wave finitedifference method is widely used for wave propagation simulation. However, for illumination analysis purpose, it usually provides only the total illumination. The directional information of the wave propagation is lost. Finite-difference simulation is also expensive, which prohibits it from practical application for illumination analysis.

The recently developed dual-domain one-way wave equation based methods (e.g., Stoffa et al., 1990; Ristow and Ruhl, 1994; Jin, et al., 1998, 2002; Xie and Wu, 1998; Huang and Fehler, 2000; Xie et al., 2000; Wu and Chen, 2001; Biondi, 2002) provided accurate propagators for seismic wave extrapolation. To apply these propagators to illumination analysis, the central part is extracting angle related information from the propagated wavefield. To do this, $\mathrm{Wu}$ and Chen (2002) used the wavelet transform theory to decompose the wavefield into beamlets, which are localized in both space and directions, and successfully applied it to the illumination analysis. Xie and $\mathrm{Wu}$ (2002) proposed another approach based on the local plane wave analysis. In this research, we developed an illumination analysis method based on generalized screen propagator (Jin, et al., 1998; $\mathrm{Xie}$ and $\mathrm{Wu}, 1998$ ) and the local plane wave analysis (Xie and $\mathrm{Wu}, 2002$ ). Using this method, the seismic energy from the sources and receivers are propagated to the target region with a one-way wave equation based propagator. Then, a local plane wave analysis is conducted and directional energy fluxes are obtained from wavefields. By properly sorting energy fluxes in the target region, the total illumination, directional illumination, total dip-response and directional dip-response can be obtained.

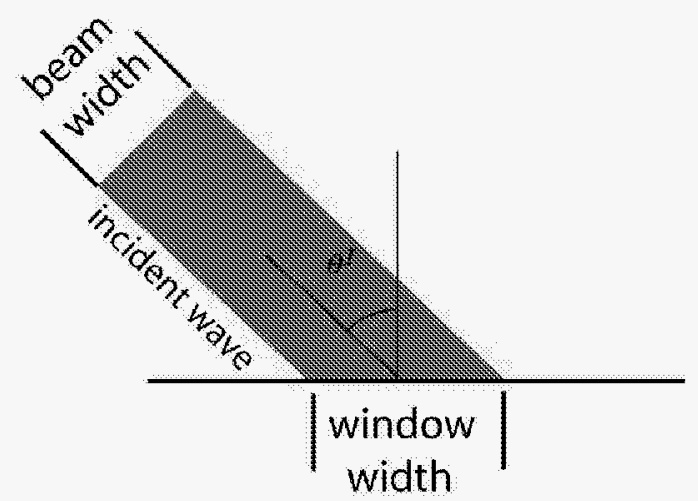

Figure 1. Basic angle domain information. 


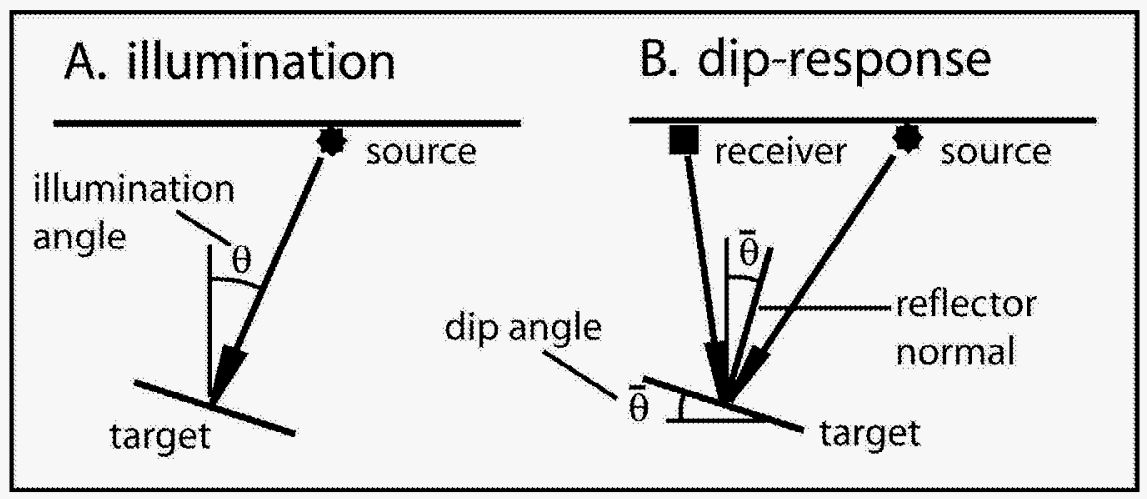

Figure 2. A sketch showing (a) the directional illumination and (b) the acquisition dipresponse.

In the following sections, we first give a brief description on how to calculate the directional energy fluxes from the wavefield and apply them to the illumination analysis. Then, to demonstrate the potential applications of this method, illumination examples are calculated using the 3D SEG/EAGE Salt model. The results from the illumination analysis are compared with the prestack depth image obtained from the same model.

\subsection{Angle Domain Illumination analysis and dip-response analysis}

$\mathrm{Xie}$ and $\mathrm{Wu}$ (2002) proposed an approach to extract angle related information from the wavefield using a local plane wave analysis. Figure 1 is a sketch showing the geometry of angle domain analysis. Using this method, the seismic energy from the sources or receivers is propagated to the target region with a wave equation based propagator. Then, a windowed Fourier transform is used to decompose the wavefield into localized plane waves

$$
u\left(\mathbf{r}_{S} ; \mathbf{x}_{T}, z, \mathbf{K}_{T}, \omega\right)=F\left[w\left(\mathbf{x}_{T}, \mathbf{x}_{T}^{\prime}\right) u\left(\mathbf{r}_{S} ; \mathbf{x}_{T}^{\prime}, z, \omega\right)\right],
$$

where $u\left(\mathbf{r}_{S} ; \mathbf{x}_{T}^{\prime}, z, \omega\right)$ is the incoming wave, $\mathbf{r}=\left(\mathbf{x}_{T}, z\right)$ is the location, $\mathbf{x}_{\mathrm{T}}$ is the horizontal location, $z$ is the depth, $\mathbf{r}_{\mathrm{S}}$ is the source location, $u\left(\mathbf{r}_{S} ; \mathbf{x}_{T}, z, \mathbf{K}_{T}, \omega\right)$ is the local plane wave, $w$ is a $2 \mathrm{D}$ window in the horizontal direction $\mathbf{x}_{T}^{\prime}$ and centered at $\mathbf{x}_{\mathrm{T}}, \mathbf{K}_{\mathrm{T}}$ is the local horizontal wavenumber, $F[\cdot]$ is a $2 \mathrm{D}$ Fourier transform. A finite-sized window is required for local plane wave analysis under finite frequencies. The size of the window provides the trade off between the spatial and angle resolutions. The local horizontal wavenumber $\mathbf{K}_{\mathrm{T}}$ can be transformed to angle $\boldsymbol{\theta}=(\theta, \varphi)$, where $\theta\left(\mathbf{K}_{\mathrm{T}}\right)=\sin ^{-1}\left(K_{\mathrm{T}} / k\right)$ and $\varphi\left(\mathbf{K}_{\mathrm{T}}\right)=\tan ^{-}$ ${ }^{1}\left(K_{\mathrm{Y}} / K_{\mathrm{X}}\right), k=\omega / \alpha$ is the local wavenumber and $\alpha$ is the local velocity. The local plane wave can also be written as $u\left(\mathbf{r}_{S} ; \mathbf{x}_{T}, z, \boldsymbol{\theta}, \omega\right)$.

An illumination is the energy flux at the target generated by the seismic source (see Figure 2a). The illumination can be described with the intensity and direction of the incoming energy

$$
I\left(\mathbf{r}_{S} ; \mathbf{x}_{T}, z, \mathbf{\theta}, \omega\right)=u\left(\mathbf{r}_{S} ; \mathbf{x}_{T}, z, \boldsymbol{\theta}, \omega\right) \cdot u^{*}\left(\mathbf{r}_{S} ; \mathbf{x}_{T}, z, \mathbf{\theta}, \omega\right)
$$


where $I$ is called directional illumination (DI), “*” denotes complex conjugate. For an acquisition system composed of multiple sources, their combined illumination can be obtained by summing up energy from the individual sources

\section{Distance $(20 \mathrm{~m})$}

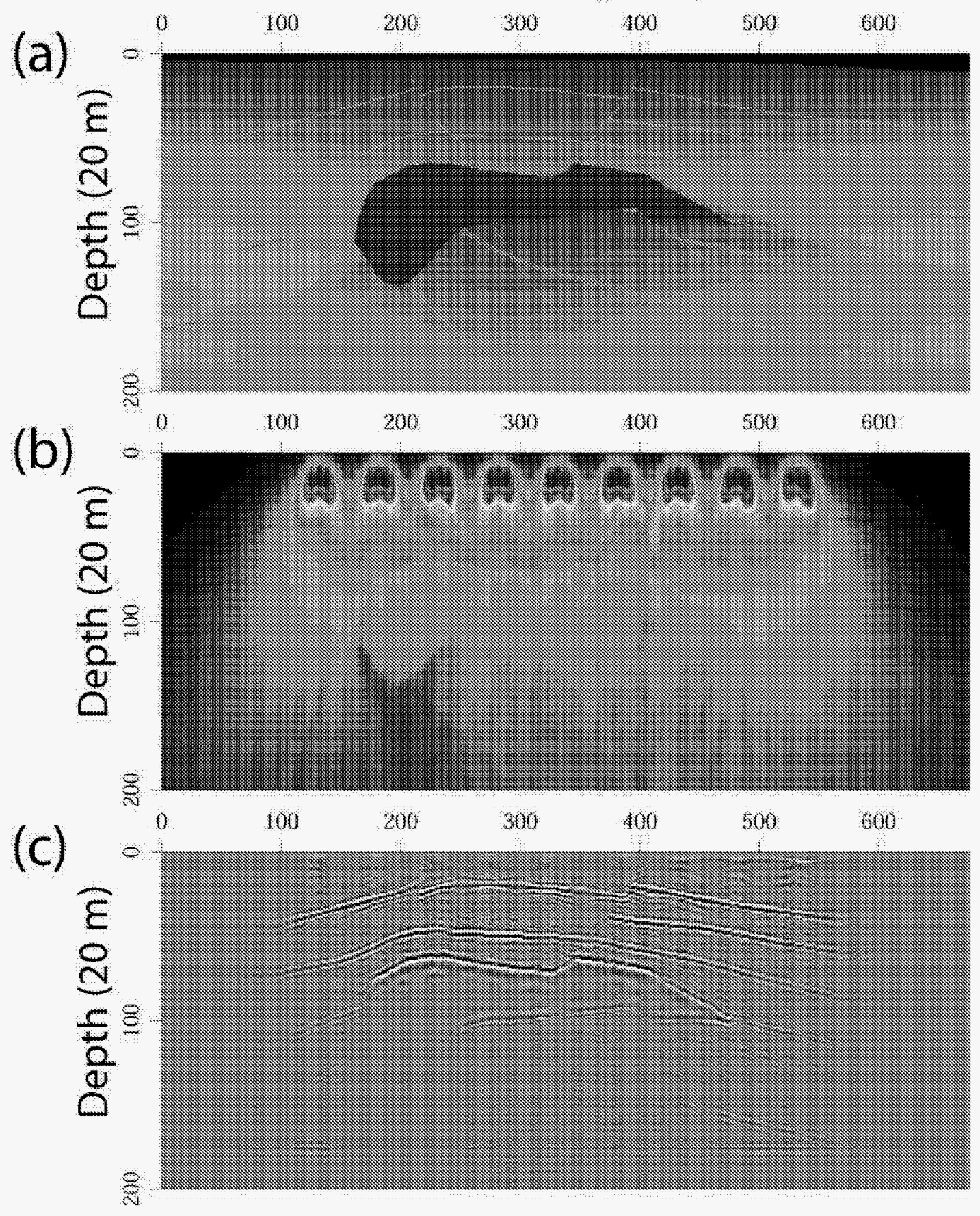

Figure 3. Comparison between the velocity model, illumination map and prestack depth image for the SEG/EAGE 45 shot data set. Shown in the figure is a cross section at $x=$ $6000 \mathrm{~m}$, where (a) is the velocity model, (b) is the total illumination map, and (c) is the depth image. 


$$
I\left(\mathbf{x}_{T}, z, \boldsymbol{\theta}, \omega\right)=\sum_{\mathbf{r}_{S}} I\left(\mathbf{r}_{S} ; \mathbf{x}_{T}, z, \boldsymbol{\theta}, \omega\right) .
$$

DI can be further sorted according to different purposes to give useful information. For example, the total illumination can be obtained by summing up energy fluxes from all directions. The result can also be summed up over the frequency $\omega$.

The acquisition dip-response (ADR) is a simulation of the detecting power of a sourcereceiver pair (see Figure 1B), which can be expressed as

$$
R\left(\mathbf{r}_{S} ; \mathbf{r}_{R} ; \mathbf{x}_{T}, z, \overline{\boldsymbol{\theta}}, \omega\right)=\left[I\left(\mathbf{r}_{S} ; \mathbf{x}_{T}, z, \boldsymbol{\theta}_{S}, \omega\right) \cdot I\left(\mathbf{r}_{R} ; \mathbf{x}_{T}, z, \boldsymbol{\theta}_{R}, \omega\right)\right]^{1 / 2},
$$

where $\mathbf{r}_{\mathrm{R}}$ is the location of the receiver, $I\left(\mathbf{r}_{\mathrm{S}}\right)$ and $I\left(\mathbf{r}_{\mathrm{R}}\right)$ are DIs from the source and receiver, $\boldsymbol{\theta}_{\mathrm{S}}$ and $\boldsymbol{\theta}_{\mathrm{R}}$ are their illumination angles, $\overline{\boldsymbol{\theta}}$ is the angle bisector of $\boldsymbol{\theta}_{\mathrm{S}}$ and $\boldsymbol{\theta}_{\mathrm{R}}$, which is also the dipping angle of the favorite reflector. The energy fluxes are calculated from both source and receiver to the target. According to the seismic representation reciprocity, the later is equivalent to the up-going energy flux from the target to the receiver. Combining both energy fluxes together equivalents to a source-reflectorreceiver process with a unit reflectivity. Its strength gives the detecting power of a specific source-receiver pair to the target in case there is a reflector. The most favorite structures that can be detected are those with their normal vectors parallel to the angle bisector of source and receiver side incoming fluxes. For an acquisition system composed of multiple source-receiver pairs, its detecting power can be obtained by summing up contributions from individual source-receiver pairs according to the specific acquisition geometry, (e.g., multiple shots, cable length, sail direction, etc.)

$$
R\left(\mathbf{x}_{T}, z, \overline{\boldsymbol{\theta}}, \omega\right)=\sum_{\mathbf{r}_{S}} \sum_{\mathbf{r}_{R}} R\left(\mathbf{r}_{S} ; \mathbf{r}_{R} ; \mathbf{x}_{T}, z, \overline{\boldsymbol{\theta}}, \omega\right) \text {. }
$$

\subsection{Numerical Examples of Illumination Calculation}

To demonstrate the application of the illumination analysis, we calculated a number of numerical examples using the 3D SEG/EAGE salt velocity model. The first example simulates the illumination condition of the 45 shot data set. This data set represents a land type acquisition geometry. Figure 3 compared the velocity model, illumination map and prestack depth image for a cross section at $x=6000 \mathrm{~m}$, where (a) is the velocity model, (b) is the total illumination map, and (c) is the prestack depth image using the local cosine beamlet migration method (Luo and $\mathrm{Wu}, 2003$ ). The result clearly reveals that the poor illumination condition is responsible for the missing image under the left end of the salt body. Figure 4 is a similar result for a cross section at $x=8400 \mathrm{~m}$, where (a) is the velocity model, (b) is the vertical illumination map, and (c) is the depth image. Again we can see the correlations between illumination conditions and image qualities.

The second example simulates a marine type acquisition configuration. About 3500 surface shots are used to calculate the illumination in the model. Shown in Figure 5 are DI (directional illumination) maps in a cross section at $y=6100 \mathrm{~m}$, where (a) is the velocity model, (b) is the $45^{\circ}$ DI towards left, (c) is the vertical DI, and (d) is the $45^{\circ} \mathrm{DI}$ towards right. The results clearly show that the high velocity salt body effectively blocks 
the energy from reaching to the subsalt region. It is especially difficult for obliquely propagated energy to penetrate the salt body, which makes imaging the subsalt steep structures a challenge.

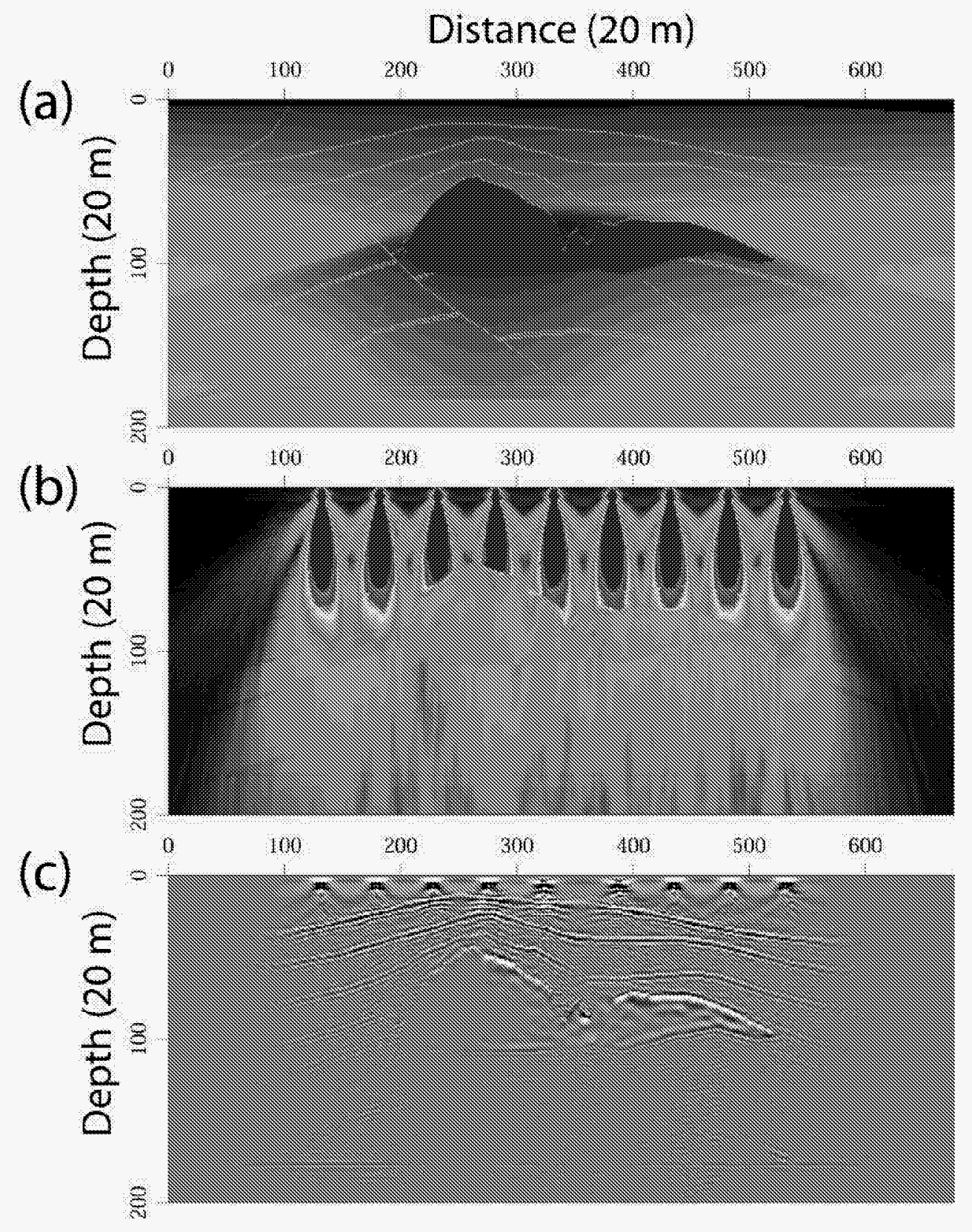

Figure 4. A similar result for the SEG/EAGE 45 shot data set. The cross section is located at $x=8400 \mathrm{~m}$. (a) is the velocity model, (b) is the vertical illumination map, and (c) is the depth image. 


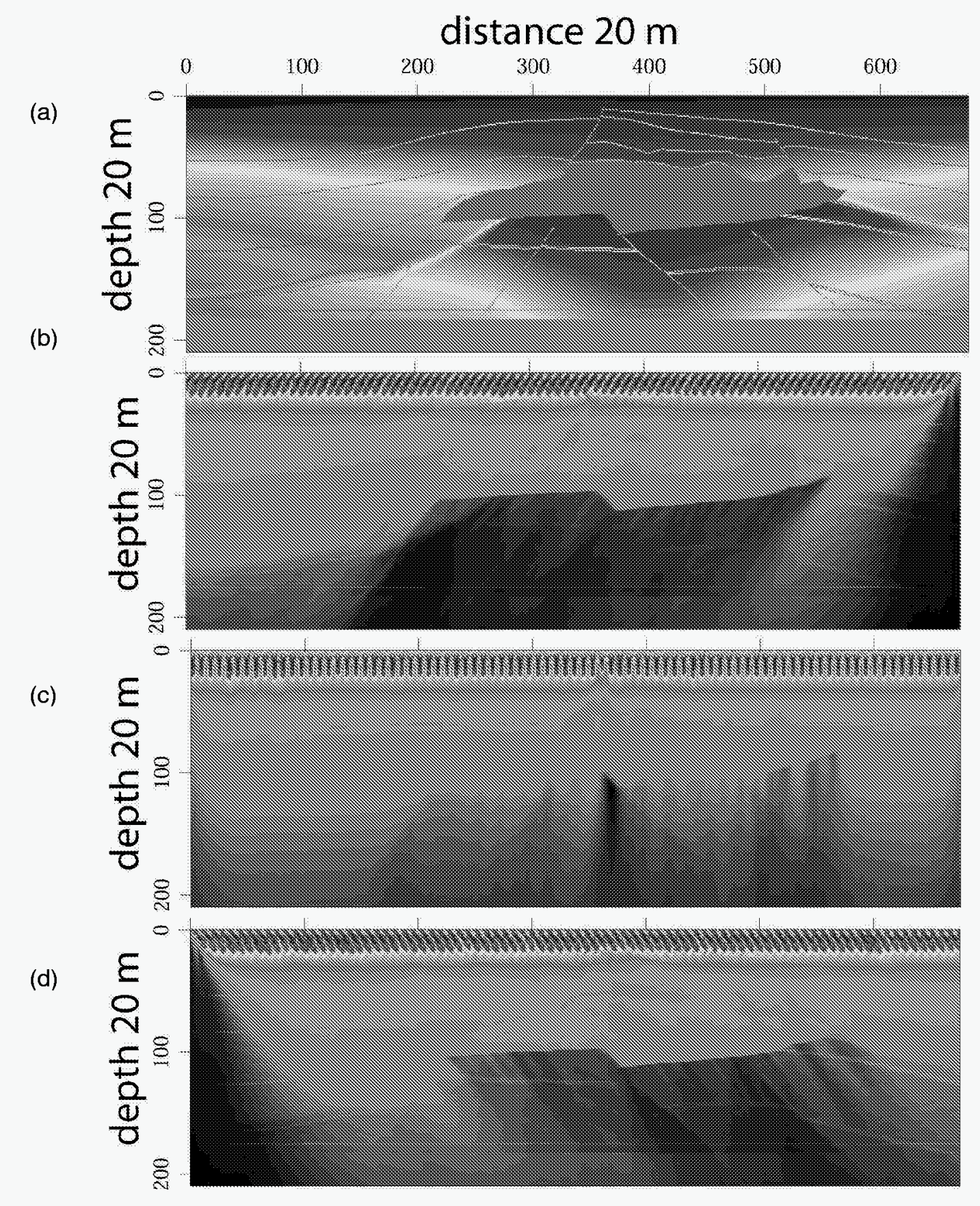

Figure 5. DI maps for the 3D SEG/EAGE salt model. Shown in the figure are cross sections at $\mathrm{y}=6100 \mathrm{~m}$, with (a) velocity model, (b) DI of $45^{\circ}$ left, (c) vertical DI, and (d) DI of $45^{\circ}$ right. 


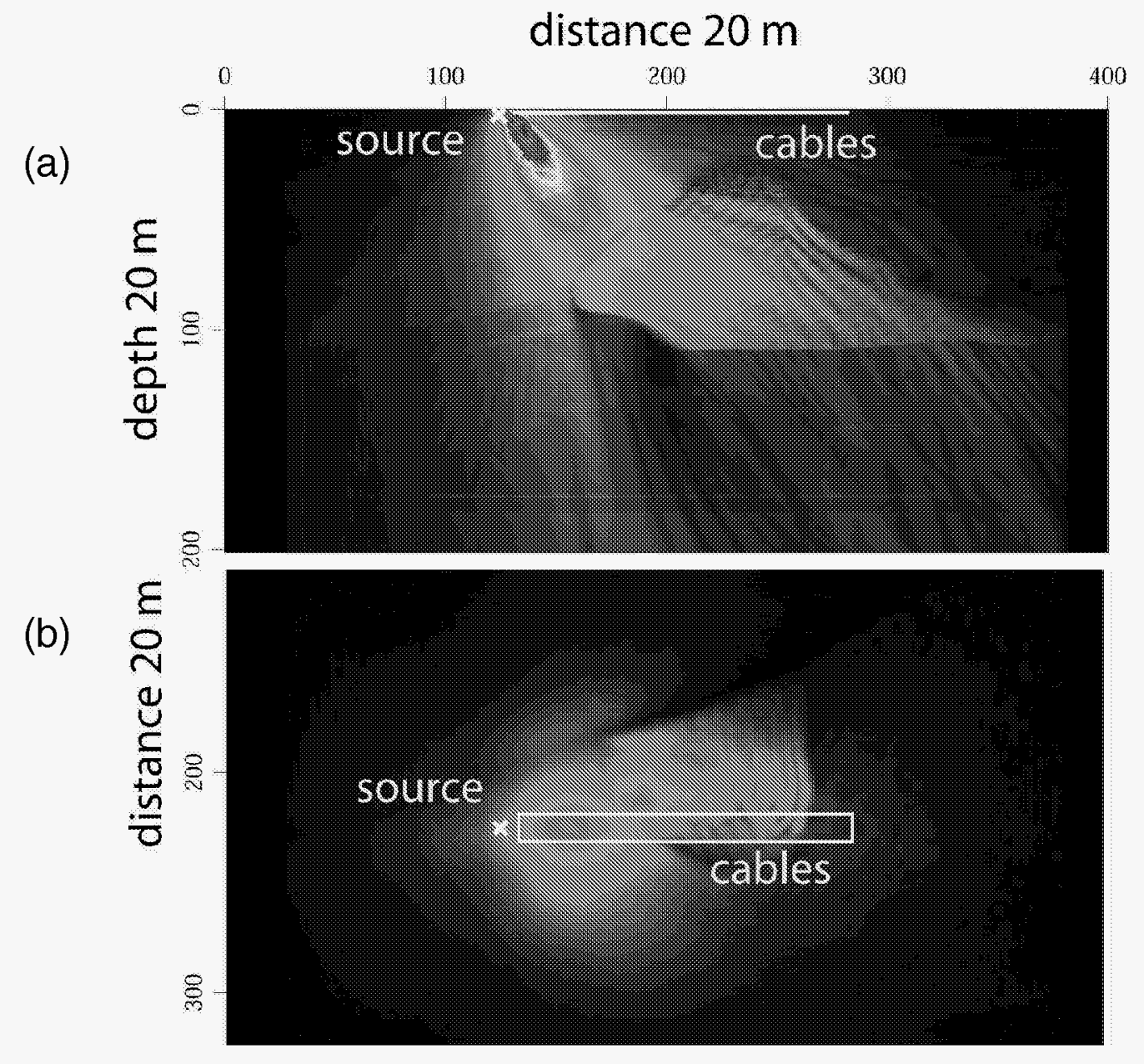

Figure 6. The ADR from a single shot in a 3D model. The dip angle is $30^{\circ}$. Shown in (a) is the vertical profile, (b) is the depth slice at $\mathrm{z}=1000 \mathrm{~m}$. The acquisition system composed of a source and four $3200 \mathrm{~m}$ cables, which have been marked in the figure.

The next example is the acquisition dip-response from a single shot and four $3200 \mathrm{~m}$ long cables in the 3D SEG/EAGE salt model. Shown in Figure 6 is the $30^{\circ}$ ADR (acquisition dip-response) in a cross section along the sail direction and in a depth slice at $z=1000 \mathrm{~m}$. The locations of the source and cables are indicated in the figure. These ADR maps reveal the relationship between the detecting power, the specific source-cable system and the complex velocity model. They can be viewed as the "Green's function" of the detecting power. The actual illumination condition in a prospecting area can be obtained by sum up the single shot ADRs according to the navigation and shooting condition. 


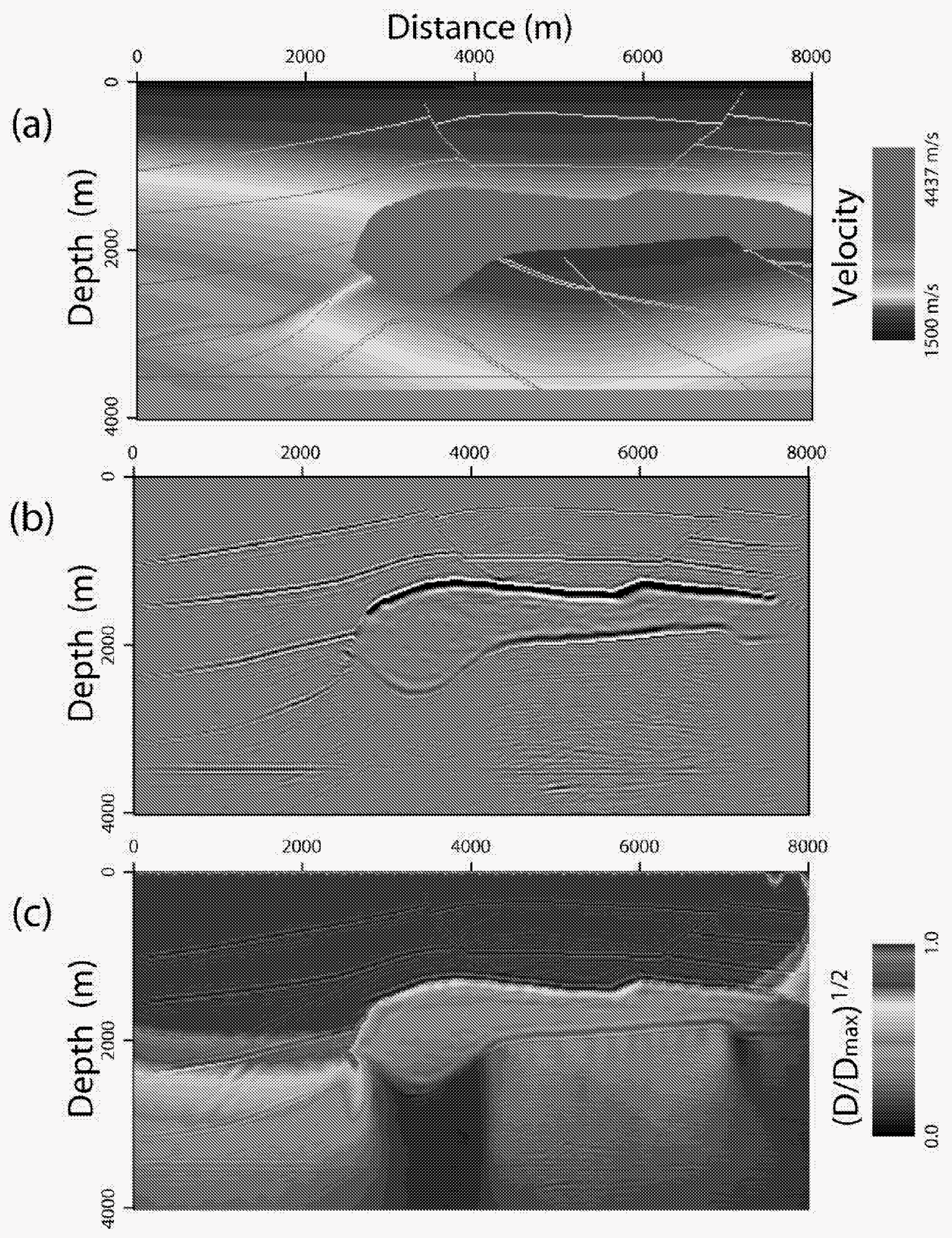

Figure 7. ADRs for the 3D SEG/EAGE salt model. Shown in the figure are cross sections at $y=2440 \mathrm{~m}$, with (a) velocity model, (b) prestack depth image and (c) vertical ADR. 


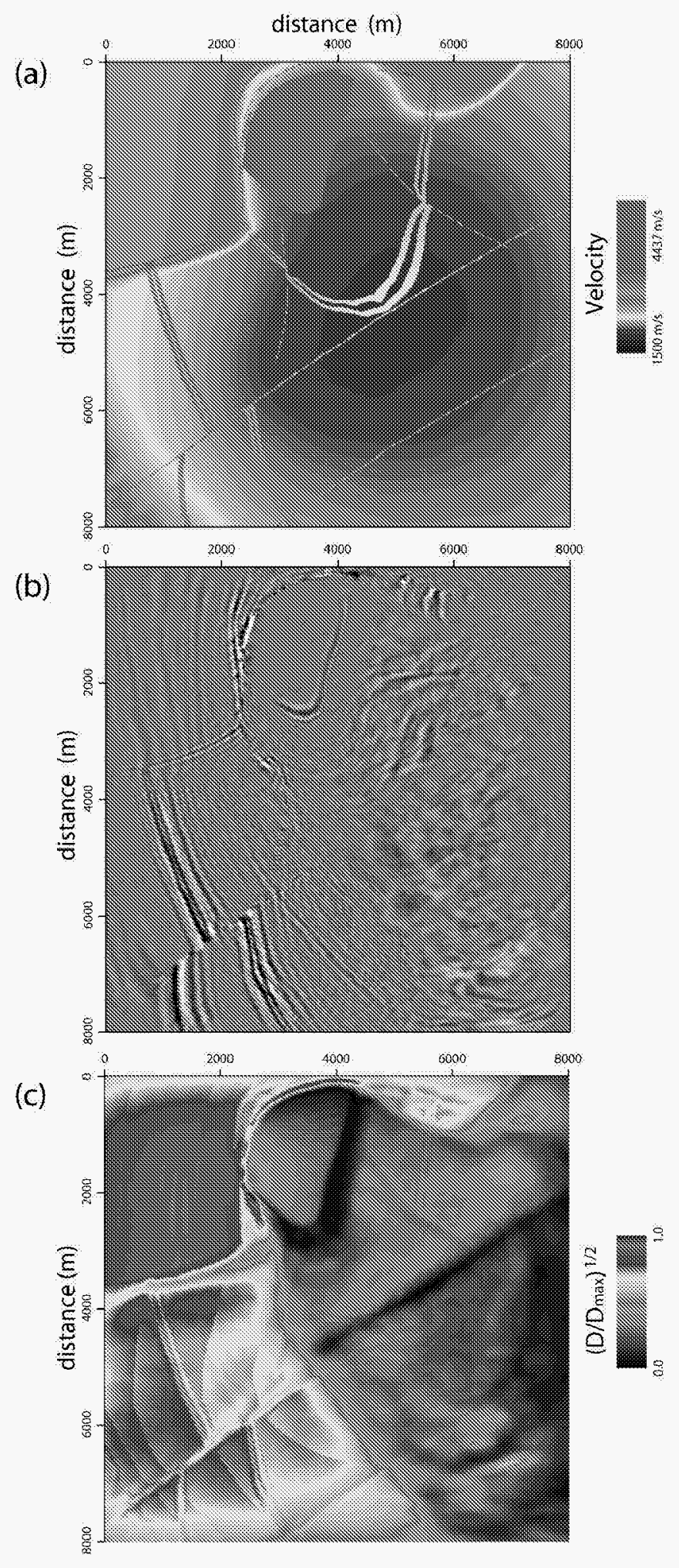

Figure 8. ADRs for the 3D SEG/EAGE salt model. Shown in the figure are depth slices at $\mathrm{z}=2500 \mathrm{~m}$, with (a) velocity model, (b) prestack depth image and (c) total ADR. 
The last example gives the acquisition dip-response, which simulates the narrow azimuth data set SEG/EAGE C3NA. About 500 surface shots are used to illuminate the model. Shown in Figure 7 is a cross section at $\mathrm{y}=2440 \mathrm{~m}$ (note the velocity model for C3NA is a subset from the SEG/EAGE velocity model), in which (a) is the velocity model, (b) is the prestack depth image using the common-offset method (Jin et al, 2002), and (c) is the vertical ADR map. In the ADR map, a shadow zone can be seen beneath the salt body. This shadow zone is consistent with the depth image where the subsalt structures, including the horizontal base line, are totally missing. Figure 8 gives similar result for the total ADR. Shown in the figure are depth slices at $z=2500 \mathrm{~m}$. In Figure 8b, the prestack depth image shows good quality on the left side, but the result is unsatisfied on the right side. The poor image quality can be explained with the low illumination region shown in Figure 8c.

\subsection{Acquisition Dip Response Analysis}

In the past, most techniques for calculating illumination intensity distributions were based on ray tracing modeling (Berkhout, 1997; Muerdter et al., 2001a; 2001b; 2001c; Schneider, 1999; Bear et al., 2000). The ray method can provide angle-dependent illumination at any point, which can be very important for understanding limitations of images and even for improving images. Although ray tracing is convenient and its results can be easily sorted into CRP (common reflection point) gathers, common offset gathers, or common reflection angle gathers to understand illumination attributes, the resulting illumination map may contain large errors due to the inherent high frequency approximation used in ray tracing. This may severely limit its accuracy (Hoffmann, 2001). On the other hand, finite difference (FD) modeling is a widely used method to do illumination analysis based on wave theory. However, FD is a space domain solution of the wave equation which can only provide the total illumination at any point and angledomain information is lost. In order to have reliable directional illumination, we need to have a wave-theory based method which provides reliable space and direction information. Advanced mathematical technologies, especially the newly developed wavelet transform and the more general frame theory, provide a solid foundation for the development of wavefield analysis in the local angle-domain. We have applied beamlet decomposition using the efficient decomposition schemes of Gabor-Daubechies frame (G-D frame) (Wu et al., 2000; Wu and Chen, 2001) to the wave field and derived the corresponding propagators in the eamlet domain that provides localizations in both space and direction (local wavenumber or angle) of the wave field, which makes it a natural tool to analyze directional illumination distributions and the aperture effects in the angledomain. In the following we show analysis of the directional aperture effects of the acquisition system for the 2D and 3D synthetic data sets of SEG/EAGE salt models (Wu and Chen, 2002, 2003; Xie et al., 2003). The acquisition aperture effects shown here are the ADR (acquisition dip-response) maps derived from the AAE (acquisition aperture efficacy) matrix. ADR gives the system response to local reflectors with different dipangles at each point in the image space. Figure 9 shows an album of ADR maps for the SEG/EAGE 2D salt model acquisition geometry. We see that the ADRs for different dipangles are quite different. Shadow zones for one dip-angle can be bright zones for other dip-angles. Therefore, to get a high-quality image that contains uniform illumination for 
structures of all dips, amplitude corrections must be done in the angle-domain. Figure 10 compares the prestack depth images with ADR (acquisition dip response) maps for the 2D SEG/EAGE salt model. Shown on the left are total illumination and total ADR; on the right are directional ADRs for horizontal reflectors and 45 degree dipping reflectors. We see that the total illumination and total ADR are not well related to the image quality. In contrast, the directional ADRs on the right show very good correlations to the reflectors with the corresponding dips. After obtaining the DI (directional illumination) and ADR, we can perform various amplitude corrections to improve the image quality. Figure 11 gives an example of amplitude correction. We see that after the preliminary correction based on illumination analysis, the image becomes stronger for the steeply-dipping faults located beneath the salt.
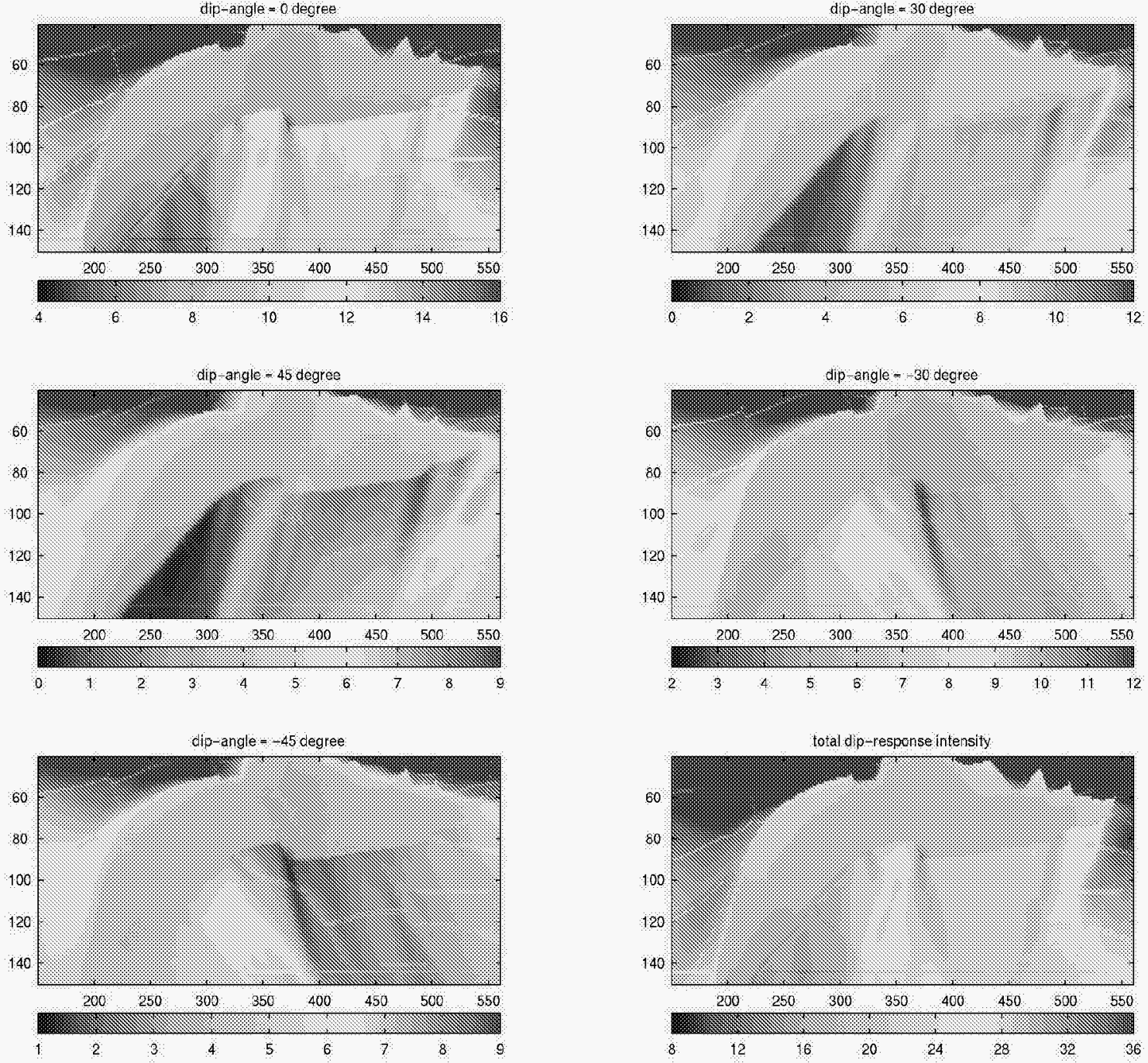

Figure 9. Acquisition-Dip-Response album of the 2D SEG-EAGE salt model from all the 325 shots with 176 receivers located to the left-side of each shot. Illumination angle is shown at the top of each plot. Angles are measured clockwise from vertical. 


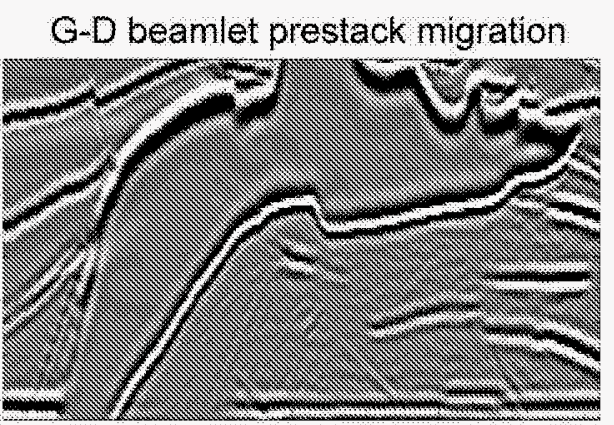

Total illumination

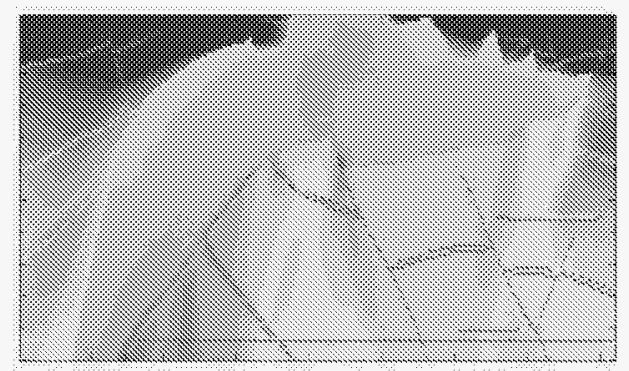

Total acquisition dip-response

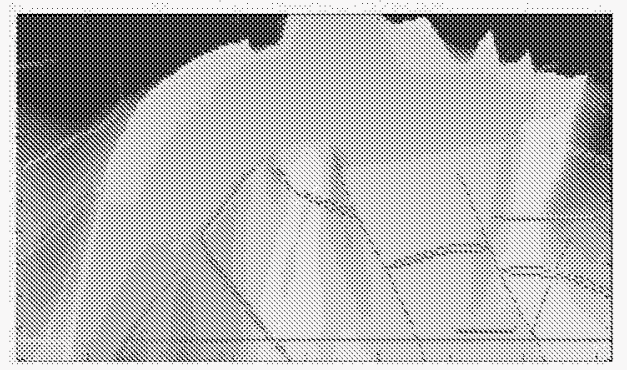

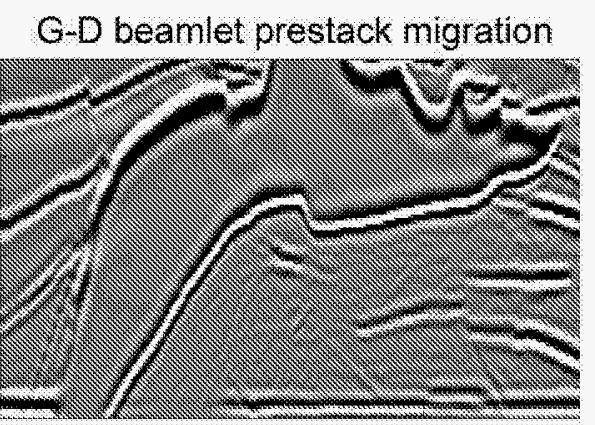

Acquisition dip-response (horizontal)

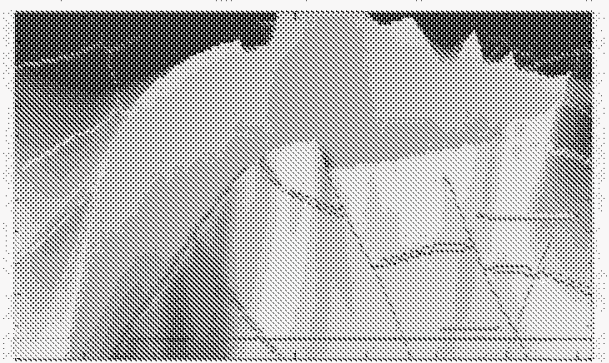

Acquisition dip-response $\left(45^{\circ} \mathrm{dip}\right)$

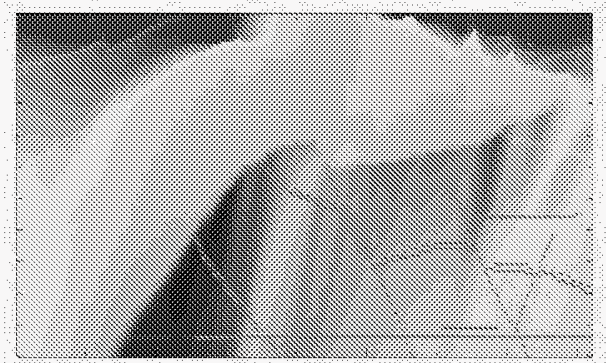

Figure 10. Left: prestack depth image (top) in comparison with the total illumination (middle) and total acquisition dip-response. Right: prestack depth image (top) in comparison with the acquisition-dipresponses for horizontal (middle) and 45 degree dip (bottom). We see good correlations between the ADRs and the reflector images of different dip angles. 

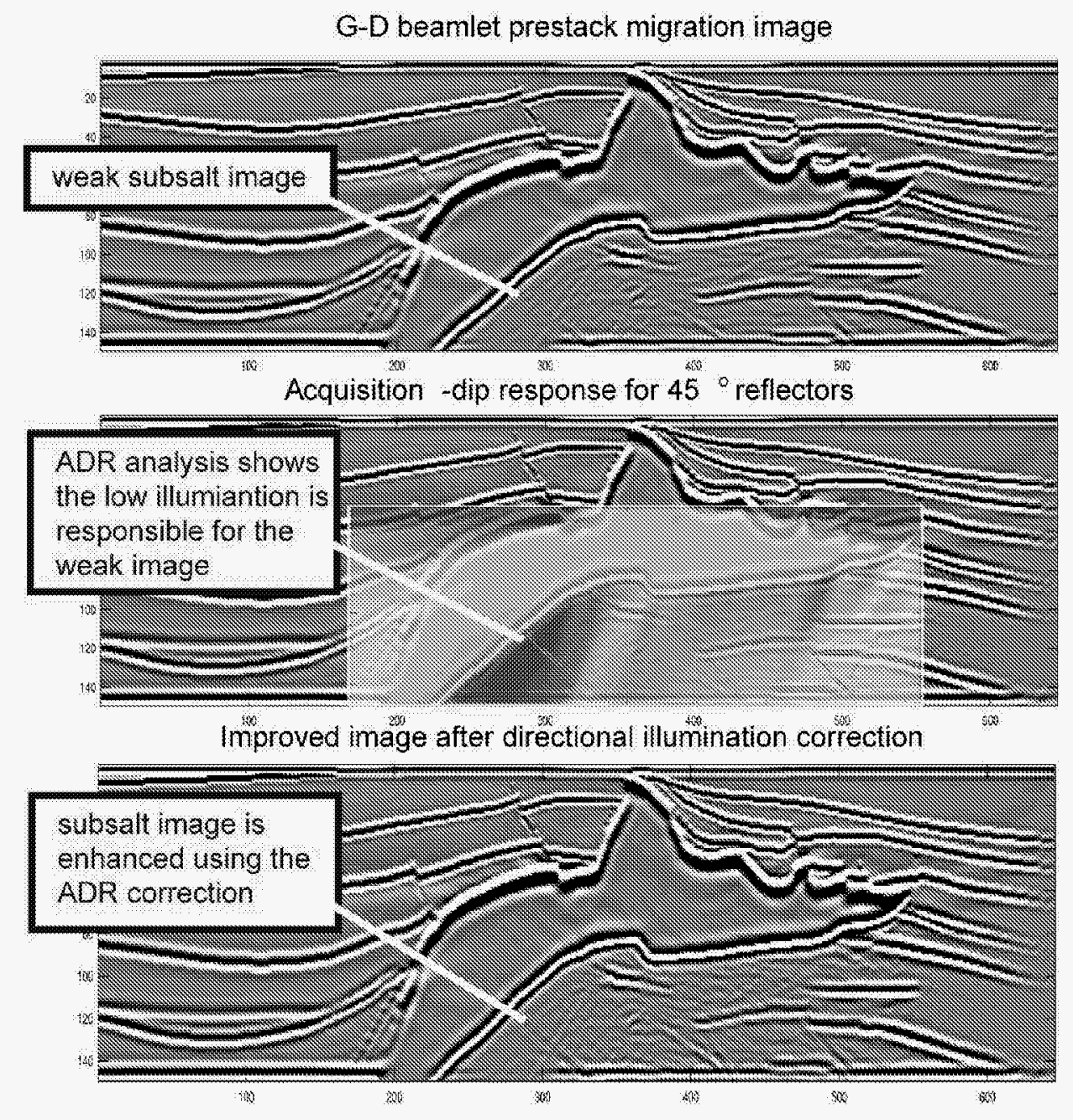

Figure 11. Amplitude correction based on illumination analysis. Top panel is the prestack depth image. In the middle, the acquisition dip response analysis is overlapped on the image. The ADR is for 45 degree dipping structure. The dark blue area indicates the poor illumination of the region from 450 , which is responsible for the weak image of the 45 degree dipping structure under the left flank of the salt body. On the bottom, the subsalt structure is enhanced using the ADR correction. 


\section{MULTICOMPONENT PRESTACK DEPTH MIGRATION USING THE ELASTIC SCREEN METHOD}

Recent developments in multicomponent seismic acquisition, such as ocean bottom cable technology, significantly increase the information in the data. From multicomponent seismic data, both P-P and P-S sections can be obtained. Because these waves carry different information about target regions, they may provide estimates of petrophysical parameters that cannot be obtained with P-wave data alone. However, more sophisticated imaging/inversion techniques are required in order to extract the new information.

Several types of methods have been proposed for elastic wave migration. Chang and McMechan $(1987,1994)$ conducted 2-D and 3-D elastic reverse-time migrations using a full wave finite-difference method. Kirchhoff elastic wave migration was implemented by Kuo and Dai (1984) and by Dai and Kuo (1986). Hokstad (2000) recently proposed a multicomponent Kirchhoff migration method and imaging conditions for multicomponent data. Zhe and Greenhalgh (1997) used potentials, instead of displacements, to propagate P- and S-waves. Some authors (e.g., Jin et al., 1998b; Sun and McMechan, 2001; Hou and Marfurt, 2002) treated P- and S-waves as scalar waves with different velocities and used a scalar wave propagator for the migration. For existing elastic wave migration approaches, the reverse-time propagator based on the full-wave finite-difference method is accurate. However, the method is usually very time consuming, especially in 3-D. The ray-based Kirchhoff method is efficient and can carry polarization information of multicomponent waves, but has difficulty dealing with many wave related phenomena. The potential approach by Zhe and Greenhalgh (1997) is very attractive. It uses a scalar potential to propagate elastic waves, and then retrieves vector waves from the potentials. However, in 3-D, the S-wave potential itself is a 3 -D vector field which reduces the advantage of this approach. Using a scalar wave propagator to simulate elastic wave propagation, although straightforward, loses polarization information carried in the multicomponent wavefield, unless otherwise specially treated (Hou and Marfurt, 2002).

The phase screen method, or split-step Fourier method (Stoffa et al., 1990), is based on the one-way wave equation. It can properly handle wave motion related phenomena. Although the original phase screen method cannot handle large scattering angles under large velocity perturbations, several modifications have been developed to improve its accuracy for wide-angles and large velocity contrasts (e.g., Ristow and Ruhl, 1994; Jin et al., 1998a; Xie and Wu, 1998; Huang et al., 1999; Huang and Fehler, 2000; Xie et al., 2000; Biondi, 2002). By processing the wavefield in both space and wavenumber domains, the screen method is also highly efficient. It provides a fast, high-quality propagator that has been successfully used for scalar wave migration.

The scalar wave screen propagator has been extended for elastic modeling of the propagation of multicomponent waves. To generalize the scalar wave screen propagator to the multicomponent elastic case, Fisk and McCartor (1991) derived the coupling term between P- and S-waves. Wu (1994, 1996) derived these couplings based on elastic scattering theory. Wild and Hudson (1998) reached a similar result using a geometrical approach. Xie and Wu $(1996,2001)$ gave equations for modeling forward propagation and reflection of multicomponent elastic waves. Similar to a scalar screen propagator, the 
elastic screen propagator neglects reverberations between screens, but can handle forward multiple-scattering effects, e.g., focusing/defocusing, diffraction, interference, and conversions between different wave types. As in the scalar wave case, the original elastic screen method is derived from a small angle approximation. Xie and $\mathrm{Wu}$ (1999) introduced a wide-angle correction for the elastic screen method. Their result improved the accuracy of elastic screen method for wide scattering angles and large velocity contrasts. Wu and Xie (1994) tested the elastic screen method as a backpropagator for multi-component elastic migration.

Based on the successful application of scalar wave screen migration and the development of elastic wave screen propagator in seismic wave modeling, we developed a multicomponent migration method based on the elastic screen propagator. The elastic screen propagator retains the advantages of the original scalar screen method and provides an efficient approach for multicomponent migration. In this chapter, we first present the formulation of the elastic screen propagator for wavefield extrapolation. Wide-angle corrections for handling wide scattering angles under large velocity contrasts are introduced. Then, vector image conditions for multicomponent P-P and P-S wave imaging are proposed. To show the feasibility of the new migration method, several 2-D synthetic data sets are generated using a full wave finite-difference method and multicomponent migrations are conducted using these synthetic data sets.

\subsection{Screen Propagator for Multicomponent Elastic Waves}

Xie and Wu (2001) gave derivations of the complex screen method for elastic wave forward propagation and reflection. Several versions of the propagators were given based on different approximations. To give equations for multicomponent elastic migration, we will follow the derivation in Xie and $\mathrm{Wu}$ (2001). Only the relevant formulation is given here, without detailed derivations. To formulate the problem in a three-dimensional heterogeneous structure, the model is divided into a sequence of horizontally layered heterogeneous thin slabs. The geometry of the model is shown in Figure 1. For simplicity, only one thin slab is marked in the figure. For analyzing wave propagation based on the one-way wave equation, the vertical direction is chosen as the main propagation direction. We use $\mathbf{x}=\mathbf{x}_{T}+z \hat{\mathbf{e}}_{z}$ as a position vector, where $\mathbf{x}_{T}=x \hat{\mathbf{e}}_{x}+y \hat{\mathbf{e}}_{y}$ is a position vector in the horizontal plane, subscript $T$ denotes the horizontal or "transverse" direction perpendicular to the main propagation direction, and $z$ is the vertical coordinate with positive direction downward. Consider forward propagated $\mathrm{P}$ - and $\mathrm{S}$-waves entering the slab from the horizontal plane at $z_{0}$. After the incident waves pass through the thin slab between $z_{0}$ and $z_{1}$, and interacting with the heterogeneities within it, there will be both incident waves and different types of forward scattered waves at the exit plane at $z_{1}$ (see Figure 1). In the frequency domain, the wavefield at $z_{1}$ can be expressed as a superposition of plane $\mathrm{P}$ - and $\mathrm{S}$-waves

$$
\mathbf{u}\left(\mathbf{x}_{T}, z_{1}\right)=\frac{1}{4 \pi^{2}} \int d \mathbf{K}_{T}\left[\mathbf{u}^{P}\left(\mathbf{K}_{T}, z_{1}\right)+\mathbf{u}^{S}\left(\mathbf{K}_{T}, z_{1}\right)\right] e^{i \mathbf{K}_{T} \cdot \mathbf{x}_{T}},
$$

where $\mathbf{u}$ is the displacement field, superscripts $P$ and $S$ denote $\mathrm{P}$ - and $\mathrm{S}$-waves, and $\mathbf{K}_{T}$ is the horizontal wavenumber of plane waves. For simplicity, the apparent frequency dependency has been omitted from equation (1) and following equations. If the slab is 
thin enough, the local Born approximation can be applied. By further invoking the smallangle approximation, the heterogeneous slab can be approximated by a homogeneous slab with background elastic parameters (P- and S-wave speeds, densities, etc.), plus a 2$\mathrm{D}$ heterogeneous "screen" in the horizontal direction. The screen concentrates all perturbations of elastic parameters into a sheet, i.e., 2-D heterogeneities without vertical structure. The interaction between the incoming wave and a heterogeneous slab can be replaced with two separated steps: the propagation in a homogeneous slab and the interaction with a 2-D heterogeneous screen. Later, we will demonstrate, such a treatment provides an efficient way to propagate elastic waves in heterogeneous models. Based on the above treatment, plane P- and S-waves $\mathbf{u}^{P}\left(\mathbf{K}_{T}, z_{1}\right)$ and $\mathbf{u}^{S}\left(\mathbf{K}_{T}, z_{1}\right)$ at the exit plane at $z_{1}$ can be expressed as (see Xie and $\mathrm{Wu}, 2001)$

$$
\begin{aligned}
& \mathbf{u}^{P}\left(\mathbf{K}_{T}, z_{1}\right)=e^{i \gamma_{\alpha} \Delta z}\left[\mathbf{u}_{0}^{P}\left(\mathbf{K}_{T}, z_{0}\right)+\mathbf{U}^{P P}\left(\mathbf{K}_{T}, z_{0}\right)+\mathbf{U}^{S P}\left(\mathbf{K}_{T}, z_{0}\right)\right], \\
& \mathbf{u}^{S}\left(\mathbf{K}_{T}, z_{1}\right)=e^{i \gamma_{\beta} \Delta z}\left[\mathbf{u}_{0}^{S}\left(\mathbf{K}_{T}, z_{0}\right)+\mathbf{U}^{S S}\left(\mathbf{K}_{T}, z_{0}\right)+\mathbf{U}^{P S}\left(\mathbf{K}_{T}, z_{0}\right)\right],
\end{aligned}
$$

where $\Delta z=z_{1}-z_{0}$ is the thickness of the slab, $\gamma_{\alpha}=\left(k_{\alpha}^{2}-K_{T}^{2}\right)^{1 / 2}$ and $\gamma_{\beta}=\left(k_{\beta}^{2}-K_{T}^{2}\right)^{1 / 2}$ are vertical wavenumbers for P- and $\mathrm{S}$-waves in the background media, $k_{\alpha}=\omega / \alpha_{0}$ and $k_{\beta}=\omega / \beta_{0}$ are $\mathrm{P}$ - and S-wavenumbers in the background medium, and $\alpha_{0}$ and $\beta_{0}$ are background $\mathrm{P}$ - and $\mathrm{S}$-wave velocities, respectively. In the above equations, $\mathbf{u}_{0}$ is the incident wavefield at the entrance plane at $z=z_{0}$. Their superscripts $P$ and $S$ denote P- and $\mathrm{S}$-waves. $\mathbf{U}$ denotes scattered waves. These scattered fields are generated by the interaction between incident waves and heterogeneities in the slab. $\mathbf{U}^{P P}$ and $\mathbf{U}^{P S}$ are scattered $\mathrm{P}$ - and $\mathrm{S}$-waves generated by the incident $\mathrm{P}$-wave, and $\mathbf{U}^{S P}$ and $\mathbf{U}^{S S}$ are scattered $\mathrm{P}$ - and $\mathrm{S}$-waves generated by the incident $\mathrm{S}$-wave. Phase shift factors $\exp \left(i \gamma_{\alpha} \Delta z\right)$ and $\exp \left(i \gamma_{\beta} \Delta z\right)$ propagate wavefields through the homogeneous background. Equations (2) and (3) indicate that, at the exit plane, the new $\mathrm{P}$-wave is composed of incident $\mathrm{P}$-wave and scattered $\mathrm{P}$-waves from incident $\mathrm{P}$ - and $\mathrm{S}$-waves, and the new $\mathrm{S}$-wave is composed of incident $\mathrm{S}$-wave and scattered $\mathrm{S}$-waves from incident $\mathrm{P}$ - and $\mathrm{S}$-waves. The process is sketched in Figure 1. For an isotropic medium, the scattered fields in equations (2) and (3) can be expressed as (Xie and $\mathrm{Wu}, 2001$ )

$$
\begin{gathered}
\mathbf{U}^{P P}\left(\mathbf{K}_{T}, z_{0}\right)=-i k_{\alpha} \Delta z \hat{k}_{\alpha} \eta^{P P} \iint d \mathbf{x}_{T}^{\prime} e^{-i \mathbf{K}_{T} \cdot \mathbf{x}_{T}^{\prime}} u_{0}^{P}\left(\mathbf{x}_{T}^{\prime}, z_{0}\right) \frac{\delta \alpha\left(\mathbf{x}_{T}^{\prime}\right)}{\alpha_{0}}, \\
\mathbf{U}^{P S}\left(\mathbf{K}_{T}, z_{0}\right)=-i k_{\beta} \Delta z \eta^{P S} \hat{k}_{\beta} \times\left\{\hat{k}_{\beta} \times \iint d \mathbf{x}_{T}^{\prime} e^{-i \mathbf{K}_{T} \cdot \mathbf{x}_{T}^{\prime}} \mathbf{u}_{0}^{P}\left(\mathbf{x}_{T}^{\prime}, z_{0}\right)\left[\left(\frac{\beta_{0}}{\alpha_{0}}-\frac{1}{2}\right) \frac{\delta \rho\left(\mathbf{x}_{T}^{\prime}\right)}{\rho_{0}}+2\left(\frac{\beta_{0}}{\alpha_{0}}\right) \frac{\delta \beta\left(\mathbf{x}_{T}^{\prime}\right)}{\beta_{0}}\right]\right\}, \\
\mathbf{U}^{S P}\left(\mathbf{K}_{T}, z_{0}\right)=-i k_{\alpha} \Delta z \eta^{S P} \hat{k}_{\alpha}\left\{\hat{k}_{\alpha} \cdot \iint d \mathbf{x}_{T}^{\prime} e^{-i \mathbf{K}_{T} \cdot \mathbf{x}_{T}^{\prime}} \mathbf{u}_{0}^{S}\left(\mathbf{x}_{T}^{\prime}, z_{0}\right)\left[\left(\frac{\beta_{0}}{\alpha_{0}}-\frac{1}{2}\right) \frac{\delta \rho\left(\mathbf{x}_{T}^{\prime}\right)}{\rho_{0}}+2\left(\frac{\beta_{0}}{\alpha_{0}}\right) \frac{\delta \beta\left(\mathbf{x}_{T}^{\prime}\right)}{\beta_{0}}\right]\right\},
\end{gathered}
$$

and

$$
\mathbf{U}^{S S}\left(\mathbf{K}_{T}, z_{0}\right)=-i k_{\beta} \Delta z \eta^{S S} \hat{k}_{\beta} \times\left\{\hat{k}_{\beta} \times \iint d \mathbf{x}_{T}^{\prime} e^{-i \mathbf{K}_{T} \cdot \mathbf{x}_{T}^{\prime}} \mathbf{u}_{0}^{S}\left(\mathbf{x}_{T}^{\prime}, z_{0}\right) \frac{\delta \beta\left(\mathbf{x}_{T}^{\prime}\right)}{\beta_{0}}\right\},
$$


where $\hat{k}_{\alpha}$ and $\hat{k}_{\beta}$ are unit wavenumber vectors for scattered $\mathrm{P}$ - and $\mathrm{S}$-waves, $\delta \alpha, \delta \beta$ and $\delta \rho$ are perturbations of $\mathrm{P}$ - and $\mathrm{S}$-wave velocities and density (they are all 2-D functions obtained from 3-D perturbations by taking an integral along the $z$-direction between $z_{0}$ and $\left.z_{1}\right), \rho_{0}$ is the background density, $\eta^{P P}, \eta^{P S}, \eta^{S P}$ and $\eta^{S S}$ are modulation factors with

$$
\begin{aligned}
\eta^{P P} & =1, \\
\eta^{P S} & =\operatorname{sinc}\left[\left(k_{\beta}-k_{\alpha}\right) \Delta z / 2\right] e^{-i\left(k_{\beta}-k_{\alpha}\right) \Delta z / 2}, \\
\eta^{S P} & =\eta^{* P S}, \\
\eta^{S S} & =1,
\end{aligned}
$$

where $\eta^{*}$ is the complex conjugate of $\eta$, and $\operatorname{sinc}(\mathrm{x})=\sin (x) / x$. Scatterings of the same wave type, i.e., P-P and S-S scatterings, modify $\mathrm{P}$ - and $\mathrm{S}$-wave phases. They resemble the scalar wave phase screen, and depend only on P- or S-wave velocity. Scattering between different wave types, i.e., P-S and S-P scattering, involve coupling between P- and Swaves. Coupling terms depend on P-wave velocity, S-wave velocity and density.

Equations (1) to (7) form the basis of the elastic screen propagator. In equations (4) to (7), the incident waves interact with heterogeneities. These operations are conducted in the horizontal spatial domain and are very efficient. Then, the spatial domain results are transformed to the wavenumber domain using fast Fourier transforms. The wavenumberdomain scattered waves given by equations (4) to (7) are substituted into equations (2) and (3). Phase shift factors in these equations propagate incident and scattered waves passing through the homogeneous background in the wavenumber-domain. These wavenumber-domain operations are, again, highly efficient. Finally, plane P- and Swaves in the wavenumber-domain are substituted into equation (1) to generate the spatial domain wavefield using an inverse fast Fourier transform. If turning waves are not of concern, the evanescent wave can be omitted because it does not propagate in the vertical direction. The integral in equation (1) only need be conducted for $\mathbf{K}_{\mathrm{T}} \mathrm{s}$ that generate real vertical wavenumbers $\gamma_{\alpha}$ and $\gamma_{\beta}$. The above description is focused on a single thin slab.

By treating the output from one thin slab as the input of the next slab and iteratively using equations (1) to (7), elastic waves can be propagated through the entire model.

In summary, the model parameters are separated into two parts, the background parameters and the perturbations. The wave propagation through a thin slab is decomposed into a series of steps. The interactions between the incoming waves and the perturbations are conducted in the spatial domain. Plane wave propagation through the background medium is conducted in the wavenumber domain. In both domains, the calculations are local and highly efficient. There is no time-consuming spatial or wavenumber domain convolution involved. The forward and inverse fast Fourier transforms alternate the wavefield between the two domains.

Another advantage of the elastic screen propagator is that wavefield coupling and propagation are treated separately. As mentioned above, the $\mathbf{U}^{P S}$ and $\mathbf{U}^{S P}$ in equations (2) and (3) represent couplings between $\mathrm{P}$ - and $\mathrm{S}$-waves. This gives us the flexibility to turn "on" or "off" certain couplings in the spatial-domain. For regions where P- and S-wave velocities change smoothly, we can neglect the coupling between $\mathrm{P}$ - and $\mathrm{S}$-waves in equations (5) and (6). If couplings in the entire slab can be omitted, $\mathbf{U}^{P S}$ and $\mathbf{U}^{S P}$ in equations (2) and (3) can be neglected. Using the Rytov approximation, equations (2) and (3) can be rewritten as (Xie and $\mathrm{Wu}, 2001)$ 


$$
\begin{gathered}
\mathbf{u}^{P}\left(\mathbf{K}_{T}, z_{1}\right)=e^{i \gamma_{\alpha} \Delta z} \iint d \mathbf{x}_{T}^{\prime} e^{-i \mathbf{K}_{T} \cdot \mathbf{x}_{T}^{\prime}} \mathbf{u}_{0}^{P}\left(\mathbf{x}_{T}^{\prime}, z_{0}\right) e^{-i k_{\alpha} \Delta z \delta \alpha / \alpha_{0}}, \\
\mathbf{u}^{S}\left(\mathbf{K}_{T}, z_{1}\right)=-e^{i \gamma_{\beta} \Delta z} \hat{k}_{\beta} \times\left[\hat{k}_{\beta} \times \iint d \mathbf{x}_{T}^{\prime} e^{-i \mathbf{K}_{T} \cdot \mathbf{x}_{T}^{\prime}} \mathbf{u}_{0}^{S}\left(\mathbf{x}_{T}^{\prime}, z_{0}\right) e^{-i k_{\beta} \Delta z \delta \beta / \beta_{0}}\right] .
\end{gathered}
$$

Equations (12) and (13), together with equation (1), form single wave type propagators for $\mathrm{P}$ - and $\mathrm{S}$-waves. When processing the wavefield extrapolation, based on the model complexity and the migration requirement, we can easily switch to different propagators in different parts of the model. For example, we can use equations (2) and (3) in complex regions with fast velocity changes; use equations (12) and (13) in thick layers with smoothly changing velocities; or turn off wave couplings in salt bodies with nearly constant velocities. If we totally omit coupling between $\mathrm{P}$ - and S-waves along the entire propagation path (other than the target reflector), we can use equations (12) and (13) in the entire model. The result will be similar to a converted wave migration except here the process is for multicomponent waves and the polarization information is preserved.

To do the migration, we have to propagate downgoing waves from the source to the target and backpropagate reflected waves from the receivers to the target. The propagators derived in this section can be used for forward propagation. The complex conjugates of these propagators can be used for backpropagation. Alternatively, one can use the forward propagator together with the time-reversed data (or equivalently, the complex conjugate of the frequency domain data) to conduct a back propagation. The later case is equivalent to a reverse-time propagator in the frequency domain, except it is a one-way propagator. The advantage of the latter is that one propagator serves for both up- and down-going waves (when both of them are in the same wave type). This saves some effort in managing calculations.

\subsection{Wide-Angle Correction for Elastic Propagators}

The elastic screen propagators obtained in the previous section are derived from the perturbation theory and a small-angle approximation. They cannot handle wide scattering angles under large velocity contrasts. However, real situations often contain large velocity contrasts. For scalar waves, several approaches have been employed to improve the accuracy of the screen propagator for large velocity contrasts and wide scattering angles (e.g., Ristow and Ruhl, 1994; Jin et al., 1998a; Xie and Wu, 1998; Huang and Fehler, 2000; Biondi, 2002). A similar method can be adopted for elastic waves (Xie and $\mathrm{Wu}, 1999)$. As mentioned before, the coupling and propagation are calculated separately in screen methods. Having obtained the coupling terms, the P- and S-waves can propagate through the background slab independently; i.e., their propagations are decoupled. This permits us to modify the P- and S-waves separately. The wide-angle correction used for scalar waves can be adopted for elastic waves. To apply the wideangle correction, screen solutions $\mathbf{u}^{P}$ and $\mathbf{u}^{S}$ are first obtained from the coupled equations (2) and (3) or from the single wave type equations (12) and (13). Corrections are then performed on $\mathbf{u}^{P}$ and $\mathbf{u}^{S}$, respectively. Finally, the corrected results replace $\mathbf{u}^{P}$ and $\mathbf{u}^{S}$ in equation (1) to give the modified solution. As for scalar waves, the correction is accomplished by solving an implicit finite-difference problem in the spatial domain (e.g., Ristow and Ruhl, 1994; Jin et al., 1998; Xie and Wu, 1998, 1999; Huang and Fehler, 2000; Biondi, 2002), which usually gives better stability than the wavenumber domain 
method. These processes can fit easily into a dual domain operation for wavefield extrapolation.

\subsection{Imaging Conditions}

An imaging process is aimed at recovery of interface properties from incident and scattered waves. These properties should not be affected by the configuration of the observation system, nor depend on the coordinate system chosen to conduct the calculation. In this study, both up- and down-going waves are extrapolated using multicomponent screen propagators, and polarizations are preserved throughout the propagation. We developed a vector imaging condition for the multicomponent wave imaging. The imaging condition is based on the principle that at the reflection point, the up- and down-going waves are in phase (coincidence of incident and reflected waves). This is the same as for scalar waves except we have two back-propagated upgoing waves, i.e., P-P and P-S waves. Following Mittet et al. (1995), the multicomponent imaging conditions for P-P and P-S converted waves can be written as

$$
\left.I^{P P}\left(\mathbf{x}_{T}, z\right)=\int C^{P-P P}\left[\hat{\mathbf{e}}^{P} \cdot \mathbf{u}^{P}\left(\mathbf{x}_{T}, z, \omega\right)\right]\left[\hat{\mathbf{e}}^{P P} \cdot \mathbf{u}^{P P}\left(\mathbf{x}_{T}, z, \omega\right)\right]\right]^{*} d \omega,
$$

and

$$
I^{P S}\left(\mathbf{x}_{T}, z\right)=\int C^{P-P S}\left[\hat{\mathbf{e}}^{P} \cdot \mathbf{u}^{P}\left(\mathbf{x}_{T}, z, \omega\right)\right]\left[\hat{\mathbf{e}}^{P S} \cdot \mathbf{u}^{P S}\left(\mathbf{x}_{T}, z, \omega\right)\right]^{*} d \omega,
$$

where $\mathbf{u}^{P}$ is the downgoing P-wave from the source, $\mathbf{u}^{P P}$ and $\mathbf{u}^{P S}$ are upgoing P-P and P-S waves back-propagated from the receivers, $\hat{\mathbf{e}}^{P}, \hat{\mathbf{e}}^{P P}$ and $\hat{\mathbf{e}}^{P S}$ are the corresponding unit polarization vectors, "*” denotes complex conjugate, and $C^{P-P P}$ and $C^{P-P S}$ are normalization factors which can be chosen from the various versions discussed by Mittet et al. (1995).

Obtaining polarization vectors $\hat{\mathbf{e}}^{P}, \hat{\mathbf{e}}^{P P}$ and $\hat{\mathbf{e}}^{P S}$ is not straightforward. In the frequency domain, the wavefields $\mathbf{u}^{P}, \mathbf{u}^{P P}$ and $\mathbf{u}^{P S}$ are vectors of complex numbers. We can write $\mathbf{u}^{P}=u^{P} \hat{\mathbf{e}}^{P}, \mathbf{u}^{P P}=u^{P P} \hat{\mathbf{e}}^{P P}$ and $\mathbf{u}^{P S}=u^{P S} \hat{\mathbf{e}}^{P S}$, where $u^{P}, u^{P P}$ and $u^{P S}$ are complex amplitudes. The difficulty is, unlike real valued vectors, their exact polarization directions cannot be uniquely determined from their components. There is an ambiguity of $\exp (i \pi)$ which can appear in either the amplitudes or the directions of these wavefields. This further introduces ambiguity in the polarization of the image. For easier discussion, we assume that at the imaging point both source and receiver waves are linearly polarized. From scattering theory (e.g., Wu and Aki, 1985), the incident and scattered waves form an in-plane problem. The plane is perpendicular to the vector $\hat{\mathbf{e}}^{P} \times \hat{\mathbf{e}}^{P P}$ or $\hat{\mathbf{e}}^{P} \times \hat{\mathbf{e}}^{P S}$ and contains the scattering point. Figure 2 shows the P-P and P-S reflection geometry within the plane. Although polarization vectors cannot be uniquely determined, a pair of consistent polarization directions for incident and reflected waves can be obtained. Figure 2 shows polarization pairs for P-P and P-S reflections for a "positive" impedance interface (Aki and Richards, 1980, p. 148). Once polarization pairs are determined, complex wavefields $\mathbf{u}^{P}, \mathbf{u}^{P P}$ and $\mathbf{u}^{P S}$ can be projected onto these directions. Then, correct complex magnitudes can be obtained and applied in the imaging. Taking P-S wave image as an example, the process can be summarized as: (a) Find two possible polarization directions for the downgoing P-wave. (b) Choose the direction towards the scattering point as $\hat{\mathbf{e}}^{P}$. (c) Find two possible polarization directions for the reflected $S$-wave. (d) 
According to the convention shown in Figure 2, choose the correct $\hat{\mathbf{e}}^{P S}$ from the two directions. (e) Replace $\hat{\mathbf{e}}^{P}$ and $\hat{\mathbf{e}}^{P S}$ into equation (15) to calculate the P-S image. The imaging condition uses wavefield information at the imaging point. It does not rely on the locations of the source and receivers nor any specific coordinate system. In a complicated wavefield, polarizations are not always perfectly linear. This may cause noise in the image. Summing up contributions from multiple frequencies and sources can reduce these errors.

\subsection{Numerical Examples}

\subsubsection{Synthetic Data Sets}

The multicomponent propagator and imaging conditions are tested here with 2-D numerical examples. All synthetic data sets used in this paper are generated using a fullwave fourth-order elastic finite-difference algorithm (Xie and Lay, 1994). A P-wave explosion source is used in all calculations. To simplify the treatment, absorbing boundaries are used for all sides of the model; no free-surface condition is applied. Before conducting migrations, $\mathrm{P}$ - and $\mathrm{S}$-waves are separated by calculating the divergence and curl of the coupled wavefield. The treatment is similar to those used by Zhe and Greenhalgh (1997), and Sun et al. (2001a, b). In our calculation, the separation is performed in the finite-difference code. Direct arrivals have been eliminated from these synthetic data. Figure 3 contains a typical shot record. The separation of P- and S-waves causes some waveform distortions. Zhe and Greenhalgh (1997), and Sun et al. (2001a, b) suggested algorithms for correcting these distortions.

\subsubsection{Migration/Imaging}

All migrations in this section are for common source data. Single wave type propagators (12) and (13) combined with wide-angle modifications are used for both the downgoing $\mathrm{P}$-wave and the upgoing $\mathrm{P}-\mathrm{P}$ and $\mathrm{P}-\mathrm{S}$ waves. An explosive $\mathrm{P}$-wave source is used at the first screen to generate a downgoing wavefield. Equations (14) and (15) are used for imaging. In cases where multiple sources are used, partial images from different sources are stacked for obtaining the final image.

\subsubsection{Simple Model With One Reflector}

The first example is a simple two-layer velocity model (Figure 4a). A single shot is located at the center on the surface. There are 400 receivers located on the surface with offsets from $-2000 \mathrm{~m}$ to $+2000 \mathrm{~m}$. For calculating synthetic data, $d x=d z=5 \mathrm{~m}$ and $d t=$ $0.8 \mathrm{~ms}$. For migration, we use $d x=d z=10 \mathrm{~m}$ and $d t=8 \mathrm{~ms}$. Figures $4 \mathrm{~b}$ and $4 \mathrm{c}$ contain the P-P image and the P-S converted wave image, respectively. Both images reveal the interface. Several features can be seen from this simple model. Since the S-wave scattering angle is smaller than that of the P-wave, the P-S image has a wider angle coverage. With the application of the vector imaging condition, the converted wave image has the correct polarization on both sides of the source. This allows us to construct 
the image by stacking results from multiple sources. Because of the shorter wavelength of the $S$-wave, the resolution of the P-S image is slightly higher than that of the P-P image.

\subsubsection{Three-Layer Model}

The second example is a three-layer model (Figure 5a). Sixteen shots on the surface are used to illuminate the structure. Their locations are shown in Figure 5a. Each shot has 160 receivers with offsets from $-1600 \mathrm{~m}$ to $1600 \mathrm{~m}$. To calculate synthetic seismograms using the finite-difference method, we used a grid with $d x=d z=5 \mathrm{~m}$, and $d t=0.5 \mathrm{~ms}$. For the migration, a grid with $d x=d z=20 \mathrm{~m}$, and $d t=4 \mathrm{~ms}$ is used. The stacked P-P image and P-S converted wave image are shown in Figures $5 \mathrm{~b}$ and $5 \mathrm{c}$, respectively. As a consequence of the vector image condition, converted wave images from each shot can be directly stacked to generate the final P-S wave image. Both P-wave and converted wave image qualities are good. As in Figure 4, the P-S wave image shows slightly higher resolution.

\subsubsection{Two-Dimensional Elastic SEG/EAGE Salt Model}

The next model is a modified version of a 2-D slice through the SEG/EAGE salt model (O'Brien and Gray, 1996; Aminzadeh et al., 1997). The original salt model is an acoustic model with only sound wave speed. To make this model suitable for elastic modeling/migration, several modifications have been made. The sound wave speed is used as the P-wave speed. The S-wave speed is obtained from the P-wave speed by assuming Lamè constant $\lambda=\mu$ or, equivalently, $\alpha=1.73 \beta$. The density $\rho$ can be determined based on Gardner et al.'s (1974) relation (Sheriff, 1991, p. 135) or Birch's Law (see for example: Fowler, 1990, p. 78; Sheriff, 1991, p. 27). Our current elastic finite-difference code does not handle a water layer with $\mu=0$. We eliminated the top water layer in the original SEG/EAGE salt model by filling it with a layer of material with similar properties to the sub-water layer. Figure 6a shows the P-wave velocity of the modified model. A velocity grid with $d x=d z=6 \mathrm{~m}$, and $d t=1 \mathrm{~ms}$ is used to generate the synthetic data set, while a grid with $d x=d z=24 \mathrm{~m}$, and $\mathrm{dt}=8 \mathrm{~ms}$ is used for multicomponent migration. One hundred and twenty shots on the surface are used to illuminate the structure (as a comparison, the original 2-D acoustic data set is composed of 325 shots). For each shot, we used 160 receivers with offsets from $-1950 \mathrm{~m}$ to $1950 \mathrm{~m}$.

The migrated P-P and P-S images are shown in Figures $6 \mathrm{~b}$ and $6 \mathrm{c}$, respectively. The salt body is well imaged as is the flat reflector at the bottom of the model. Thus, even with the existence of a high-speed salt body, the wide-angle propagator correctly extrapolates P- and S-waves to the bottom of the model. Generally speaking, the P-P image is better than the P-S image because a reflected P-wave usually has more energy. In the sub salt region, the incident angle for the incoming $\mathrm{P}$-wave is small (nearperpendicular incidence) and the $\mathrm{P}$ to $\mathrm{S}$ reflection is weak. This causes very weak converted wave images for many of the sub salt structures. Weak coherent noise can be seen both inside and below the salt body; these coherent noise are mainly from locally converted waves in the up- and down-going legs.

\subsection{Some Discussions}


The current study focuses on the multicomponent screen propagator and the vector image conditions. The separation of $\mathrm{P}$ - and S-waves is simply performed within the finite-difference code used for generating synthetic data. For field data, the separation of $\mathrm{P}$ - and $\mathrm{S}$-waves from multicomponent records is an important issue. Both Zhe and Greenhalgh (1997) and Sun et al. (2001a, b) have suggested using a full wave elastic finite-difference reverse time propagator to extrapolate the mixed wave downward for a small distance. Then separate $\mathrm{P}$ - and $\mathrm{S}$-waves within the finite-difference code by calculating the divergence and curl of the displacement field. This technique can also solve the free-surface condition. Other techniques are also proposed to solve the P- and S-wave separation (e.g., Amundsen and Reitar, 1995; Jin et al. 1998b). Theoretically, these methods can be used for processing field data. However, their robustness still needs to be tested.

All the numerical examples tested in this paper are conducted using the single wave type propagators in 2-D models. However, the formulation presented in this research is for full 3-D migration and includes coupled waves. Although only a P-wave source is discussed in this paper, S-wave sources, or sources generating mixed P- and S-waves can be easily included. Imaging conditions for $\mathrm{S}$-wave incidence can also be derived. In our numerical examples, both sources and receivers are located at the same depth in the model, but the current method also works where sources and receivers are located at different depths. For example, sources can be near the surface while the receivers lie on the ocean bottom or are in vertical cables.

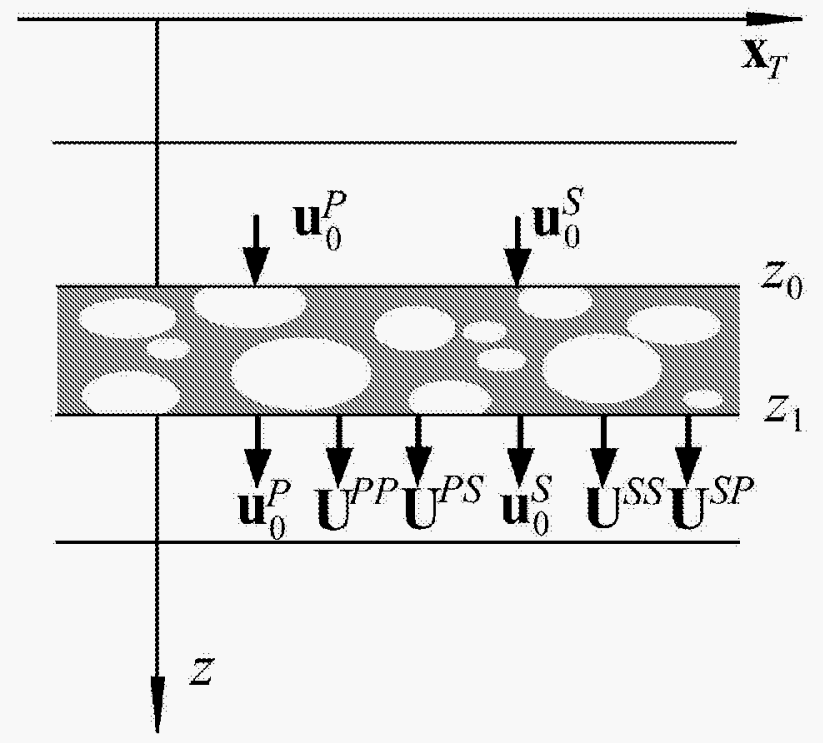

FIG. 1. A sketch showing incident $\mathrm{P}$ - and $\mathrm{S}$-waves and various types of scattered waves after they interact with a horizontal inhomogeneous thin slab. At the entrance plane $z_{0}$, there are incident $\mathrm{P}$-wave $\mathbf{u}_{0}^{P}$ and incident $\mathrm{S}$-wave $\mathbf{u}_{0}^{S}$. At the exit plane $\mathrm{z}_{1}$, after interacting with the inhomogeneous slab, there are incident waves and scattered waves. $\mathbf{U}^{P P}$ and $\mathbf{U}^{P S}$ are scattered $\mathrm{P}$ - and $\mathrm{S}$-waves generated by the incident $P$-wave, and $\mathbf{U}^{S P}$ and $\mathbf{U}^{S S}$ are scattered $\mathrm{P}$ - and $\mathrm{S}$-waves generated by the incident $\mathrm{S}$-wave. 

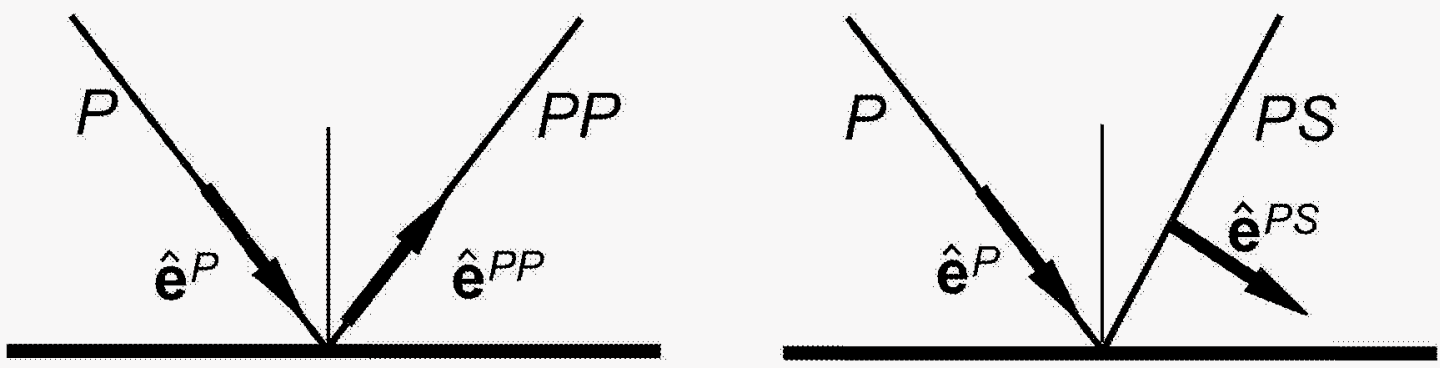

FIG. 2. Sign conventions for polarizations. $\hat{\mathbf{e}}^{P}, \hat{\mathbf{e}}^{P P}$ and $\hat{\mathbf{e}}^{P S}$ are polarization vectors for $\mathrm{P}$, $\mathrm{P}-\mathrm{P}$ and P-S waves, respectively. The reflector is not necessarily horizontal. The P-P and $\mathrm{P}-\mathrm{S}$ polarization pairs shown in this figure correspond to a positive impedance interface.
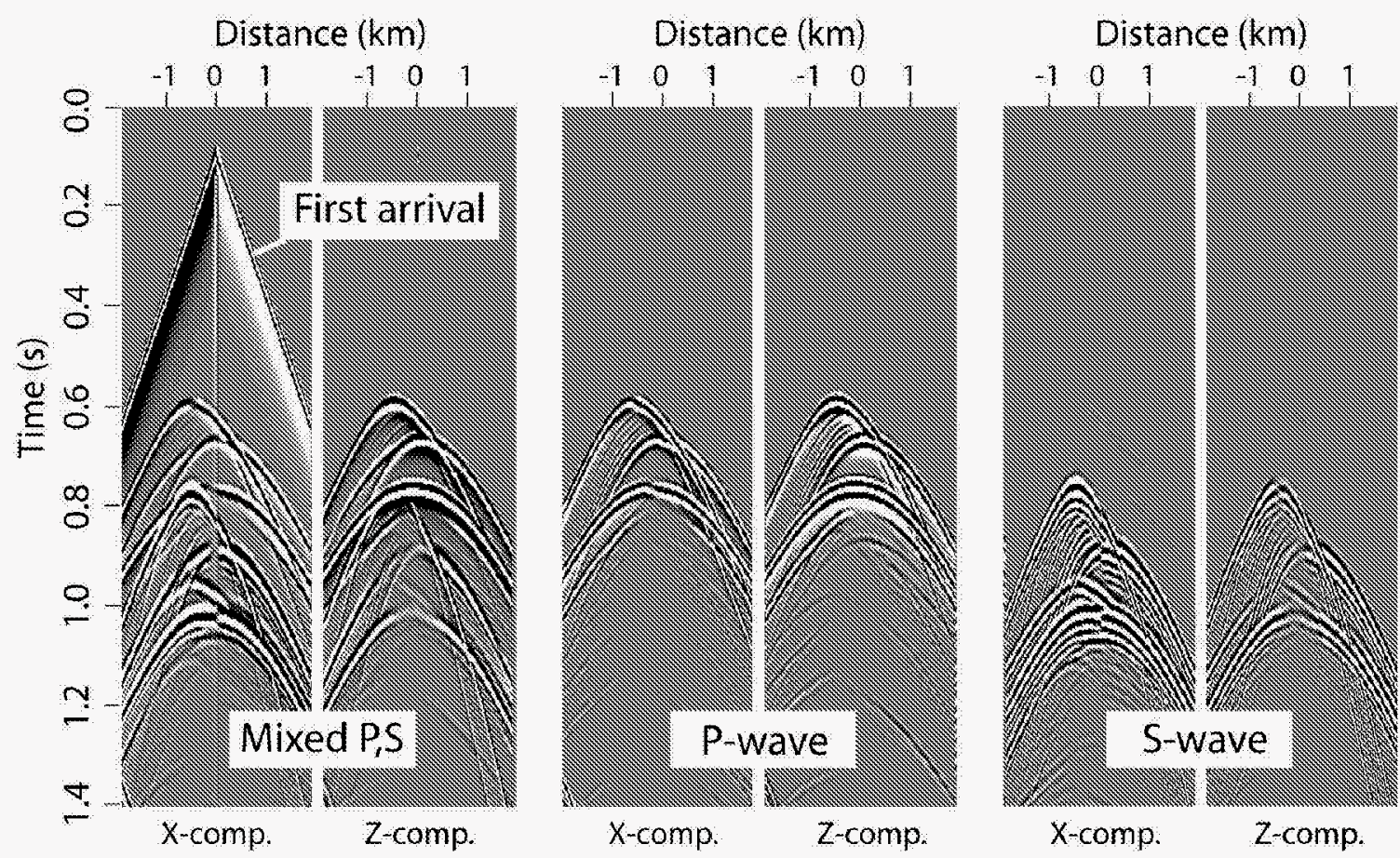

FIG. 3. A typical synthetic common-source record. In the left panel is a mixed P- and Swave record. In the right two panels are the separated pure P- and S-wave records. Within each panel, on the left is the horizontal component and on the right is the vertical component. First arrivals are eliminated from the separated P- and S-waves. 


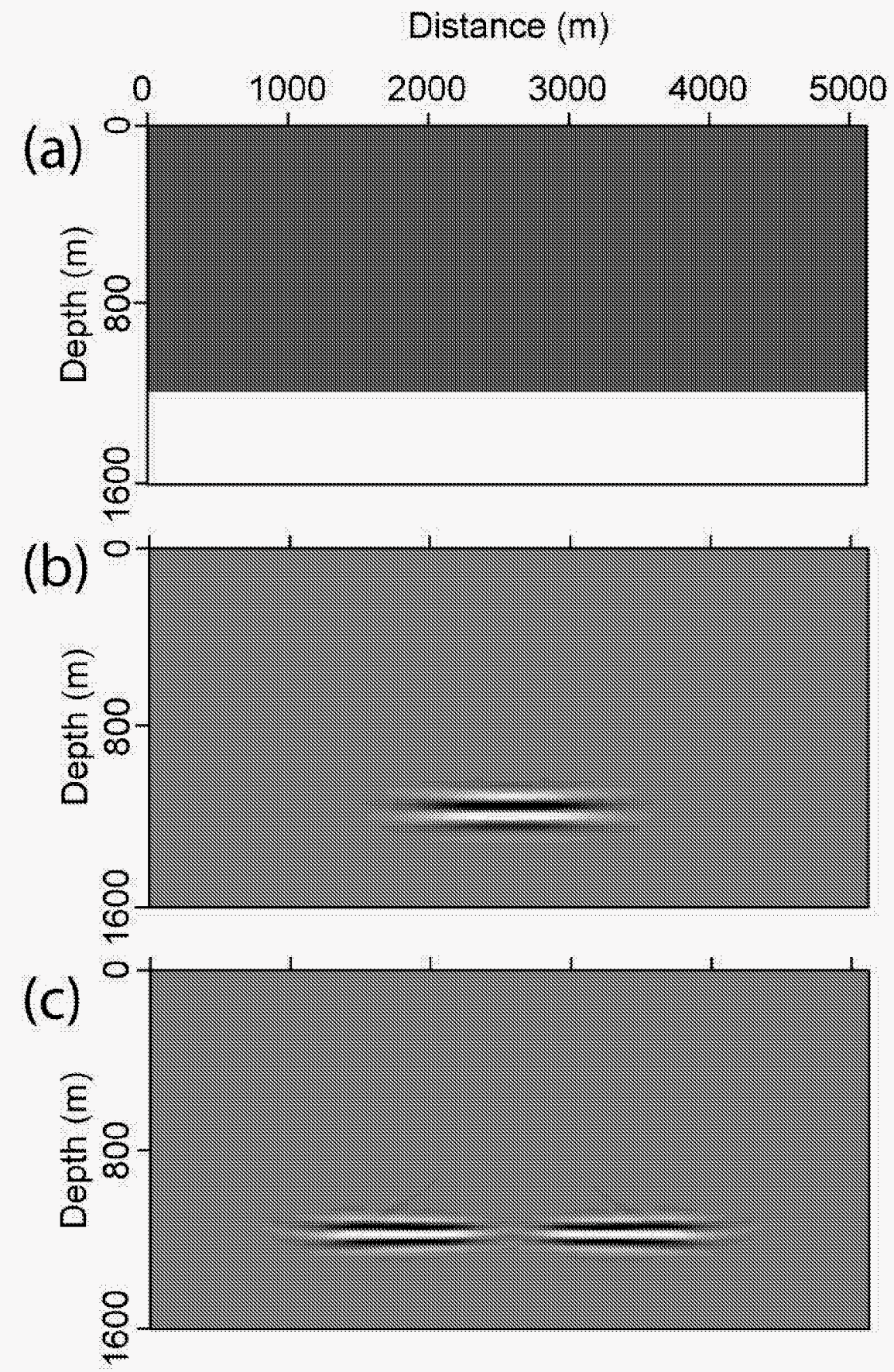

FIG. 4. Single shot prestack imaging. (a) velocity model, (b) P-P image, and (c) P-S converted wave image. The velocities and densities are $\alpha_{1}=3.5 \mathrm{~km} / \mathrm{s}, \beta_{1}=2.0 \mathrm{~km} / \mathrm{s}, \rho_{1}=2.0$ $\mathrm{g} / \mathrm{cm}^{3}$, and $\alpha_{2}=4.5 \mathrm{~km} / \mathrm{s}, \beta_{2}=2.4 \mathrm{~km} / \mathrm{s}, \rho_{2}=2.2 \mathrm{~g} / \mathrm{cm}^{3}$, respectively. 

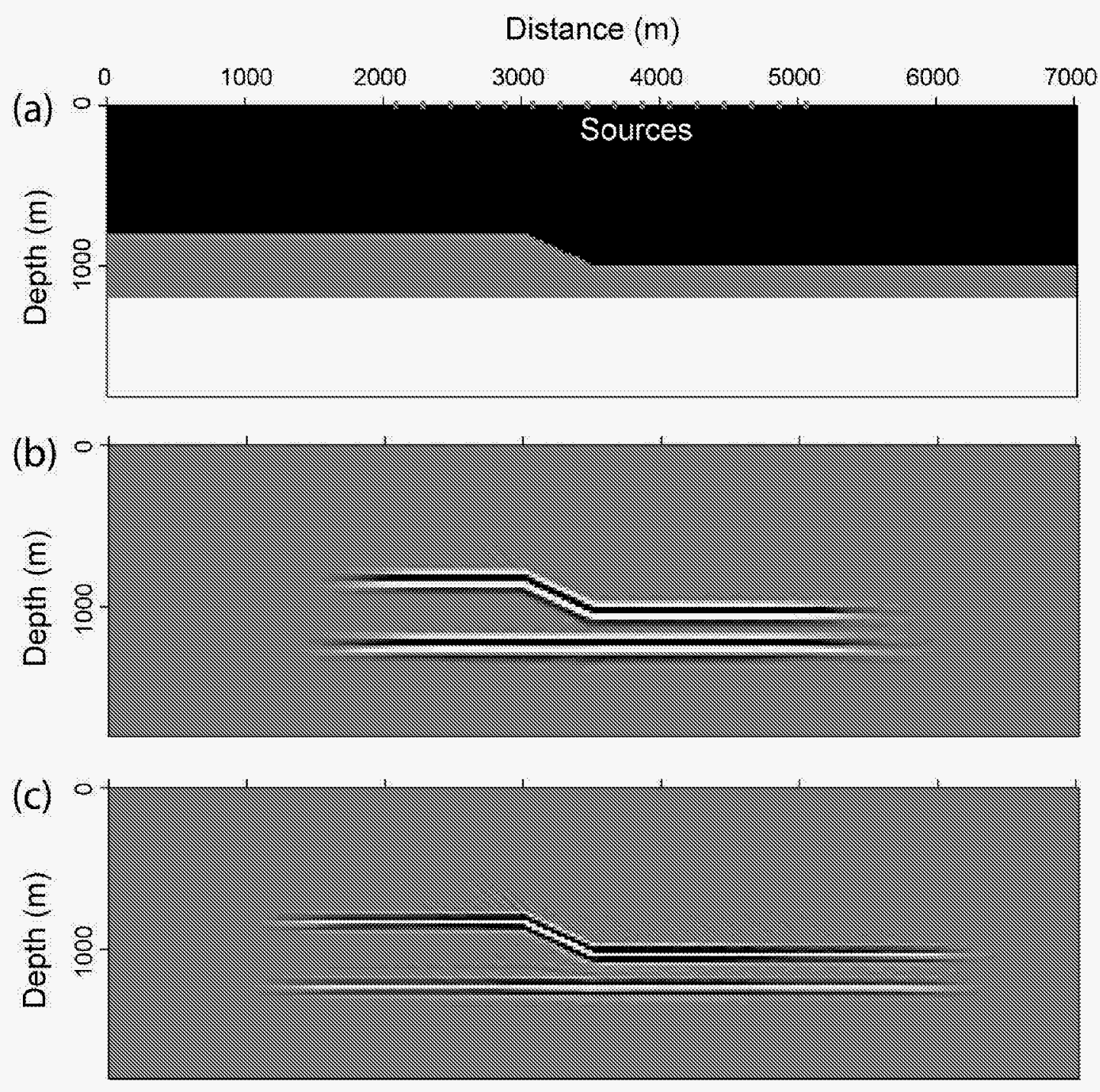

FIG 5. Migrated images of a three-layer model. (a) velocity model, (b) P-P image, and (c) $\mathrm{P}-\mathrm{S}$ converted-wave image. The velocity and density parameters are $\alpha_{1}=3.5 \mathrm{~km} / \mathrm{s}, \beta_{1}=2.0$ $\mathrm{km} / \mathrm{s}, \rho_{1}=2.0 \mathrm{~g} / \mathrm{cm}^{3}, \alpha_{2}=4.0 \mathrm{~km} / \mathrm{s}, \beta_{2}=2.4 \mathrm{~km} / \mathrm{s}, \rho_{2}=2.2 \mathrm{~g} / \mathrm{cm}^{3}$ and $\alpha_{3}=4.5 \mathrm{~km} / \mathrm{s}, \beta_{3}=2.6$ $\mathrm{km} / \mathrm{s}, \rho_{3}=2.4 \mathrm{~g} / \mathrm{cm}^{3}$, respectively. Sixteen shots are used to illuminate the model. Note that partial images from individual shots are correctly stacked to generate a P-S converted wave image. 

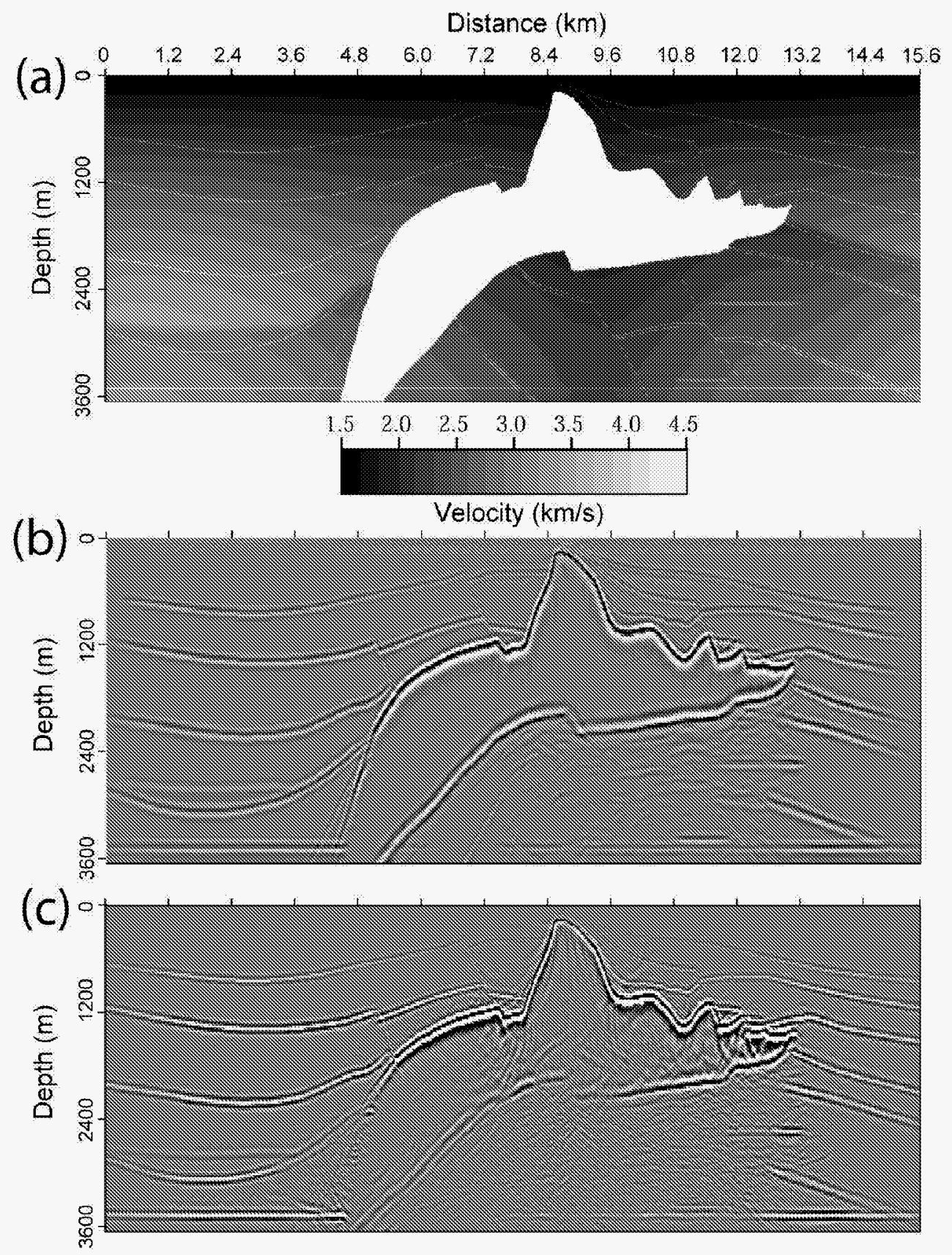

FIG. 6. Migrated images of the elastic SEG/EAGE salt model. (a) Modified velocity model (P-wave), (b) P-P image, and (c) P-S converted wave image. 


\section{CONVERTED WAVE PATH (C-PATH) IMAGING FOR STEEP SUBSALT STRUCTURES}

Converted waves have been demonstrated to be promising for imaging beneath highvelocity structures, such as in the cases of sub-basalt and sub-salt imaging. Converted waves can be used to image local conversion points (C-wave imaging for commonconversion points) (Thomsen, 1998; Kendall et al., 1998; O'Brien et al., 1999), or to improve the illumination and receiving coverages using conversion paths (Purnell,1992; Li et al., 1998; Jones and Gaiser, 1999). We will focus on the application of converted waves for the improvement of illumination coverage for subsalt imaging.

\subsection{Properties of the Converted Waves}

It is known that converted $\mathrm{S}$ waves can penetrate high-velocity salt structures with much less defocusing, and avoid the deadly critical/post-critical reflections of $\mathrm{P}$ waves which totally mask the $\mathrm{P}$ wave illumination for steep structures beneath the salt. Because of the closeness of S velocity of salt and the P velocity of sub-salt medium, P-S conversion is quite efficient at the salt boundary. Figure 1 shows the energy transmission coefficients of $\mathrm{P}$ wave (solid line) and $\mathrm{P}-\mathrm{S}$ converted wave (the $\mathrm{C}$-wave) (dotted line), respectively, for a sediment-salt interface (sediment: $\mathrm{Vp}=2.6 \mathrm{~km} / \mathrm{s}, \mathrm{Vs}=1.5 \mathrm{~km} / \mathrm{s}, \rho=2.2 \mathrm{~g} / \mathrm{cm}^{3}$; salt: $\mathrm{Vp}=4.48 \mathrm{~km} / \mathrm{s}, \mathrm{Vs}=2.59 \mathrm{~km} / \mathrm{s}, \rho=2.1 \mathrm{~g} / \mathrm{cm}^{3}$. At small incident angles, $\mathrm{P}$ transmission is favorable. However, beyond the critical angle (here is 35 degrees), no $\mathrm{P}$ energy is transmitted, while $\mathrm{C}$-waves still carry substantial energy. The peak energy transmission coefficient is about 0.3, corresponding to a peak displacement larger than half of the incident $\mathrm{P}$ displacement. We see also that $\mathrm{C}$-waves still carry enough energy even for large angle incidence up to 70 degrees. This is important for sub-salt imaging of steep structures. To see the illumination coverage and the transmission efficiency of different wave paths, we carry out some theoretical transmission calculations as illustrated schematically in Figure 2. Assuming the P to P normal reflection is the most energetic one for sub-salt faults, the illumination efficiency as a function of dip angles can be calculated through the angle dependence of $\mathrm{P}$ wave incidence for the three layer model. The high-velocy salt layer is in the middle. The energy ratios of transmitted/incident waves for different conversion paths are plotted in Figure 3. On the left of Figure 3 is the case where the wavepath inside the salt is of $\mathrm{P}$-wave; on the right, $\mathrm{S}$-wave inside salt. We see that although the received signals for small-angle incidence (nearly horizontal reflections) are dominanted by $\mathrm{P}$ waves (PPP), beyond the critical angle (of $\mathrm{P}$ wave) the converted waves still carry substantial energy to the surface. The peak signals of PSP and PSS are at different incident angles, but they are in the same level. In this work we will mainly interested in PSP and PSS which have a C-wave path inside the salt. Since we consider only the normal reflections from the sub-salt faults, the corresponding prestack migration wave paths will be either PSPPSP or PSPPSS. 


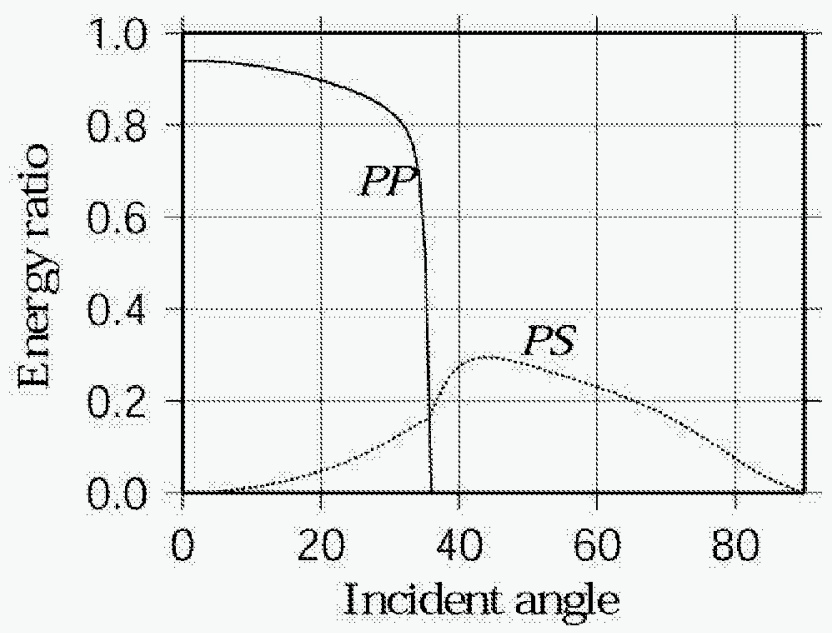

Figure 1. Energy transmission coefficients for PP and PS waves on sediment-salt nterface.

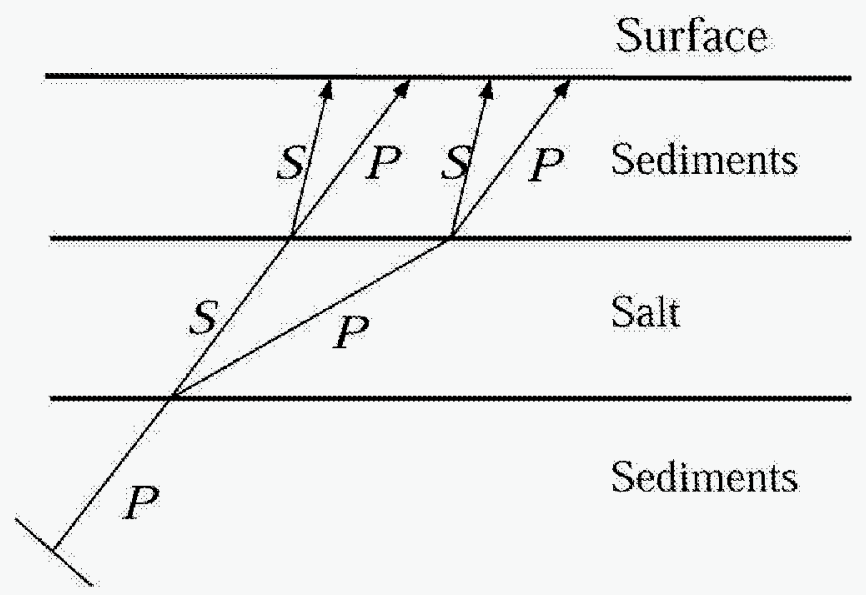

Figure 2. Schematic illustration of different wave paths for sub-salt structure illumination.
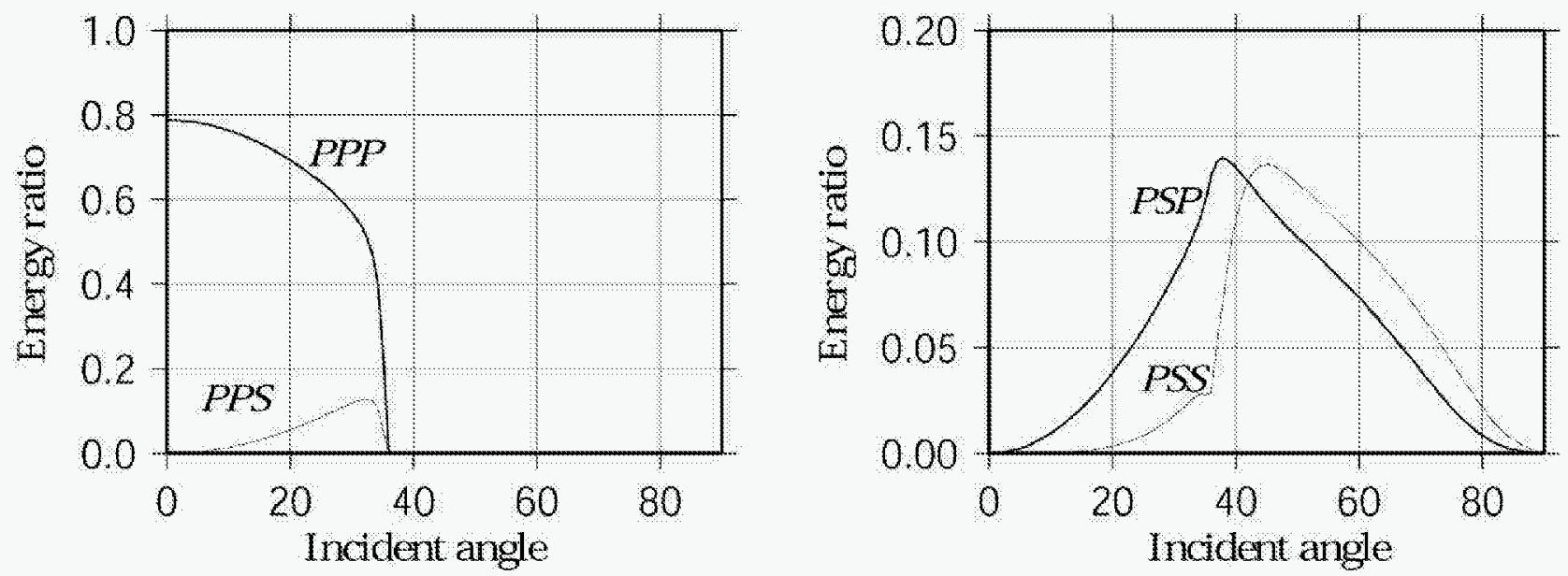

Figure 3. Energy transmission effeciency for different wave paths. The transmission coefficients are normalized to the incident P-wave energy. 

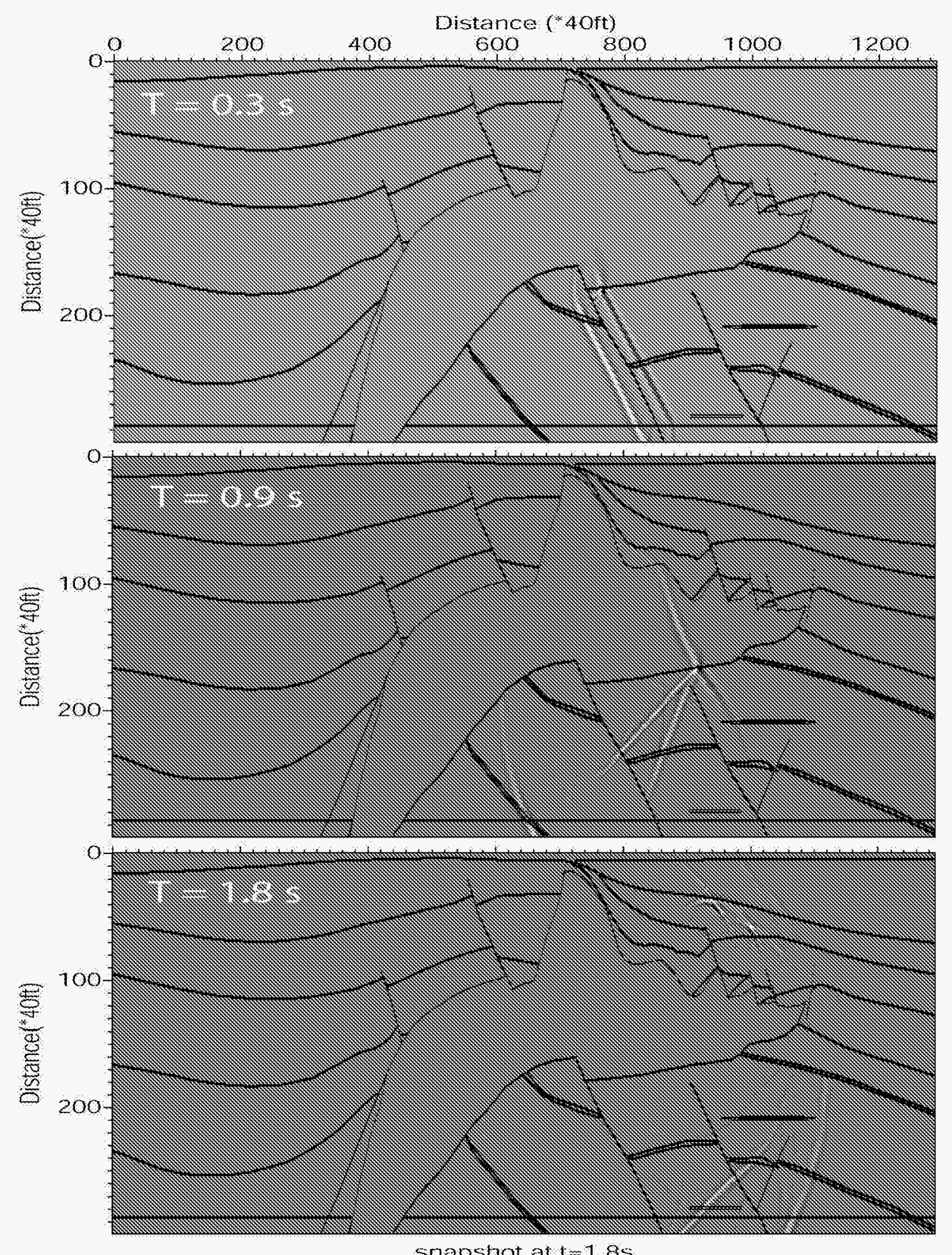

Figure 4. Snapshots when exploding reflectors are located at sub-salt fault B. 

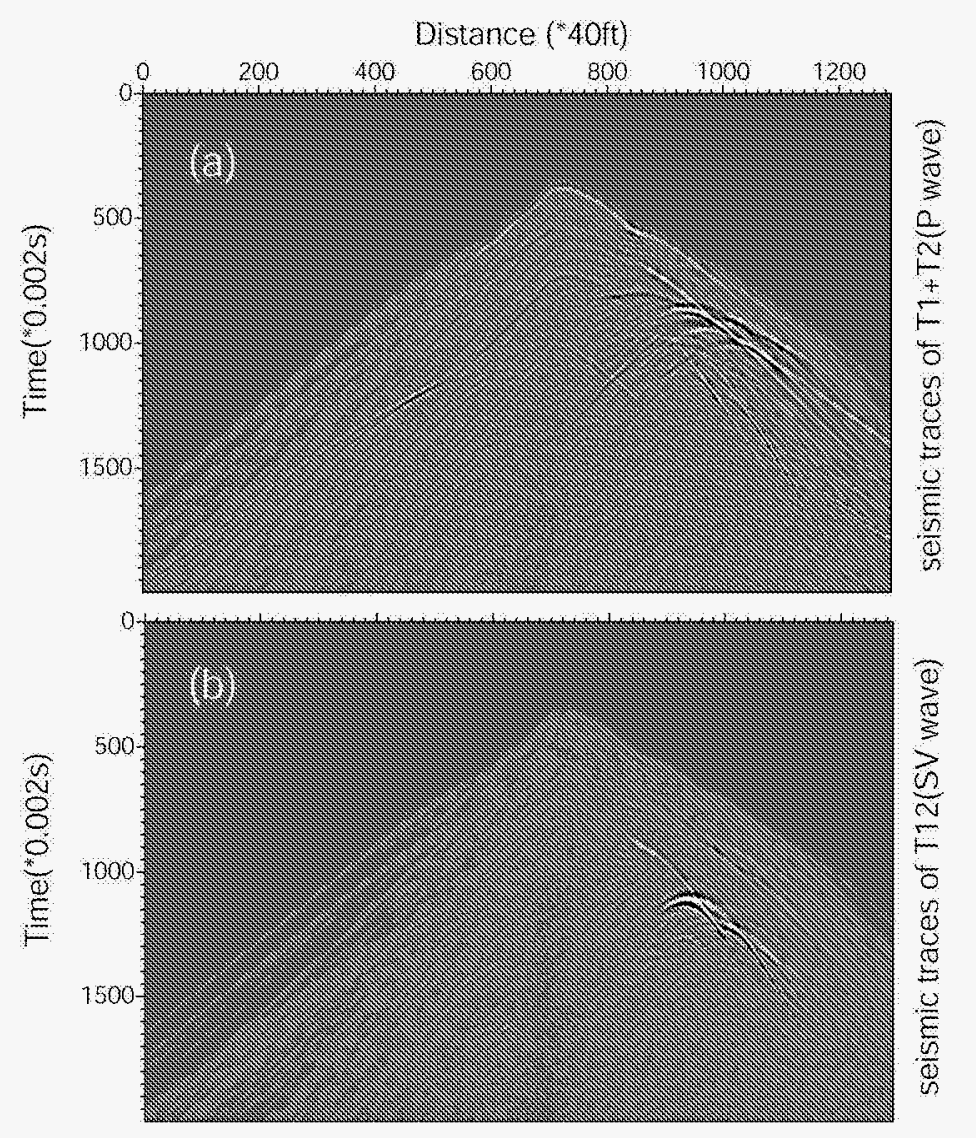

Figure 5. P-wave (a) and S-wave (b) seismograms when exploding reflectors are located at sub-salt fault B.

\subsection{Forward Modeling for Illumination of Subsalt Reflectors}

First, forward modeling is done with exploding reflectors located at different sub-salt faults. The algorithm is a $2 \mathrm{D}$ elastic finite difference code for full elastic wave modeling. These simulations with exploding reflectors on the faults are similar to the case of zero offset experiments. The reflections for the sub-salt faults correspond to the case of planewave normal incidence and normal P-P refection. From these modeling we can see the propagation and conversion of $\mathrm{P}$ waves and $\mathrm{SV}$ waves, and the waves which could finally reach the surface. Secondly, the seismograms we obtained in the forward modeling are used later for the imaging tests.

The first example is for sub-salt fault B (second sub-salt fault from the left) which has a dip angle of about 45 degrees. From the snapshots in Figure 4, we can see clearly a strong $\mathrm{SV}$ wave is formed

at the bottom of the salt body. The converted $\mathrm{S}$ wave can penetrate the salt body and reach the surface as either $\mathrm{P}$ wave (converted again at the salt top) or as $\mathrm{S}$ wave (transmitted through the salt top). On the other hand, only weak P waves from the tip of the fault can propagate through the salt body. The salt $\mathrm{C}$-waves finally reache the surface with remarkable strength (see seismograms in Figure 5). In the elastic finite difference 
algorithm, we have options to output either $\mathrm{P}$ wave only or $\mathrm{S}$ wave only, which are shown in Figure 5a and 5b, respectively. For $\mathrm{S}$ waves, we plot the magnitude of the vector field and treat the $\mathrm{S}$-wave as a quasi-scalar wave.
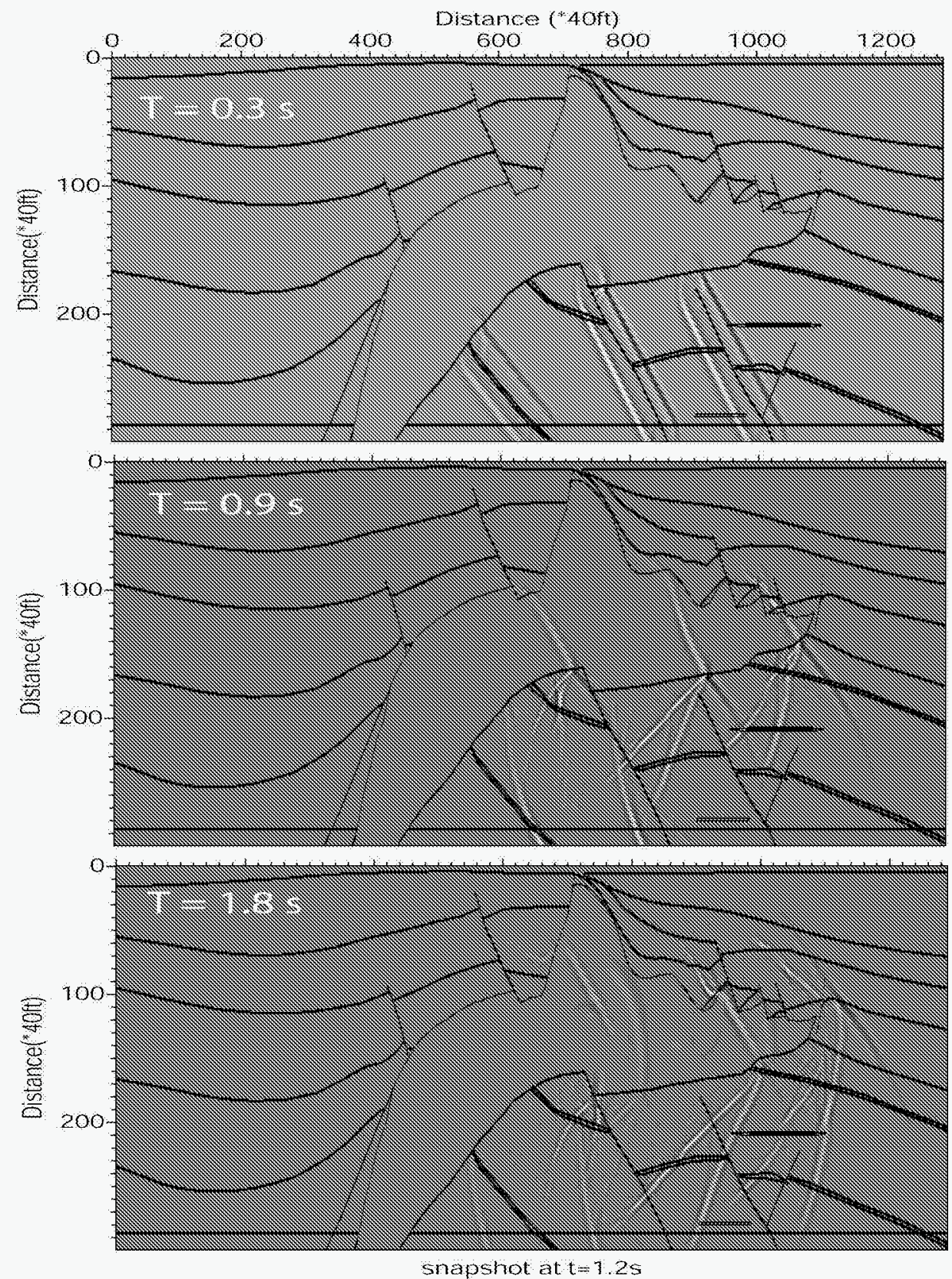

Figure 6. Snapshots when exploding reflectors are located at all three steep sub-salt faults $\mathrm{A}, \mathrm{B}$ and $\mathrm{C}$. 

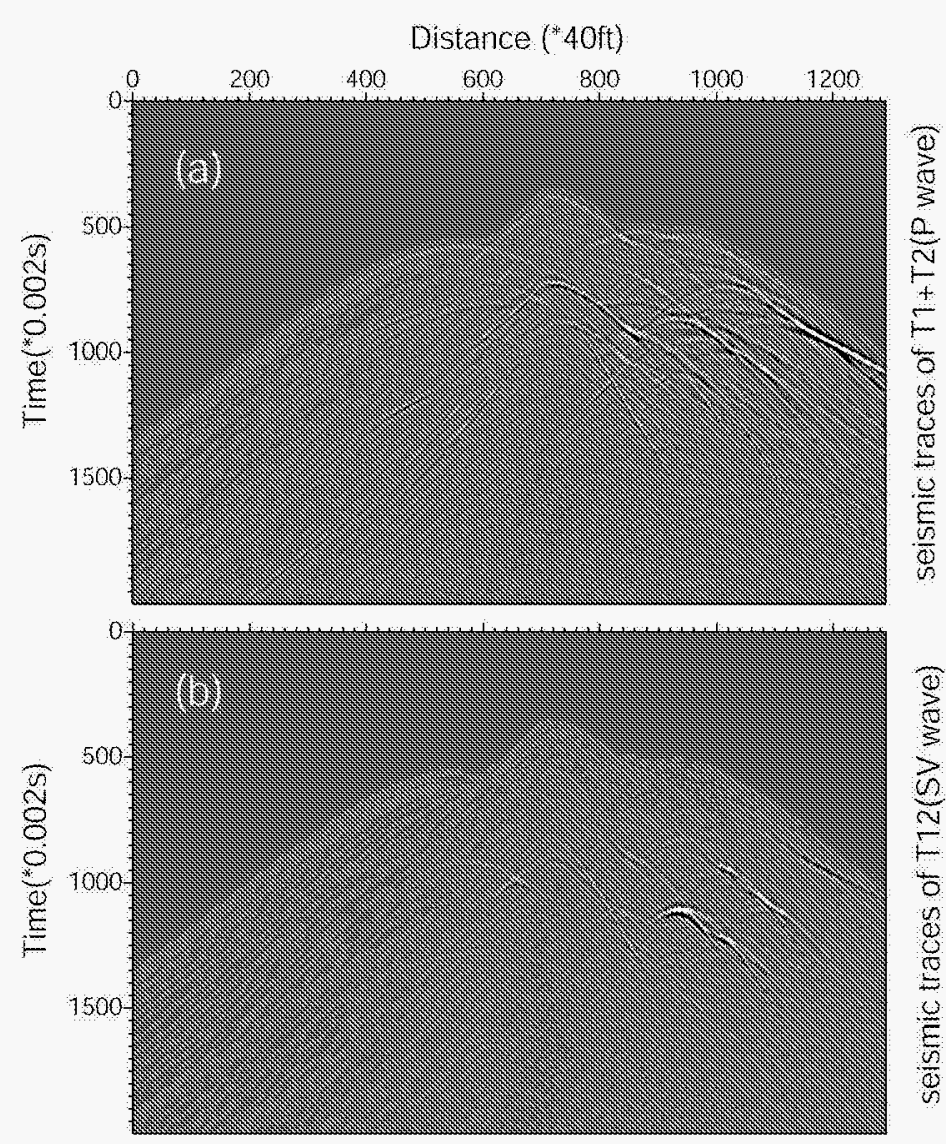

Figure 7. P-wave (a) and S-wave (b) seismograms when exploding reflectors are located at all three steep faults $\mathrm{A}, \mathrm{B}$ anc $\mathrm{C}$.

Putting exploding reflectors at all the three steep sub-salt faults, the propagation and conversion of waves can be seen from the snapshots in Figure 6. The corresponding $\mathrm{P}$ and $\mathrm{S}$ seismograms are shown in Figure $7 \mathrm{a}$ and $7 \mathrm{~b}$, respectively.

From both the snapshots and seismograms, we see strong $S$ waves converted at the salt bottom and reached the surface of the model. However, since the slopes of the faults and the traveling paths of the $\mathrm{P}$ waves are different, the strengths of the $\mathrm{P}$ waves recorded on the surface are different

for the three faults. $\mathrm{P}$ waves from the right two faults ( $\mathrm{B}$ and $\mathrm{C}$ ) are almost all reflected back

at the lower boundary of the salt dome. However, part of the $\mathrm{P}$ wave from the left-most fault (A) went through the salt dome due to the short oblique edge of the salt bottom.

\subsection{Acoustic Migration with Converted Wave Paths}

From the $\mathrm{P}$ wave and $\mathrm{S}$ wave seismograms recorded on the surface (land survey or OBS survey), we can migrate the data using different converted wave paths to improve the imaging for sub-salt structures, especially for steep sub-salt structures. The acoustic migration algorithms can be still used with different velocity models for different C-paths. 
The problem is the relative strength of the C-wave events. From Figure 3, we see that converted arrivals (PSP or PSS) are weak events compared with the P wave arrival (PPP) from the flat (nearly horizontal) reflectors. The transmission efficiency of the C-paths (PSP or PSS) is about 0.175 , i.e. one fifth to one sixth of the P-path efficiency. This is about the amplitude ratio of recorded C-path events (PSPPSP or PSPPSS) to the P-path event (PPPPPP) considering the relation between energy ratio and amplitude ratio, and the two-way transmission losses. Therefore, if we use the P-data for C-path migration (path PSPPSP), the migration noises from the flat sub-salt structures could obscure the images of the steep faults migrated from the PSPPSP paths. Hanssen and Li (2000) noticed the weakness of the C-wave events and pointed out the difficulty in utilizing these events for sub-basalt imaging. On the other hand, S-data can be separated from the P-data. S-data have also much less signals from the flat sub-salt faults because of the unfavorable conversion condition at the salt bottom. In view of this, the S-data will have advantage for steep sub-salt imaging using C-paths.

In the following we will show the example of sub-salt imaging for the SEG-EAGE model. To simplify the demonstration. we test the subsalt imaging by poststack migration using synthetic data of the SEG-EAGE salt model, generated by exploding reflector modeling with our elastic finite difference algorithm. Because of the substantial difference between the strengths of conventional P-path imaging and the C-path imaging, we implement the P-path imaging using the P-data and the C-path imaging using S-data separately. In this way, the weak steep sub-salt images will not be buried into the noise of distorted images of flat sub-salt structures.

Figure $8 \mathrm{a}$ is the migrated image using the hybrid pseudo-screen propagator algorithm (Jin et al., 1998, 1999) with the conventional P-velocity model. The wave paths in this imaging are all $\mathrm{P}$-wave paths. We see that the near horizontal sub-salt structures are well imaged because of the favorable P-wave illumination condition. In contrast, most of the steep faults are missing. These steep faults are nearly 45 degrees and are in the shadow zone of P-path illumination. Figure $8 \mathrm{~b}$ is the migrated image with the C-path (PSS). The velocity below the salt is set as $\mathrm{P}$ velocity; while the velocity above the lower boundary of the salt body is replaced with $\mathrm{S}$ velocity. There is some ambiguity in setting the velocity in the upper-right corner outside the salt boundary. We take the natural extension of the salt bottom to the right as the border line for the separation of $\mathrm{P}$ and $\mathrm{S}$ velocities. We see that the steep sub-salt faults are well imaged, though some noises and artifacts are generated by the edge effects of the salt body. Figure $8 \mathrm{c}$ is summation of the P-path image and C-path image after proper normalization. By separating the P-wave data and $\mathrm{S}$-wave data, and imaging using P-path and C-path separately, all the sub-salt structures, including the steep sub-salt faults can be clearly identified. 

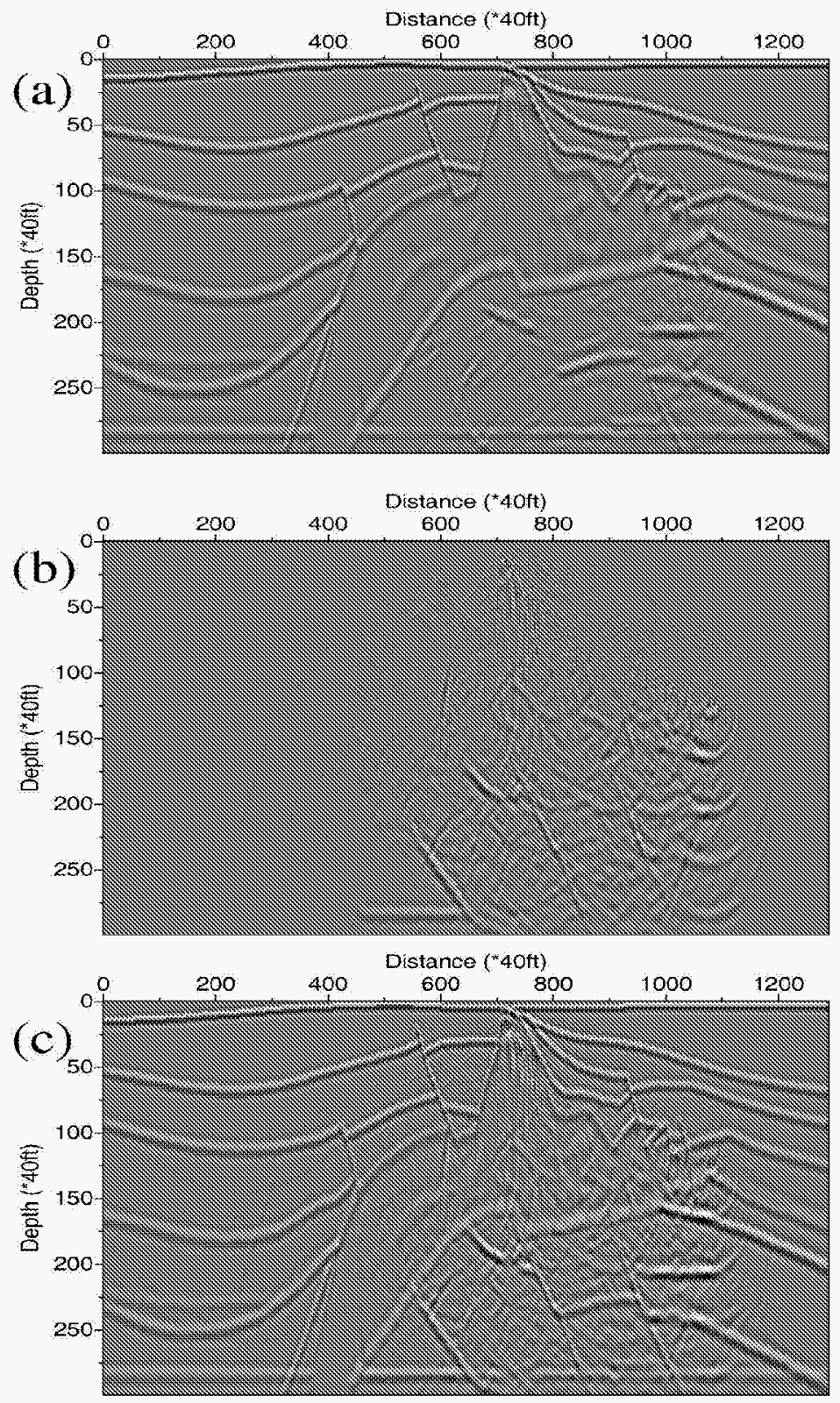

Figure 8. The poststack images for the 2D SEG/EAGE salt model using the GSP method and different wave paths. (a) is the image with P-path using P-data; (b) the image with converted path (C-path) using S-data; (c) the summation of the two images after normalization. Both flat and steep subsalt structures can be seen clearly. 


\section{REFERENCES}

Aki, K., and Richards, P.G., 1980, Quantitative seismology, Theory and methods, Volume I: Freeman and Company.

Alkhalifah, T., 1995, Efficient synthetic-seismogram generation in transversely isotropic, inhomogeneous media: Geophysics, 60, 1139-1150.

Alkhalifah, T., 1998, Acoustic approximations for processing in transversely isotropic media: Geophysics, 63, no. 02, 623-631.

Aminzadeh, F., Brac, J., and Kunz, T., 1997, SEG/EAGE 3-D salt and overthrust models, in SEG/EAGE 3-D Modeling Series, No. 1: Society of Exploration Geophysicists.

Amundsen, L., and Reitar, A., 1995, Extraction of P- and S-waves from the vertical component of the particle velocity at the sea floor: Geophysics, 60, 231-240.

Bear, G., Lu, C., Lu, R. and Willen, D., 2000, The construction of subsurface illumination and amplitude maps via ray tracing, The Leading Edge, 19(7), 726-728.

Berkhout, A.J., 1997, Pushing the limits of seismic imaging, Part I: Prestack migration in terms of double dynamic focusing: Geophysics, 62, 947-954.

Biondi, B. 2002, Stable wide-angle Fourier finite-difference downward extrapolation of 3-D wavefields: Geophysics, 67, 872-882.

Cerveny, V., 1972, Seismic rays and ray intensities in inhomogeneous anisotropic media: Geophys. J. R. astr. Soc., 29, 1-13.

Chang, W.F., and McMechan, G.A., 1987, Elastic reverse-time migration: Geophysics, 52, 1367-1375.

Chang, W.F., and McMechan, G.A., 1994, 3-D elastic prestack reverse-time depth migration: Geophysics, 59, 597-609.

Dai, T.F., and Kuo, J.T., 1986, Real data results of Kirchhoff elastic wave migration: Geophysics, 51, 1006-1011.

Daley, P. F., and Hron, F., 1977, Reflection and transmission coefficients for transversely isotropic media: Geophysics, 67, 661-675.

Fehler, M., H. Sato, and L.-J. Huang, 2000, Envelope broadening of outgoing waves in 2D random media: a comparison between the Markov approximation and numerical simulations, Bull. Seismol. Soc. Am, 90, 914-928.

Ferguson, R. J., and Margrave, G. F., 2002, Depth imaging in anisotropic media by symmetric non-stationary phase shift: Geophys. Prosp., 50, 281-288.

Fisk, M.D., and McCartor, G.D., 1991, The phase screen method for vector elastic waves: Journal of Geophysical Research, 96, 5985-6010.

Fowler, C.M.R., 1990, The solid earth, An introduction to global geophysics: Cambridge University Press.

French, W.S., 1974, Two-dimensional and three-dimensional migration of modelexperiment reflection profiles, Geophysics, 39,265-277.

Gardner, G.H.F., Gardner, L.W. and Gregory, A.R., 1974, Formation velocity and density-the diagnostic basics for stratigraphic traps: Geophysics, 39, 770-780.

Gonzalez, A., Lynn, W., and Robinson, W. F., 1991, Prestack frequency-wavenumber f-k migration in a transversely isotropic medium: 61st Annual Internat. Mtg., Soc. Expl. Geophys., Expanded Abstracts, 1155-1157. 
Han, Q. and R.S. Wu, 2003, One-way dual-domain propagators for scalar P-wave in VTI media,73rd Annual Internat. Mtg., Soc. Expl. Geophys., Expanded Abstracts, 157-160.

Han, Q. and R.S. Wu, 2005, A one-way dual-domain propagator for scalar qP-waves in VTI media, Geophysics, in press.

Hanssen, P., and Li, X.Y, 2000, Converted waves for sub-basalt imaging?, Expanded Abstracts of the Technical Program, SEG 70th Annual Meeting, 1174-1177.

Hoffmann, J., 2001, Illumination, resolution, and image quality of PP- and PS-waves for survey planning, The Leading Edge, 20(9), 1008-1014.

Hokstad, K., 2000, Multicomponent Kirchhoff migration: Geophysics, 65, 861-873.

Hou, A., and Marfurt, K.J., 2002, Multicomponent prestack depth migration by scalar wavefield extrapolation, Geophysics, 67, 1886-1894.

Huang, L.J., Fehler, M.C., and Wu, R.S., 1999, Extended local Born Fourier migration method: Geophysics, 64, 1524-1534.

Huang, L.J., and Fehler, M.C., 2000, Globally optimized Fourier finite-difference migration method: 70th Annual International Meeting, SEG, Expanded Abstracts, $802-805$.

Isaac, J. H., and Lawton, D. C., 1999, Image mispositioning due to dipping TI media: a physical seismic modeling study: Soc. Expl. Geophys.

Jin, S., Wu, R.S., and Peng, C., 1998a, Prestack depth migration using a hybrid pseudoscreen propagator: 68th Annual International Meeting, SEG, Expanded Abstracts, 1819-1822.

Jin, S., Wu, R.S., and Peng, C., 1999, Seismic depth migration with pseudo-screen propagators: Computational Geosciences, 3, 321-335.

Jin, S., Wu, R.S., Xie, X.B., and Ma, Z., 1998b, Wave equation-based decomposition and imaging for multicomponent seismic data: Journal of Seismic Exploration, 7, 145-158.

Jin, S., and Wu, R. S., 1999, Common offset pseudo-screen depth migration, Annual Internat. Mtg., Soc. Expl. Geophys., Expanded Abstracts, 1516-1519.

Jin, S., Mosher, C.C., and Wu, R.S., 2000, 3D prestack wave equation common offset pseudo-screen depth migration, Annual Internat. Mtg., Soc. Expl. Geophys, 842-845.

Jin, S., Mosher, C.C., and Wu, R.S., 2002, Offset-domain pseudoscreen prestack depth migration: Geophysics, 67, 1895-1902.

Jones, N. and Gaiser, J., 1999, Imaging beneath high-velocity layers, Expanded Abstracts of the Technical Program, SEG 69th Annual Meeting, 713-716.

Kendall, R.R., Cray, S.H. and Murphy, G.E., 1998, Subsalt imaging using prestack depth migration of converted waves: Mahogany Field, Gulf of Mexico, Expanded Abstracts of the Technical Program, SEG 68th Annual Meeting, 2052-2055.

Kitchenside, P. W., 1991, Phase-shift based migration for transverse isotropy: $61^{\text {st }}$ Annual Internat. Mtg., Soc. Expl. Geophys., Expanded Abstracts, 993-996.

Krey, T. H., and Helbig, K., 1956, A theorem concerning anisotropy of stratified media and its significance for reflection seismics: Geophys. Prosp., 4, 294-302.

Kuo, J.T., and Dai, T.F., 1984, Kirchhoff elastic wave migration for the case of noncoincident source and receiver: Geophysics, 49, 1223-1238.

Larner, K., and Cohen, J. K., 1993, Migration error in transversely isotropic media with linear velocity variation in depth: Geophysics, $\mathbf{5 8}$, no. 10, 1454-1467. 
Le Rousseau, J., and de Hoop, M., 2001, Scalar generalized-screen algorithms in transversely isotropic media with a vertical symmetry axis: Geophysics, 66, 5381550.

Le Rousseau, J. H., 1997, Depth migration in heterogeneous, transversely isotropic media with the phase-shift-plus-interpolation method: 67th Ann. Internat. Mtg., Soc. Expl. Geophys., 1703-1706.

Li, X.Y., MacBeth, C., Hitchen, K. and Hanssen, P., 1998, Using converted shear-waves for imaging beneath basalt in deep water plays, Expanded Abstracts of the Technical Program, SEG 68th Annual Meeting, 1369-1372.

Liu, W., Popovici, A.M., Bevc, and D., Biondi, B., 2001, 3D migration velocity analysis for common image gathers in the reflection angle domain, Annual Internat. Mtg., Soc. Expl. Geophys., Expanded Abstracts, 885-888.

Luo, M. and Wu, R.S., 2003, 3D beamlet prestack depth migration using the local cosine basis propagator, submitted to the SEG 73rd Annual Meeting.

Martin, D., Ehinger, A., and Rasolofosaon, P. N. J., 1992, Some aspects of seismic modeling and imaging in anisotropic media using laser ultrasonics: 62nd Annual Internat. Mtg., Soc. Expl. Geophys., Expanded Abstracts, 1373-1376.

Meadows, M., and Abriel, W. L., 1994, 3-d phase-shift migration in transversely isotropic media: 64th Ann. Internat. Mtg., Soc. Expl. Geophys., 1331-1348.

Meadows, M., Coen, S., and Liu, G., 1987, F-K migration in elliptically anisotropic media: 57th Ann. Internat. Mtg., Soc. Expl. Geophys., 659-661.

Mittet, R., Sollie, R., and Hokstad, K., 1995, Prestack depth migration with compensation for absorption and dispersion: Geophysics, 60, 1485-1494.

Mosher, C., 2001, Migration velocity analysis using common angle image gathers, Annual Internat. Mtg., Soc. Expl. Geophys., Expanded Abstracts, 889-892.

Mosher, C.C. and Foster, D.J., 1998, Offset plane wave propagation in laterally varying media,, in Mathematical Methods in Geophysical Imaging V, S. Hassanzadeh, ed., Proc. SPIE 3453, 36-46.

Mosher, C.C., Foster, D.J., and Hassanzadeh, S., 1996, Seismic imaging with offset plane waves, in Mathematical Methods in Geophysical Imaging IV, S. Hassanzadeh, ed., Proc. SPIE 2822, 52-63.

Muerdter, D. and Ratcliff, D., 2001a, Understanding subsalt illumination through raytrace modeling, Part 1: Simple 2-D salt models, The Leading Edge, 20(6), 578-594.

Muerdter, D., Kelly, M. and Ratcliff, D., 2001b, Understanding subsalt illumination through ray-trace modeling, Part 2: Dipping salt bodies, salt peaks, and nonreciprocity of subsalt amplitude response, The Leading Edge, 20(7), 688-697.

Muerdter, D. and Ratcliff, D., 2001c, Understanding subsalt illumination through raytrace modeling, Part 3: Salt ridges and furrows, and the impact of acquisition orientation, The Leading Edge, 20(8), 803-816.

O'Brien, M.J., and Gray, S.H., 1996, Can we image beneath salt: The Leading Edge, 15, 17-22.

O'Brien, M.J., Whitmore, N.D., Murphy, G.E. and Etgen, J.T., 1999, Multicomponent imaging with reciprocal shot records, Expanded Abstracts of the Technical Program, SEG 69th Annual Meeting,784-787. 
Pearce, F., D. Burns, R. Rao, M. Willis, and J. Byun, 2003, Fracture Density Estimation Using Spectral Analysis of Reservoir Reflections: A Numerical Modeling Approach, Earth Resources Laboratory, Industry consortia, Annual Report.

Pride, S, J. Harris, D. Johnson, A. Mateeva, K. Nihei, R. Nowack, J. Rector, H. Spetzler, R. Wu, T. Yamomoto, J. Berryman, and M. Fehler, 2003, Permeability dependence of seismic Amplitudes, The Leading Edge, 518-525.

Prucha, M., Biondi, B. and Symes, W., 1999, Angle-domain common image gathers by wave-equation migration: Annual Internat. Mtg., Soc. Expl. Geophys., Expanded Abstracts, 824-827.

Purnell, G.W., 1992, Imaging beneath a high-velocity layer using converted waves, Geophysics, 57, 1444-1452.

Rickett, J. and Sava, P., 2001, Offset and angle domain common-image gathers for shotprofile migration, Annual Internat. Mtg., Soc. Expl. Geophys., Expanded Abstracts, 1115-1118.

Ristow, D., and Ruhl, T., 1994, Fourier finite-difference migration: Geophysics, 59, 1882-1893.

Ristow, D., and Ruhl, T., 1997, Migration in transversely isotropic media using implicity operators: 67th Ann. Internat. Mtg., Soc. Expl. Geophys., 1699-1702.

Saito, T., H. Sato, M. Fehler, and M. Ohtake, 2003, Simulating the envelope of scalar waves in 2-D random media having power-law spectra of velocity fluctuation, Bull. Seismol Soc. Am. 93, 240-252.

Sato, H. and M. Fehler, 1998, Seismic Wave Propagation and Scattering in the Heterogeneous Earth, Springer-Verlag \& American Institute of Physics Press.

Sato, H., M. Fehler, and T. Saito, 2003, Hybrid Synthesis of Scalar Wave Envelopes in 2D Random Media Having Rich Short Wavelength Spectra, submitted.

Sato, H., M. Fehler, and R.S. Wu, 2002, Scattering and attenuation of seismic waves in the lithosphere (invited book chapter), International Handbook of Earthquake and Engineering Seismology, (Eds. P. Jennings, H. Kanamori, and W. Lee), 195-208, Academic Press.

Schneider, W.A. and Winbow, G.A., 1999, Efficient and accurate modeling of 3-D seismic illumination, 69th Ann. Internat. Mtg., Soc. Expl. Geophys., Expanded Abstracts, 633-636.

Sheriff, R.E., 1991, Encyclopedic dictionary of exploration geophysics, Third edition: Society of Exploration Geophysicists.

Schoenberg, M. A., and de Hoop, M., 2000, Approximate dispersion relations for qPqSV-waves in transversely isotropic media: Geophysics, 65, no. 03, 919-933.

Sena, A. G., and Toksoz, M. N., 1993, Kirchhoff migration and velocity analysis for converted and nonconverted waves in anisotropic media: Geophysics, 58, no. 02, 265-276.

Stoffa, P.L., Fokkema, J.T., Freire, R.M.D., and Kessinger, W.P., 1990, Split-step Fourier migration: Geophysics, 55, 410-421.

Sun, R., Chow, J., and Chen, K.J., 2001a, Phase correction in separating P- and S-waves in elastic data: Geophysics, 66, 1515-1518.

Sun, R., and McMechan, G.A., 2001b, Scalar reverse-time depth migration of prestack elastic seismic data: Geophysics, 66, 1519-1527. 
Thomsen, L., 1986, Weak elastic anisotropy: Geophysics, 51, no. 10, 1954-1966.

Thomsen, L., 1998, Converted-wave reflection seismology over anisotropic, inhomogeneous media, Expanded Abstracts of the Technical Program, SEG 68th Annual Meeting, 2048-2051.

Sun, R., Chow, J., and Chen, K.J., 2001, Phase correction in separating $P$ - and $S$-waves in elastic data: Geophysics, 66, 1515-1518.

Sun, R., and McMechan, G.A., 2001, Scalar reverse-time depth migration of prestack elastic seismic data: Geophysics, 66, 1519-1527.

Uren, N. F., Gardner, G. H. F., and McDonald, J. A., 1990, The migrator's equation for anisotropic media: Geophysics, 55, no. 11, 1429-1434.

Uzcategui, O., 1995, 2-D depth migration in transversely isotropic media using explicit operators: Geophysics, 60, no. 06, 1819-1829.

Wild, A.J., and Hudson, J.A., 1998, A geometrical approach to the elastic complex screen: Journal of Geophysical Research, 103, 707-725.

Wu, R.S., 1994, Wide-angle elastic wave one-way propagation in heterogeneous media and an elastic wave complex-screen method: Journal of Geophysical Research, 99, 751-766.

Wu, R.S., 1996, Synthetic seismograms in heterogeneous media by one-return approximation: Pure and Applied Geophysics, 148, 155-173.

$\mathrm{Wu}, \mathrm{R}$. S., 2003, Wave propagation, scattering and imaging using dual-domain one-way and one-return propagators: Pure and Appl. geophys., 160 (3/4), 509-539.

$\mathrm{Wu}, \mathrm{R} . \mathrm{S}$., and Aki, K., 1985, Scattering characteristics by an elastic heterogeneity, Geophysics, 50, 582-595.

Wu, R.S. and Chen, L., 2001, Beamlet migration using Gabor-Daubechies frame propagator, 63rd, Conference and Technical Exhibition, EAGE, Expanded Abstracts.

$\mathrm{Wu}, \mathrm{R} . \mathrm{S}$. and Chen, L, 2002, Mapping directional illumination and acquisition-aperture efficacy by beamlet propagators, Annual Internat. Mtg., Soc. Expl. Geophys., Expanded Abstracts, 1352-1355.

Wu, R.S. and L. Chen, 2003, Prestack depth migration in angle-domain using beamlet decomposition: Local image matrix and local AVA, 73rd Annual Internat. Mtg., Soc. Expl. Geophys., Expanded Abstracts, 973-976.

Wu, R.S., Guan, H. and Wu X.Y., 2001, Imaging steep sub-salt structures using converted wave paths, Expanded abstracts, SEG 71st Annual Meeting, 845-848.

Wu, R.S., Wang, Y. and Gao, J.H., 2000, Beamlet migration based on local perturbation theory, Annual Internat. Mtg., Soc. Expl. Geophys., Expanded Abstracts, 1008-1011.

Wu, R.S., and Xie, X.B., 1994, Multi-screen back propagator for fast 3D elastic prestack migration: Mathematical Methods in Geophysical Imaging II, SPIE, 2301, 181-193.

Wu, X.Y. and R.S. Wu, 1999, Wide-angle thin-slab propagator with phase matching for elastic wave modeling, Expanded Abstracts, SEG 69th Annual Meeting, 1867-1870.

$\mathrm{Wu}, \mathrm{X} . \mathrm{Y}$. and R.S. Wu, 2001, AVO modeling using one-return approximation, 71st Annual Internat. Mtg., Soc. Expl. Geophys., Expanded Abstracts, 312-315.

$\mathrm{Wu}, \mathrm{X} . \mathrm{Y}$, and R.S. Wu, 2003a, Fast modeling of 2D/3D elastic reflections using thin-slab method, 73rd Annual Internat. Mtg., Soc. Expl. Geophys., Expanded Abstracts, 1865 1868. 
$\mathrm{Wu}, \mathrm{X} . \mathrm{Y}$, and R.S. Wu, 2003b, Synthesizing AVO responses in visco-elastic media using fast one-way elastic propagators, 73rd Annual Internat. Mtg., Soc. Expl. Geophys., Expanded Abstracts, 208-210.

Wu, X.Y., and R.S. Wu, 2004, AVO modeling using elastic thin-slab method, Geophysics, accepted.

Xie, X.B., and Lay, T., 1994, The excitation of Lg waves by explosions: A finitedifference investigation: Bulletin of the Seismological Society of America, 84, 324342.

Xie, X.B., and Wu, R.S., 1996, 3D elastic wave modeling using the complex screen method: 66th Annual International Meeting, SEG, Expanded Abstracts, 1247-1250.

Xie, X.B., and Wu, R.S., 1998, Improve the wide angle accuracy of screen method under large contrast: 68th Annual International Meeting, SEG, Expanded Abstracts, 12471250.

Xie, X.B., and Wu, R.S., 1999, Improve the wide angle accuracy of the screen propagator for elastic wave propagation: 69th Annual International Meeting, SEG, Expanded Abstracts, 1863-1866.

Xie, X.B., Mosher, C.C., and Wu, R.S., 2000, The application of wide angle screen propagator to 2D and 3D depth migrations: 70th Annual International Meeting, SEG, Expanded Abstracts, 878-881.

Xie, X.B., and Wu, R.S., 2001, Modeling elastic wave forward propagation and reflection using the complex screen method: Journal of the Acoustic Society of America, 109, 2629-2635.

Xie, X.B. and Wu, R.S., 2002, Extracting angle domain information from migrated wavefield, Expanded Abstracts, SEG 72th Annual Meeting, 1360-1363.

Xie, X.B., S. Jin, and R.S. Wu, 2003, Three-dimensional illumination analysis using wave equation based propagator, 73rd Annual Internat. Mtg., Soc. Expl. Geophys., Expanded Abstracts, 989-992.

Xie, X.B. and R.S. Wu, 2005, Multicomponent elastic wave prestack depth migration using elastic screen method, Geophysics, 70, S30-37.

Zhe, J., and Greenhalgh, S.A., 1997, Prestack multi-component migration: Geophysics, 62, 598-613. 


\section{RELATED PUBLICATIONS}

Chen, L. and R.S. Wu, 2002, Target-oriented prestack beamlet migration using GaborDaubechies frames, 72nd Annual Internat. Mtg., Soc. Expl. Geophys., Expanded Abstracts, 1356-1359.

Chen, L., R.S. Wu, Y. Chen, and W. Wang, 2001, Acoustic wavelets applied to seismic signal decomposition, Chinese Journal of Geophysics, 44, 369-378.

Chen, S. and R.S. Wu, 2003, Estimating the effects of number of shots and propagator apertures to prestack depth migration through the mapping of acquisition dipresponse, 73rd Annual Internat. Mtg., Soc. Expl. Geophys., Expanded Abstracts, 1017-1019.

Chen, L., R.S. Wu, and Y. Chen, 2004, Applications of prestack beamlet migration using Gabor-Daubechies frames, CNPC/SEG Beijing Conference.

De Hoop, M., J. Le Rousseau, and R.S. Wu, 2000, Generalization of the phase-screen approximation for the scattering of acoustic waves, Wave Motion, 31, 43-70.

Foster, D.J., R.S. Wu, and C.C. Mosher, 2002, Coherent-state solutions of the wave equation, 72nd Annual Internat. Mtg., Soc. Expl. Geophys., Expanded Abstracts, 1348-1351.

Gao, J.H., R.S. Wu, and B.J. Wang, 2001, A new type of analyzing wavelet and its applications for extraction of instantaneous spectrum bandwidth, 71st Annual Internat. Mtg., Soc. Expl. Geophys., Expanded Abstracts, 1937-1940.

Guan, H., R.S. Wu, and L.Y. Fu, 2000, Removing scattering effects of rugged topography using finite difference method, 70th Annual Internat. Mtg., Soc. Expl. Geophys., Expanded Abstracts, 2189-2192.

Han, Q. and R.S. Wu, 2003, One-way dual-domain propagators for scalar P-wave in VTI media,73rd Annual Internat. Mtg., Soc. Expl. Geophys., Expanded Abstracts, 157-160.

Han, Q. and R.S. Wu, 2005, A one-way dual-domain propagator for scalar qP-waves in VTI media, Geophysics, in press.

Jin, S., C.C. Mosher, and R.S. Wu, 2000, 3-D prestack wave equation common offset pseudo-screen depth migration, 70st Annual Internat. Mtg., Soc. Expl. Geophys., Expanded Abstracts, 842-845.

Jin, S., C.C. Mosher, and R.S. Wu, 2002, Offset-domain pseudo-screen prestack depth migration, Geophysics , 67, 1895-1902.

Luo, M. and R.S. Wu, 2003, 3D beamlet prestack depth migration using the local cosine basis propagator, 73rd Annual Internat. Mtg., Soc. Expl. Geophys., Expanded Abstracts, 985-988.

Mosher, C.C., R.S. Wu, and D.J. Foster, 2002, Phase shift migration using orthogonal beamlet transforms, 72nd Annual Internat. Mtg., Soc. Expl. Geophys., Expanded Abstracts,1332-1335.

Wang, Y. and R.S. Wu, 2000, Improvements on seismic data compression and migration using compressed data with the flexible segmentation scheme for local cosine transform, 70th Annual Internat. Mtg., Soc. Expl. Geophys., Expanded Abstracts, 2048-2051.

Wang, Y. and R.S. Wu, 2000, Seismic data Compression by an adaptive local cosine/sine transform and its effects on migration, Geophysical Prospecting, 48, 1009-1031. 
Wang, Y. and R.S. Wu, 2002, Beamlet prestack depth migration using local cosine basis propagator, 72nd Annual Internat. Mtg., Soc. Expl. Geophys., Expanded Abstracts, 1340-1343.

Wang, Y., R. Cook, and R.S. Wu, 2003, 3D local cosine beamlet propagator, 73rd Annual Internat. Mtg., Soc. Expl. Geophys., Expanded Abstracts, 981-984.

Wang, Y., W.B. Wang, and R.S. Wu, 2000, Attenuation and dispersion of a monopulse in layered and lossy media using an adaptive multiscale wavelet collocation method, Wavelet Applications in Signal and Image Processing VIII, Proceedings of SPIE, 4119, 983-990.

Wu, R.S., Guan, H. and Wu X.Y., 2001, Imaging steep sub-salt structures using converted wave paths, Expanded abstracts, SEG 71st Annual Meeting, 845-848.

Wu, R.S. and L. Chen, 2001, Beamlet migration using Gabor-Daubechies frame propagator, 63rd Conference \& Technical Exhibition, EAGE, Expanded abstracts, 74.

$\mathrm{Wu}$, R.S. and L. Chen, 2002, Mapping directional illumination and acquisition-aperture efficacy by beamlet propagators, 72nd Annual Internat. Mtg., Soc. Expl. Geophys., Expanded Abstracts, 1352-1355.

Wu, R.S. and L. Chen, 2003, Prestack depth migration in angle-domain using beamlet decomposition: Local image matrix and local AVA, 73rd Annual Internat. Mtg., Soc. Expl. Geophys., Expanded Abstracts, 973-976.

Wu, R.S., Guan, H. and Wu X.Y., 2001, Imaging steep sub-salt structures using converted wave paths, Expanded abstracts, SEG 71st Annual Meeting, 845-848.

$\mathrm{Wu}, \mathrm{R} . \mathrm{S} ., 2003$, Wave propagation, scattering and imaging using dual-domain one-way and one-return propagators, Pure and Appl. Geophys. (Special Issue), 160(3/4), 509539.

Wu, R.S., L. Chen, and X.B. Xie, 2003, Directional illumination and acquisition dipresponse, 65th Conference \& Technical Exhibition, EAGE, Expanded abstracts, P147.

Wu, R.S., L. Chen, and Y. Wang, 2002, Synthetic beam-sources and plane-sources for prestack beamlet migration, 72nd Annual Internat. Mtg., Soc. Expl. Geophys., Expanded Abstracts, 1336-1339.

Wu, R.S., S. Jin, and X.B. Xie, 2000, Energy partition and attenuation of Lg waves by numerical simulations using screen propagators, Phys, Earth Planet. Inter., 120, 227244.

Wu, R.S., S. Jin, and X.B. Xie, 2000, Seismic wave propagation and scattering in heterogeneous crustal waveguides using screen propagators: I SH waves, Bull. Seism. Soc. Am., 90, 401-413.

Wu, R.S., S. Jin, and X.B. Xie, 2001, Generalized screen propagators and the applications to seismic imaging, Oil Geophysical Prospecting , 36, 655-664 (in Chinese with English abstract).

Wu, R.S., S. Jin, X.B. Xie, and C.C. Mosher, 2000, 2D and 3D generalized screen migrations, 62nd Conference \& Technical Exhibition, EAGE, Expanded abstracts, B15.

Wu, R.S., Y. Wang, and J. Gao, 2000, Beamlet migration based on local perturbation theory, Expanded abstracts, 70th Annual Internat. Mtg., Soc. Expl. Geophys., Expanded Abstracts, 1008-1011. 
Wu, X.Y, and R.S. Wu, 2003, Fast modeling of 2D/3D elastic reflections using thin-slab method, 73rd Annual Internat. Mtg., Soc. Expl. Geophys., Expanded Abstracts, 18651868.

Wu, X.Y, and R.S. Wu, 2003, Synthesizing AVO responses in visco-elastic media using fast one-way elastic propagators, 73rd Annual Internat. Mtg., Soc. Expl. Geophys., Expanded Abstracts, 208-210.

$\mathrm{Wu}, \mathrm{X} . \mathrm{Y}$. and R.S. Wu, 2001, AVO modeling using one-return approximation, $71 s t$ Annual Internat. Mtg., Soc. Expl. Geophys., Expanded Abstracts, 312-315.

$\mathrm{Wu}, \mathrm{X} . Y$. , and R.S. Wu, 2004, AVO modeling using elastic thin-slab method, Geophysics, accepted.

Xie, X.B, C.C. Mosher, and R.S. Wu, 2000, The application of wide angle screen propagator to 2D and 3D depth migrations, 70th Annual Internat. Mtg., Soc. Expl. Geophys., Expanded Abstracts, 878-881.

Xie, X.B., and R.S. Wu, 2001, Migration of multicomponent seismic data using elastic screen method, SEG 71st Annual Internat. Mtg., Soc. Expl. Geophys., Expanded Abstracts, 1069-1072.

Xie, X.B., and R.S. Wu, 2001, Modeling elastic wave forward propagation and reflection using the complex screen method, J. Acoust. Soc. Am., 109, 2629-2635.

Xie, X.B., and R.S. Wu, 2002, Extracting angle related image from migrated wavefield, Expanded Abstracts, 72nd Annual Internat. Mtg., Soc. Expl. Geophys., Expanded Abstracts, 1360-1363.

Xie, X.B., S. Jin, and R.S. Wu, 2001, 3D prestack depth migration using generalized screen propagators, 63rd Conference \& Technical Exhibition, EAGE, Expanded abstracts, P061.

Xie, X.B., S. Jin, and R.S. Wu, 2003, Three-dimensional illumination analysis using wave equation based propagator, 73rd Annual Internat. Mtg., Soc. Expl. Geophys., Expanded Abstracts, 989-992.

Xie, X.B. and R.S. Wu, 2005, Multicomponent elastic wave prestack depth migration using elastic screen method, Geophysics, 70, S30-37. 